\title{
Oscillatory motions for the restricted planar circular three body problem
}

\author{
Marcel Guardia, Pau Martín ${ }^{\dagger}$ and Tere M. Seara ${ }^{\ddagger}$
}

October 8, 2012

\author{
* Department of Mathematics \\ Mathematics Building, University of Maryland \\ College Park, MD 20742-4015, USA \\ † Departament de Matemàtica Aplicada IV \\ Universitat Politècnica de Catalunya \\ Campus Nord, Edifici C3, C. Jordi Girona, 1-3. 08034 Barcelona, Spain \\ ¥ Departament de Matemàtica Aplicada I \\ Universitat Politècnica de Catalunya \\ Diagonal 647, 08028 Barcelona, Spain
}

\begin{abstract}
In this paper we consider the circular restricted three body problem which models the motion of a massless body under the influence of the Newtonian gravitational force caused by two other bodies, the primaries, which move along circular planar Keplerian orbits. In a suitable system of coordinates, this system has two degrees of freedom and the conserved energy is usually called the Jacobi constant. In 1980, J. Llibre and C. Simó LS80b proved the existence of oscillatory motions for the restricted planar circular three body problem, that is, of orbits which leave every bounded region but which return infinitely often to some fixed bounded region. To prove their existence they had to assume the ratio between the masses of the two primaries to be exponentially small with respect to the Jacobi constant. In the present work, we generalize their result proving the existence of oscillatory motions for any value of the mass ratio.

To obtain such motions, we show that, for any value of the mass ratio and for big values of the Jacobi constant, there exist transversal intersections between the stable and unstable manifolds of infinity which guarantee the existence of a symbolic dynamics that creates the oscillatory orbits. The main achievement is to rigorously prove the existence of these orbits without assuming the mass ratio small since then this transversality can not be checked by means of classical perturbation theory respect to the mass ratio. Since our method is valid for all values of mass ratio, we are able to detect a curve in the parameter space, formed by the mass ratio and the Jacobi constant, where cubic homoclinic tangencies between the invariant manifolds of infinity appear.
\end{abstract}

\footnotetext{
*marcel.guardia@upc.edu, mguardia@umd.edu

${ }^{\dagger}$ martin@ma4.upc. edu

${ }^{\ddagger}$ tere.m-seara@upc. edu
} 


\section{Mouvements oscillatoires dans le problème plan circulaire restreint des trois corps}

Résumé : Dans cet article, nous étudions le problème restreint des trois corps, qui modélise le mouvement d'un corps de masse nulle sous l'influence des forces de gravitation newtonienne créées par deux autres corps, appelés les primaires, qui eux se déplacent le long d'orbites képlériennes circulaires. Dans un système de coordonnées convenable, ce système possède deux degrés de liberté et l'énergie conservée est habituellement appelée la constante de Jacobi. En 1980, J. Llibre et C. Simó [LS80b ont démontré l'existence de mouvements oscillatoires dans le problème plan restreint des trois corps, c'est-àdire d'orbites qui sortent de n'importe quelle région bornée mais qui rentrent une infinité de fois dans une certaine région bornée fixée. Pour démontrer ce résultat, les auteurs avaient besoin de supposer que le rapport des masses des deux primaires est exponentiellement petit par rapport à la constante de Jacobi. Dans le présent travail, nous généralisons ce théorème à toute valeur des rapports de masses. Pour obtenir de tels mouvements, nous montrons que, quel que soit le rapport des masses, si la constante de Jacobi est assez grande, il existe des intersections transverses des variétés stable et instable de l'infini, ce qui garantit l'existence d'une dynamique symbolique, puis celle de mouvements oscillatoires. Le principal résultat est de prouver rigoureusement l'existence de ces orbites sans supposer que le rapport de masses est petit, puisqu'alors la transversalité ne peut pas être vérifiée par les méthodes de la théorie classique des perturbations relativement au rapport de masses. Comme notre méthode est valable pour toutes les valeurs des rapports de masses, nous parvenons à détecter une courbe, dans l'espace des paramétres, c'est-à-dire dans l'espace des rapports de masses et de la constante de Jacobi, sur laquelle apparaissent des tangences homoclines cubiques entre les variétés invariantes de l'infini.

\section{Contents}

1 Introduction 3

2 The invariant manifolds of infinity 6

2.1 The RPC3BP as a nearly integrable Hamiltonian System with two time scales . . . . . . . 7

2.2 Main result: intersection of the invariant manifolds . . . . . . . . . . . . . . . 9

2.3 From transversal homoclinic points to oscillatory motions . . . . . . . . . . . . . . 12

3 Parameterizations of the invariant manifolds as generating functions 13 3.1 Proof of Theorems 2.1 and $2.2 \ldots \ldots \ldots \ldots \ldots \ldots \ldots \ldots$

4 The integrable system and the homoclinic solution 17

5 Existence of the invariant manifolds $\quad 18$

5.1 Description of the proof . . . . . . . . . . . . . . . . . . 19

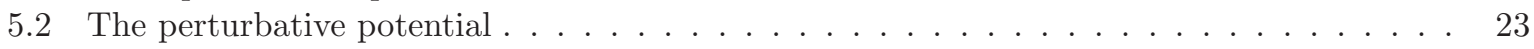

5.3 The invariant manifolds close to infinity . . . . . . . . . . . . . . . . . . . . 24

5.4 The invariant manifolds close to the singularities . . . . . . . . . . . . . . . . . 27

5.4 Weighted Fourier norms and Banach spaces . . . . . . . . . . . . . . . . 28

5.4 The fixed point argument . . . . . . . . . . . . . . . . . . . 29

5.5 Extension of the parameterization of the unstable manifold by the flow . . . . . . . . . . 33

5.5.1 From Hamilton-Jacobi parameterizations to parameterizations invariant by the flow 33

5.5.2 Analytic extension of the unstable manifold by the flow parameterization . . . . . 36

5.5.3 From flow parameterization to Hamilton-Jacobi parameterization . . . . . . . . . 38 
6 The difference between the manifolds 39

6.1 Weighted Fourier norms and Banach spaces . . . . . . . . . . . . . . . . . 40

6.2 Straightening the operator $\widetilde{\mathcal{L}} \ldots \ldots \ldots \ldots \ldots \ldots \ldots \ldots \ldots$

6.3 Estimates for the difference between invariant manifolds . . . . . . . . . . . . . . . . 44

A Computation of the function L: proof of Proposition 3.1

\section{Introduction}

The restricted three body problem models the motion of three bodies, one of them massless, under the Newtonian gravitational force. Since one of the bodies is massless, it does not cause any influence on the other two, the primaries. Thus, the two primaries are governed by the classical Keplerian two body problem. Let us assume that these two bodies, whose motion lies on a plane, perform circular orbits and that the massless body moves in the same plane. This problem is known as the restricted planar circular three body problem (RPC3BP from now on). If one normalizes the total mass of the system to be one, the RPC3BP depends on one parameter $\mu$ which measures the quotient between the masses of the two primaries and therefore satisfies $\mu \in[0,1 / 2]$. Then, taking the appropriate units, the RPC3BP is Hamiltonian with respect to

$$
H(q, p, t ; \mu)=\frac{\|p\|^{2}}{2}-\frac{1-\mu}{\left\|q+\mu q_{0}(t)\right\|}-\frac{\mu}{\left\|q-(1-\mu) q_{0}(t)\right\|}
$$

where $q, p \in \mathbb{R}^{2}$ and $-\mu q_{0}(t)$ and $(1-\mu) q_{0}(t), q_{0}(t)=(\cos t, \sin t)$, are the positions of the primaries. This Hamiltonian has two and a half degrees of freedom. It has a conserved quantity, usually called Jacobi constant, defined as

$$
\mathcal{J}(q, p, t ; \mu)=H(q, p, t ; \mu)-\left(q_{1} p_{2}-q_{2} p_{1}\right)
$$

The purpose of this paper is to study the existence of oscillatory orbits for the RPC3BP, that is, orbits such that

$$
\limsup _{t \rightarrow \pm \infty}\|q\|=+\infty \text { and } \liminf _{t \rightarrow \pm \infty}\|q\|<+\infty .
$$

When $\mu=0$, the motion of the massless body is only influenced by one of the primaries and therefore it satisfies Kepler's laws. In particular, oscillatory motions cannot exist. In this paper we show that oscillatory orbits do exist for any value of the mass ratio $\mu \in(0,1 / 2]$.

The study of oscillatory motions was started by Chazy (see AKN88). In 1922, he gave a complete classification of all possible states that a three body problem can approach as time tends to infinity (see Section 2.4 of AKN88). For the restricted three body problem (either planar or spatial, circular or elliptic) the possible final states are reduced to four:

- $H^{ \pm}$(hyperbolic): $\|q(t)\| \rightarrow \infty$ and $\|\dot{q}(t)\| \rightarrow c>0$ as $t \rightarrow \pm \infty$.

- $P^{ \pm}$(parabolic): $\|q(t)\| \rightarrow \infty$ and $\|\dot{q}(t)\| \rightarrow 0$ as $t \rightarrow \pm \infty$.

- $B^{ \pm}$(bounded): $\lim \sup _{t \rightarrow \pm \infty}\|q\|<+\infty$.

- $O S^{ \pm}$(oscillatory): $\lim \sup _{t \rightarrow \pm \infty}\|q\|=+\infty$ and $\liminf \operatorname{int}_{t \rightarrow \pm \infty}\|q\|<+\infty$.

Examples of all these types of motion, except the oscillatory ones, were already known by Chazy. The first to prove the existence of oscillatory motions was Sitnikov in Sit60. In his paper he considered the restricted spatial three body problem with mass ratio $\mu=1 / 2$ and the three bodies disposed in a certain symmetric configuration, called now the Sitnikov example. Later, Moser Mos73 gave a new proof. His approach to prove the existence of such motions was to consider the invariant manifolds of infinity and to prove that they intersect transversally. Then, he established the existence of symbolic dynamics close to these invariant manifolds which lead to the existence of oscillatory motions.

In the planar setting, the first result was by Simó and Llibre LS80a. Following the same approach as in Mos73, they proved the existence of oscillatory motions for the RPC3BP for small enough values 
of $\mu$. One of the main ingredients of their proof, as in [Mos73, was the study of the transversality of the intersection of the invariant manifolds of infinity. For $\mu=0$, the system is integrable and the stable and unstable invariant manifolds of infinity coincide. Then, they applied the classical Poincaré Melnikov Theory Poi90, Mel63 to ensure that, for $\mu$ small enough, the invariant manifolds do not coincide anymore and intersect transversally. Nevertheless, to be able to compute the Melnikov function, they considered the Jacobi constant $\mathcal{J}$ (see (2) ) large enough. For these values of the Jacobi constant, the Melnikov function is exponentially small with respect to $\mathcal{J}$ and therefore they were only able to prove the transversality of the invariant manifolds provided $\mu$ was exponentially small with respect to the Jacobi constant. This allowed them to prove the existence of oscillatory motions for small enough $\mu$. Note that the orbits that they obtained have large Jacobi constant, which implies that they are far from the primaries and, therefore, they are far from collisions. Their result was extended by Xia Xia92 using the real-analyticity of the invariant manifolds of infinity. He claimed that these invariant manifolds intersect transversally for any mass ratio $\mu \in(0,1 / 2]$ except for a finite number of values, and thus obtaining the existence of oscillatory motions for any mass ratio except for these values (see also Mos73 for a similar argument for the Sitnikov problem).

Following the same approach, in MP94 some formal computations were performed to show that, for the restricted planar elliptic three body problem, the invariant manifolds of infinity intersect transversally for arbitrarily small mass ratio. A rigorous computation of the Melnikov function is done in [DKdlRS12, assuming the eccentricity small enough. The existence of oscillatory motions has also been proven for the (non necessarily restricted) collinear three body problem LS80b. All the mentioned works follow the approach initially developed in Mos73, that is, they relate the oscillatory motions to transversal homoclinic points to infinity and symbolic dynamics. A completely different approach using AubryMather theory and semi-infinite regions of instability has been recently developed in GK11, GK10b, GK10a. This new approach has allowed the authors to prove the existence of orbits which initially are in the range of our Solar System and become oscillatory as time tends to infinity for the RPC3BP with a realistic mass ratio for the Sun-Jupiter pair.

The mentioned works deal with the problem of the existence of oscillatory motions in different models of Celestial Mechanics. Once the existence is known, the natural question is to measure how abundant they are. The only result in this direction is the recent paper [GK12, in which the authors study the Hausdorff dimension of the set of oscillatory motions for the Sitnikov example and the RPC3BP. Using Mos73 and LS80a, they prove that for both problems and a Baire generic subset of an open set of parameters (the eccentricity of the primaries in the Sitnikov example and the mass ratio and the Jacobi constant in the RPC3BP) the Hausdorff dimension is maximal. As pointed out to us by V. Kaloshin and A. Gorodetski, the present paper and the techniques developed by them in GK12 lead to the prove of the existence of a set of maximal Hausdorff dimension of oscillatory motions for any value of the mass ratio and a Baire generic subset of an open set of Jacobi constants.

The purpose of this paper is to improve the results of LS80a and Xia92. We prove, using $\mathcal{J}^{-1}$ as a perturbative parameter, that the transversality of the invariant manifolds of infinity holds for any value of $\mu \in(0,1 / 2]$ and the Jacobi constant $\mathcal{J}$ big enough. Moreover, we find a curve in the parameter plane $(\mu, \mathcal{J})$ where the stable and unstable invariant manifolds of infinity undergo a cubic homoclinic tangency. Note that in this setting, classical perturbative techniques such as Poincaré-Melnikov Theory do not apply because the difference between the stable and unstable invariant manifolds is exponentially small with respect to $\mathcal{J}$. That is, we have to face what is usually called the exponentially small splitting of separatrices phenomenon. This phenomenon was discovered by Poincaré [Poi90] while studying the nonintegrability of the $n$-body problem and he called it the Fundamental problem of mechanics. It has drawn considerable attention in the past decades but, due to its difficulty, it has essentially only been considered in toy models [HMS88, DS92, Tre97, Gel00, GOS10 or in general systems under hypothesis that typically do not apply when one wants to study problems from Celestial Mechanics [DS97, Gel97, BF04, BFGS11, Gua12. For instance, most of the results deal with Hamiltonian functions which are essentially either polynomials or trigonometric polynomials with respect to the state variables. The present paper is the first one which proves exponentially small splitting of separatrices in Celestial Mechanics and also the first one which deals with an irrational Hamiltonian without assuming artificial smallness conditions on the parameters of the problem. 
The main result of the present paper is the following.

Theorem 1.1 (Main Theorem: version 1). Fix any $\mu \in(0,1 / 2]$. Then, there exists an orbit $(q(t), p(t))$ of (11) which is oscillatory. Namely, it satisfies

$$
\limsup _{t \rightarrow \pm \infty}\|q\|=+\infty \quad \text { and } \quad \liminf _{t \rightarrow \pm \infty}\|q\|<+\infty
$$

As we have explained the RPC3BP has as a first integral the Jacobi constant (2) and we take it big enough. Thus, in fact we have a more precise knowledge of the oscillatory motions we obtain.

Theorem 1.2 (Main Theorem: version 2). Fix any $\mu \in(0,1 / 2]$. Then, there exists $J_{0}>0$ big enough, such that for any $J>J_{0}$ there exists an orbit $\left(q_{J}(t), p_{J}(t)\right)$ of (1) in the hypersurface $\mathcal{J}(q, p, t ; \mu)=J$ which is oscillatory. Namely, it satisfies

$$
\limsup _{t \rightarrow \pm \infty}\left\|q_{J}\right\|=+\infty \text { and } \liminf _{t \rightarrow \pm \infty}\left\|q_{J}\right\|<+\infty .
$$

Moreover, if we take $\mu^{*} \in(0,1 / 2)$, there exists $J^{*}>0$ big enough such that, for any $J>J^{*}$ and $\mu \in\left(0, \mu^{*}\right]$, the previous claims hold.

The main difficulty to prove these theorems is to show that the invariant manifolds of infinity intersect transversally. To state the corresponding result, we first need to set up some notation and formally define what the invariant manifolds of infinity are. First, writing the system in rotating coordinates it turns out to be a two degrees of freedom Hamiltonian system. At each energy level, infinity corresponds to a periodic orbit with two dimensional stable and unstable manifolds. Their intersection is studied in Section 2 First, Theorem 2.1 gives an asymptotic formula for their difference. Then, in Theorem 2.2 we see that, for any $\mu \in(0,1 / 2]$ and $\mathcal{J}$ big enough, they intersect transversally. Theorem 2.2 also gives a formula for the area of the lobes between two consecutive homoclinic points in an associated Poincaré map. Then, Theorems 1.1 and 1.2 follow from Theorem 2.2 using the reasoning in LS80a. Furthermore, we are able to detect a bifurcation curve in the parameter space $(\mu, J)$ where the transversality of the invariant manifolds is lost since a cubic homoclinic tangency appears. This fact is stated in Theorem 2.3 ,

The rest of the paper, that is Sections 36, is devoted to prove Theorems 2.1, 2.2 and 2.3. In Section 3 following LMS03, Sau01, we look for parameterizations of the invariant manifolds as graphs of generating functions in a suitable domain using the Hamilton-Jacobi equation and we state Theorem 3.2 which gives the difference of these generating functions. Then, Theorems 2.1 and 2.2 follow easily from Theorem 3.2. The prove of Theorem 2.3 is deferred to the end of Section 6

Theorem 3.2 is proved in the subsequent sections. First, in Section 4 , we state certain analytic properties of the unperturbed separatrix which are crucial to obtain solutions of the Hamilton-Jacobi equation. Then, in Section 5, we prove the existence of "solutions" of the Hamilton-Jacobi equation, which are periodic in one of their variables, in certain complex domains. The quotation marks refer to the fact that we do not obtain actual solutions of the Hamilton-Jacobi equation. Indeed, to proof exponentially small splitting of separatrices, usually one has to obtain parameterizations of the invariant manifolds in complex domains which reach a neighborhood of the singularities of the unperturbed separatrix (see for instance BFGS11). This is not possible in the present problem since the parameterizations blow up before reaching these neighborhoods of the singularities. Instead, we consider the Fourier series of the generating functions (see Section 5 for more details) and we show that, even if the parameterizations blow up, their Fourier coefficients are well defined in the corresponding complex domains. This turns out to be sufficient to study the exponentially small splitting of separatrices. Finally, in Section 6, we complete the proof of Theorem 3.2. Analyzing the difference between two solutions (as formal Fourier series) of the Hamilton-Jacobi equation, which correspond to the stable and unstable manifolds, in the complex domains, we deduce exponentially small estimates for the difference between the generating functions for real values of the variables. At the end of this section we also prove Theorem 2.3 . 


\section{The invariant manifolds of infinity}

To study the invariant manifolds of infinity, it is more convenient to express the Hamiltonian (10) in polar coordinates. It is given by

$$
\begin{aligned}
H(r, \alpha, y, G, t ; \mu) & =\frac{y^{2}}{2}+\frac{G^{2}}{2 r^{2}}-\widetilde{U}(r, \alpha-t ; \mu) \\
& =\frac{y^{2}}{2}+\frac{G^{2}}{2 r^{2}}-\frac{1}{r}-U(r, \alpha-t ; \mu),
\end{aligned}
$$

where $(r, \alpha)$ are the polar coordinates of the configuration space and $(y, G)$ are the symplectic conjugate variables. That is, $y$ is the radial velocity (or momentum) and $G$ is the angular momentum. The function $\widetilde{U}$ is the Newtonian potential, which is given by

$$
\widetilde{U}(r, \phi ; \mu)=\frac{1-\mu}{\left(r^{2}-2 \mu r \cos \phi+\mu^{2}\right)^{1 / 2}}+\frac{\mu}{\left(r^{2}+2(1-\mu) r \cos \phi+(1-\mu)^{2}\right)^{1 / 2}}
$$

and therefore $U$ satisfies $U=\mathcal{O}(\mu)$. Nevertheless, recall that we are considering any $\mu \in(0,1 / 2]$ and therefore $\mu$ is non necessarily small.

The associated equations are

$$
\begin{aligned}
\dot{r} & =y \\
\dot{y} & =\frac{G^{2}}{r^{3}}-\frac{1}{r^{2}}+\partial_{r} U(r, \alpha-t ; \mu) \\
\dot{\alpha} & =\frac{G}{r^{2}} \\
\dot{G} & =\partial_{\alpha} U(r, \alpha-t ; \mu) .
\end{aligned}
$$

Call $\Phi_{t, t_{0}}=\left(\Phi_{t, t_{0}}^{r}, \Phi_{t, t_{0}}^{y}, \Phi_{t, t_{0}}^{\alpha}, \Phi_{t, t_{0}}^{G}\right)$ to the flow associated to this equation. Then, the stable and unstable manifolds of infinity are defined as

$$
\begin{aligned}
& \mathcal{W}_{\infty}^{s}=\left\{(r, y, \alpha, G) \in \mathbb{R}^{2} \times \mathbb{T} \times \mathbb{R}: \lim _{t \rightarrow+\infty} \Phi_{t, t_{0}}^{r}(r, y, \alpha, G ; \mu)=\infty, \lim _{t \rightarrow+\infty} \Phi_{t, t_{0}}^{y}(r, y, \alpha, G ; \mu)=0\right\} \\
& \mathcal{W}_{\infty}^{u}=\left\{(r, y, \alpha, G) \in \mathbb{R}^{2} \times \mathbb{T} \times \mathbb{R}: \lim _{t \rightarrow-\infty} \Phi_{t, t_{0}}^{r}(r, y, \alpha, G ; \mu)=\infty, \lim _{t \rightarrow-\infty} \Phi_{t, t_{0}}^{y}(r, y, \alpha, G ; \mu)=0\right\},
\end{aligned}
$$

where $\mathbb{T}=\mathbb{R} /(2 \pi \mathbb{Z})$. It is known that these invariant manifolds are analytic (see McG73]).

It is well known that the RPC3BP possesses a symmetry which, in polar coordinates, means that the system (4) is reversible with respect to the involution

$$
\mathcal{R}(r, y, \alpha, G)=(r,-y,-\alpha, G)
$$

We will use this fact to obtain symmetric properties of the parameterizations of the invariant manifolds.

When $\mu=0$, the RPC3BP is reduced to a central force equation. This system is autonomous and therefore has conservation of energy $H$. Moreover, the angular momentum $G$ is also conserved and then the system is integrable. This implies that the invariant manifolds of infinity coincide along a homoclinic manifold, $\mathcal{W}_{\infty}^{s}=\mathcal{W}_{\infty}^{u}$. This manifold is formed by a family of homoclinic orbits to infinity which perform Keplerian parabolic orbits. Recall that by "infinity" we mean $(r, y)=(+\infty, 0)$ and then, "infinity" is foliated by periodic orbits which can be parameterized by the angular momentum $G$ and are of the form

$$
\Lambda_{G_{0}}=\left\{(r, \alpha, y, G): r=\infty, y=0, \alpha \in \mathbb{T}, G=G_{0}\right\} .
$$

All the associated homoclinic orbits belong to the energy level $H=0$ and can be parameterized by the angular momentum and their initial condition in the angular variable. We denote these orbits as

$$
z_{\mathrm{h}}\left(u ; G_{0}, \alpha_{0}\right)=\left(r_{\mathrm{h}}\left(u ; G_{0}\right), \alpha_{0}+\alpha_{\mathrm{h}}\left(u ; G_{0}\right), y_{\mathrm{h}}\left(u ; G_{0}\right), G_{0}\right),
$$


and we fix the origin of time such that $y_{\mathrm{h}}\left(0 ; G_{0}\right)=0$ and $\alpha_{\mathrm{h}}\left(0 ; G_{0}\right)=0$ (see Figure 1), which makes the homoclinic orbit with $\alpha_{0}=0$ symmetric, that is,

$$
\mathcal{R}\left(z_{\mathrm{h}}\left(u ; G_{0}, 0\right)\right)=z_{\mathrm{h}}\left(-u ; G_{0}, 0\right) .
$$

Commonly, in the study of this problem, one considers McGehee coordinates [McG73 $r=2 x^{-2}$ which send infinity to zero. In these new coordinates the system is still Hamiltonian with a non canonical symplectic form and the origin becomes a parabolic periodic orbit with stable and unstable invariant manifolds. Since the symplectic form is non canonical in McGehee coordinates, their use is rather cumbersome. In the present work we prefer to stick to the original variables, in which the invariant manifolds $\mathcal{W}_{\infty}^{u, s}$ can be characterized as above.

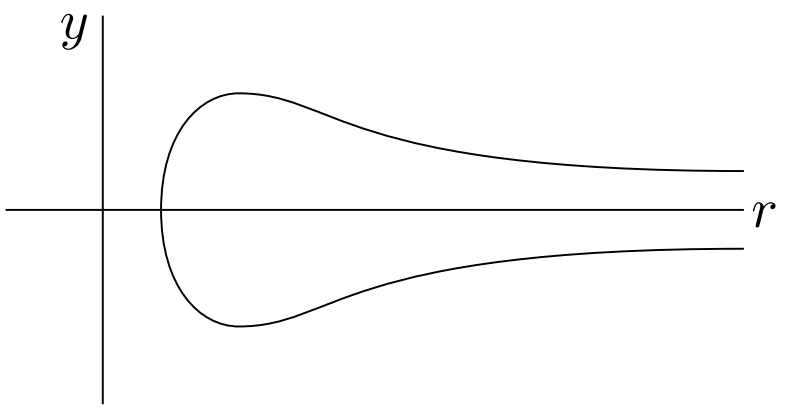

Figure 1: Projection on the $(r, y)$ plane of the separatrix (7) of system (4) with $\mu=0$. It also correspond to the separatrix of the Poincaré map $\mathcal{P}_{G_{0}, \phi_{0}}$ in (18).

Before studying these invariant manifolds, we perform some rescalings that make the perturbative character more apparent for big enough angular momentum $G_{0}$. In these new variables, the homoclinic (7) will be independent of $G_{0}$, which will facilitate the exposition of the results.

\subsection{The RPC3BP as a nearly integrable Hamiltonian System with two time scales}

It is well known that the existence of exponentially small phenomena usually arises when the system possesses two different time scales. More concretely, the exponentially small splitting of separatrices often appears when the system has combined elliptic and hyperbolic (or parabolic) behavior in such a way that the elliptic one is much faster than the hyperbolic (or parabolic) one. Even if this is not obvious looking at Hamiltonian (3), this is also the case in this problem. To make this fact more apparent we perform a change of variables which is simply a rescaling. It turns out that the main theorems of this section, Theorems 2.1, 2.2 and 2.3, are significantly simpler in the rescaled variables.

Before showing the rescalings, let us give some heuristic ideas why this system has two time scales. Taking arbitrarily high Jacobi constant implies that the massless body is extremely far from the primaries, that is, in a neighborhood of infinity in the configuration space. Moreover, recall that the oscillatory motions lie close to the invariant manifolds of infinity which are formed by orbits whose speed tends to zero as its position tends to infinity. These two facts together imply that the massless body has an extremely slow motion compared with the primaries. Therefore, after a suitable rescaling one has a massless body which has a speed of order one, which is perturbed by the motion of the primaries that now are rapidly rotating.

Let us recall that, even for system (4) neither $H$ nor $G$ are preserved as happened for the case $\mu=0$, there is still a conserved quantity: the Jacobi constant. In polar coordinates, it is given by

$$
\mathcal{J}(r, \alpha, y, G, t ; \mu)=H(r, \alpha, y, G, t ; \mu)-G
$$


We first fix it to be $\mathcal{J}=G_{0}$. Then, in this invariant hypersurface we perform the following changes of variables

$$
r=G_{0}^{2} \widetilde{r}, \quad y=G_{0}^{-1} \widetilde{y}, \quad \alpha=\widetilde{\alpha} \quad \text { and } \quad G=G_{0} \widetilde{G}
$$

and we rescale time as

$$
t=G_{0}^{3} s
$$

obtaining the system

$$
\begin{aligned}
\frac{d}{d s} \widetilde{r} & =\widetilde{y} \\
\frac{d}{d s} \widetilde{y} & =\frac{\widetilde{G}^{2}}{\widetilde{r}^{3}}-\frac{1}{\widetilde{r}^{2}}+G_{0}^{4} \partial_{r} U\left(G_{0}^{2} \widetilde{r}, \widetilde{\alpha}-G_{0}^{3} s ; \mu\right) \\
\frac{d}{d s} \widetilde{\alpha} & =\frac{\widetilde{G}}{\widetilde{r}^{2}} \\
\frac{d}{d s} \widetilde{G} & =G_{0}^{2} \partial_{\alpha} U\left(G_{0}^{2} \widetilde{r}, \widetilde{\alpha}-G_{0}^{3} s ; \mu\right) .
\end{aligned}
$$

Now the two time scales become clear. Indeed, in these variables we have that $d \widetilde{y} / d s \sim d \widetilde{r} / d s \sim 1$, which are the variables that will define the separatrix, whereas the perturbation dependence on time is fast. Calling

$$
V\left(\widetilde{r}, \phi ; \mu, G_{0}\right)=G_{0}^{2} U\left(G_{0}^{2} \widetilde{r}, \phi ; \mu\right),
$$

we have that

$$
V\left(\widetilde{r}, \phi ; \mu, G_{0}\right)=\frac{1-\mu}{\left(\widetilde{r}^{2}-2\left(\frac{\mu}{G_{0}^{2}}\right) \widetilde{r} \cos \phi+\left(\frac{\mu}{G_{0}^{2}}\right)^{2}\right)^{1 / 2}}+\frac{\mu}{\left(\widetilde{r}^{2}+2\left(\frac{1-\mu}{G_{0}^{2}}\right) \widetilde{r} \cos \phi+\left(\frac{1-\mu}{G_{0}^{2}}\right)^{2}\right)^{1 / 2}}-\frac{1}{\widetilde{r}},
$$

and we obtain the system we are going to study

$$
\begin{aligned}
\frac{d}{d s} \widetilde{r} & =\widetilde{y} \\
\frac{d}{d s} \widetilde{y} & =\frac{\widetilde{G}^{2}}{\widetilde{r}^{3}}-\frac{1}{\widetilde{r}^{2}}+\partial_{\widetilde{r}} V\left(\widetilde{r}, \widetilde{\alpha}-G_{0}^{3} s ; \mu, G_{0}\right) \\
\frac{d}{d s} \widetilde{\alpha} & =\frac{\widetilde{G}}{\widetilde{r}^{2}} \\
\frac{d}{d s} \widetilde{G} & =\partial_{\alpha} V\left(\widetilde{r}, \widetilde{\alpha}-G_{0}^{3} s ; \mu, G_{0}\right) .
\end{aligned}
$$

Note that $V \sim \mu G_{0}^{-2}$ and thus, since we are taking $G_{0} \gg 1$, we are dealing with a fast oscillating small perturbation. When one studies the splitting of separatrices phenomena in the resonances of nearly integrable Hamiltonian systems typically the perturbation has the same size as the integrable unperturbed system (see [Tre97, BFGS11, Gua12]). This is not the case in the present problem. This fact will facilitate the study of the difference between the invariant manifolds since we will not need to use inner equations and complex matching techniques or continuous averaging as were used in those papers.

The rescaling (9) is conformally symplectic and therefore the new system (12) is Hamiltonian with respect to

$$
\widetilde{H}\left(\widetilde{r}, \widetilde{\alpha}, \widetilde{y}, \widetilde{G}, s ; \mu, G_{0}\right)=\frac{\widetilde{y}^{2}}{2}+\frac{\widetilde{G}^{2}}{2 \widetilde{r}^{2}}-\frac{1}{\widetilde{r}}-V\left(\widetilde{r}, \widetilde{\alpha}-G_{0}^{3} s ; \mu, G_{0}\right) .
$$

The Jacobi constant is now $\mathcal{J}=G_{0}^{-2} \widetilde{H}-G_{0} \widetilde{G}$ and the periodic orbit at infinity is given by $(\widetilde{r}, \widetilde{\alpha}, \widetilde{y}, \widetilde{G})=$ $(\infty, \widetilde{\alpha}, 0,1)$, which belongs to the surface of Jacobi constant

$$
\mathcal{J}\left(G_{0}^{2} \widetilde{r}, \widetilde{\alpha}, G_{0}^{-1} \widetilde{y}, \widetilde{G}_{0} G, G_{0}^{3} s ; \mu\right)=-G_{0}
$$


One of the main advantages of this new set of coordinates is that the parameterization of the separatrix of the unperturbed system (7) in the rescaled variables,

$$
(\widetilde{r}, \widetilde{\alpha}, \widetilde{y}, \widetilde{G})=\left(\widetilde{r}_{\mathrm{h}}(s), \widetilde{\alpha}_{\mathrm{h}}(s), \widetilde{y}_{\mathrm{h}}(s), \widetilde{G}_{\mathrm{h}}(s)\right),
$$

is independent of $G_{0}$ (and also of $\mu$ ). In particular, $\widetilde{G}_{\mathrm{h}}(s) \equiv 1$. Moreover, note that after the rescaling we still have

$$
\widetilde{y}_{\mathrm{h}}(0)=0 \quad \text { and } \quad \widetilde{\alpha}_{\mathrm{h}}(0)=0,
$$

and therefore

$$
\widetilde{r}_{\mathrm{h}}(s)=\widetilde{r}_{\mathrm{h}}(-s), \widetilde{y}_{\mathrm{h}}(s)=-\widetilde{y}_{\mathrm{h}}(-s) \text { and } \widetilde{\alpha}_{\mathrm{h}}(s)=-\widetilde{\alpha}_{\mathrm{h}}(-s) \text {. }
$$

\subsection{Main result: intersection of the invariant manifolds}

Let the invariant manifolds $\widetilde{\mathcal{W}}_{\infty}^{s}$ and $\widetilde{\mathcal{W}}_{\infty}^{u}$ be the rescaling of the invariant manifolds $\mathcal{W}_{\infty}^{s}$ and $\mathcal{W}_{\infty}^{u}$ in (5). Their coincidence along the homoclinic manifold (14) for $\mu=0$ (equivalently, $G_{0}=\infty$ ) is due to the integrability of system (12) for this value of $\mu$. When $\mu>0$, these manifolds do not longer coincide but they intersect. To study the transversality of this intersection is the main goal of this section. Before stating Theorems 2.1. 2.2 and 2.3. we first need to explain how we measure the transversality of the invariant manifolds.

Note that the dependence on $s$ in (13) is only through $\widetilde{\alpha}-G_{0}^{-3} s$. Thus, one can eliminate the time dependence in the Hamiltonian by defining a new angle $\phi=\widetilde{\alpha}-G_{0}^{-3} s$. Then, we obtain the new Hamiltonian

$$
\mathcal{H}\left(\widetilde{r}, \phi, \widetilde{y}, \widetilde{G} ; \mu, G_{0}\right)=\frac{\widetilde{y}^{2}}{2}-G_{0}^{3} \widetilde{G}+\frac{\widetilde{G}^{2}}{2 \widetilde{r}^{2}}-\frac{1}{\widetilde{r}}-V\left(\widetilde{r}, \phi ; \mu, G_{0}\right),
$$

which is an autonomous two degrees of freedom Hamiltonian and therefore has conserved energy $\mathcal{H}$. The energy conservation corresponds to the conservation of the Jacobi constant in the original coordinates. In fact, $\mathcal{H}\left(\widetilde{r}, \widetilde{\alpha}-G_{0}^{3} s, \widetilde{y}, \widetilde{G} ; \mu, G_{0}\right)=G_{0}^{2} \mathcal{J}\left(G_{0}^{2} \widetilde{r}, \widetilde{\alpha}, G_{0}^{-1} \widetilde{y}, G_{0} \widetilde{G}, G_{0}^{3} s ; \mu\right)$. This new set of coordinates is usually called (rescaled) rotating polar coordinates since they are set in a rotating frame with respect to the primaries. Namely, in these new coordinates the primaries remain fixed at the horizontal axis while the massless body revolves around them, that is, these coordinates are the polar version of the synodic ones.

In these new coordinates, the invariant manifolds $\widetilde{\mathcal{W}}_{\infty}^{s}$ and $\widetilde{\mathcal{W}}_{\infty}^{u}$ are three dimensional. If one fixes a level of energy $\mathcal{H}\left(\widetilde{r}, \phi, \widetilde{y}, \widetilde{G} ; \mu, G_{0}\right)=-G_{0}^{3}$, the corresponding invariant manifolds

$$
\begin{aligned}
& \mathcal{W}_{\infty, G_{0}}^{u}=\widetilde{\mathcal{W}}_{\infty}^{u} \cap\left\{\mathcal{H}\left(\widetilde{r}, \phi, \widetilde{y}, \widetilde{G} ; \mu, G_{0}\right)=-G_{0}^{3}\right\} \\
& \mathcal{W}_{\infty, G_{0}}^{s}=\widetilde{\mathcal{W}}_{\infty}^{s} \cap\left\{\mathcal{H}\left(\widetilde{r}, \phi, \widetilde{y}, \widetilde{G} ; \mu, G_{0}\right)=-G_{0}^{3}\right\}
\end{aligned}
$$

are two dimensional. Moreover, if one takes the energy high enough, in a neighborhood of these invariant manifolds one has that $\dot{\phi} \neq 0$. This implies that the flow associated to the Hamiltonian (17) restricted to a level of energy $\mathcal{H}\left(\widetilde{r}, \phi, \widetilde{y}, \widetilde{G} ; \mu, G_{0}\right)=-G_{0}^{3}$ induces a Poincaré map

$$
\begin{aligned}
\mathcal{P}_{G_{0}, \phi_{0}}:\left\{\phi=\phi_{0}\right\} & \longrightarrow\left\{\phi=\phi_{0}+2 \pi\right\} \\
(r, y) & \mapsto \mathcal{P}_{G_{0}, \phi_{0}}(r, y) .
\end{aligned}
$$

This Poincaré map is two dimensional and is area preserving since the flow is Hamiltonian (more precisely the Poincaré map preserves the symplectic form $\Omega=d r \wedge d y$ ). Now the invariant manifolds become invariant curves $\gamma^{u, s}$ (see Figure 22). We focus on the part of these invariant manifolds with $y>0$ and we consider a parameterization of $\gamma^{u, s}$ of the form

$$
\begin{aligned}
& \widetilde{r}=\widetilde{r}_{\mathrm{h}}(v) \\
& \widetilde{y}=Y_{\phi_{0}}^{u, s}\left(v ; \mu, G_{0}\right),
\end{aligned}
$$

where $\widetilde{r}_{\mathrm{h}}(v)$ is the $\widetilde{r}$ component of the separatrix parameterization (14). Then, following Sau01, to measure the distance between the invariant manifolds along a section $\widetilde{r}=$ const, it suffices to measure 
the difference between the functions $Y_{\phi_{0}}^{u, s}$. Note that the used parameterization is equivalent to write the curves as graphs $\widetilde{y}=y^{u, s}(r)$. Nevertheless, we use the auxiliary parameter $v$ since it simplifies the formulas in Theorem 2.1. We also give, in Theorem 2.2, a measure of the area of the lobes that are formed between the invariant curves since it is a symplectic invariant (see Figure 21). Proceeding analogously, one could easily give an asymptotic formula for the angle between the curves at homoclinic points. Indeed, in Theorem 2.3. we show that if one take values of the parameters in certain curve $\eta$ in the plane $\left(\mu, G_{0}\right)$, the invariant curves $\gamma^{u, s}$ in (19), besides a transversal intersection, have a (cubic) homoclinic tangency.

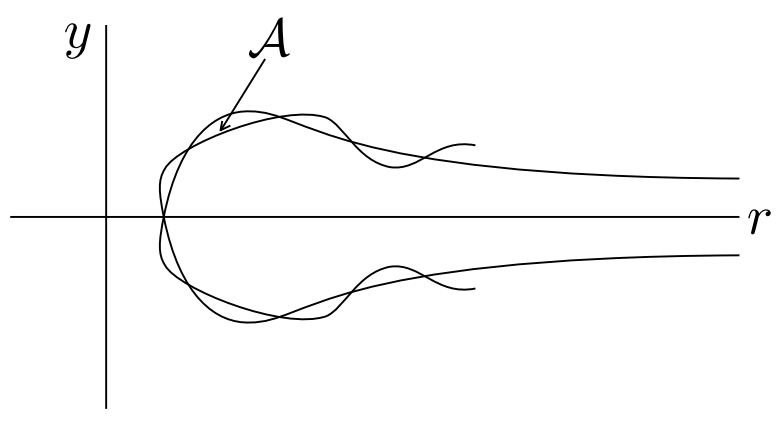

Figure 2: Stable and unstable invariant manifolds of infinity for the Poincaré map $\mathcal{P}_{G_{0}, \phi_{0}}$ in (18).

Theorem 2.1. Consider the invariant manifolds of infinity $\mathcal{W}_{\infty, G_{0}}^{s}$ and $\mathcal{W}_{\infty, G_{0}}^{u}$ of the system associated to Hamiltonian (17) in the level of energy $\mathcal{H}\left(\widetilde{r}, \phi, \widetilde{y}, \widetilde{G} ; \mu, G_{0}\right)=-G_{0}^{3}$ and the corresponding invariant curves $\gamma^{u, s}$ of the Poincaré map $\mathcal{P}_{G_{0}, \phi_{0}}$. Then, there exists $G_{0}^{*}>0$ such that for any $G_{0}>G_{0}^{*}$ and $\mu \in(0,1 / 2]$,

- the curves $\gamma^{u, s}$ have a parameterization of the form (19) and

- if we fix a section $\widetilde{r}=\widetilde{r}^{*}$, the distance d between these curves along this section is given by

$$
\begin{aligned}
d= & \widetilde{y}_{\mathrm{h}}\left(v^{*}\right)^{-1} \mu(1-\mu) \sqrt{\pi}\left[\frac{1-2 \mu}{2 \sqrt{2}} G_{0}^{3 / 2} e^{-\frac{G_{0}^{3}}{3}} \sin \left(\phi_{0}-\widetilde{\alpha}_{\mathrm{h}}\left(v^{*}\right)+G_{0}^{3} v^{*}\right)\right. \\
& +8 G_{0}^{7 / 2} e^{-\frac{2 G_{0}^{3}}{3}} \sin 2\left(\phi_{0}-\widetilde{\alpha}_{\mathrm{h}}\left(v^{*}\right)+G_{0}^{3} v^{*}\right) \\
& \left.+\mathcal{O}\left((1-2 \mu) G_{0} e^{-\frac{G_{0}^{3}}{3}}+G_{0}^{3} e^{-\frac{2 G_{0}^{3}}{3}}\right)\right],
\end{aligned}
$$

where $v^{*}$ is the only $v>0$ such that $\widetilde{r}\left(v^{*}\right)=\widetilde{r}^{*}$ and $\widetilde{y}_{\mathrm{h}}(v)$ and $\widetilde{\alpha}_{\mathrm{h}}(v)$ are the $\widetilde{y}$ and $\widetilde{\alpha}$ components of the unperturbed homoclinic (14).

This theorem is proven in Section 3 Observe that the distance is exponentially small with respect to $G_{0}$, which is taken as a large parameter. Moreover, note that the size of the first order in the formula of Theorem 2.1 is significantly different depending whether $\mu \neq 1 / 2$ or $\mu=1 / 2$. The reason is that Hamiltonian (17) is periodic with respect to $\phi$ with period $2 \pi$, in the first case, and $\pi$, in the second one. The physical explanation of the different periodicity goes as follows. For $\mu \neq 1 / 2$ (see left picture of Figure 3) the primaries rotate around the center of mass with the same period $2 \pi$ performing circles of different radius. On the contrary, when $\mu=1 / 2$ both bodies have the same mass and therefore, they move along the same circle where the two bodies are placed in diametrally opposed points (see right picture of Figure (3). Therefore, in the case $\mu=1 / 2$ the period of the system is the half of the period of the primaries around the center of mass. As usually happens in exponentially small splitting of separatrices phenomena, the smaller the period of the fast perturbation, the smaller the distance between the manifolds (see for instance [Nel84]). 


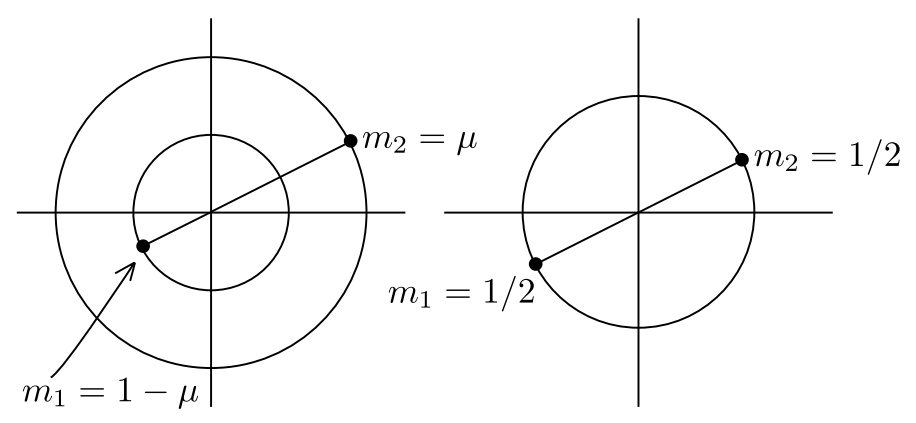

Figure 3: The motion of the primary bodies in the cases $\mu \neq 1 / 2$ and $\mu=1 / 2$ respectively.

Looking at the formula given in Theorem 2.1 the zeros of the distance are given, up to first order, by the zeros of the function

$$
f(x)=(1-2 \mu) \sin x+16 \sqrt{2} G_{0}^{2} e^{-\frac{G_{0}^{3}}{3}} \sin 2 x \text { where } x=\phi_{0}-\widetilde{\alpha}_{\mathrm{h}}(v)+G_{0}^{3} v .
$$

The number of zeros for $x \in[0,2 \pi)$ and their nondegeneracy depends strongly on the relation between the parameters $\mu$ and $G_{0}$. If we fix $\mu \neq 1 / 2$, and we take $G_{0}>0$ big enough, $f(x) \sim(1-2 \mu) \sin x$ and therefore we will have two nondegenerate zeros, which give rise to two transversal homoclinic points. On the contrary, for $\mu=1 / 2$ and $G_{0}$ big enough, $f(x) \sim 16 \sqrt{2} G_{0}^{2} e^{-G_{0}^{3} / 3} \sin 2 x$ and we will have four nondegenerate zeros. These facts are stated in Theorem 2.2. Clearly, between these two regimes the system undergoes a global bifurcation where one of the two transversal intersections becomes a cubic tangency where two new homoclinic points are born. This occurs in a curve $\eta$ in the parameter plane (see Figure 4) as is stated in Theorem 2.3.

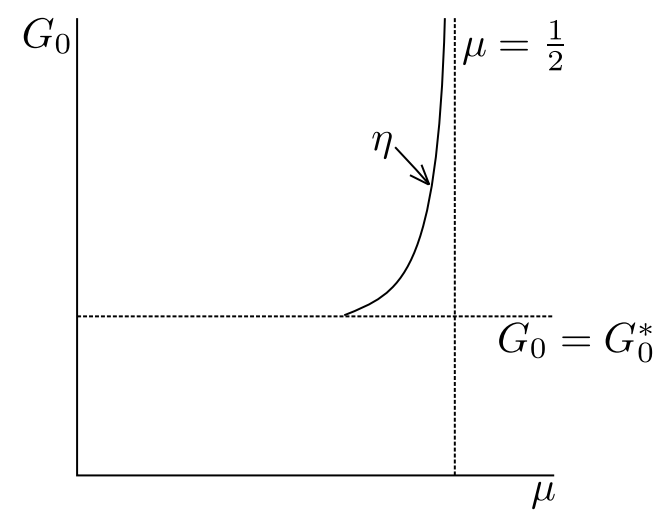

Figure 4: Bifurcation curve $\eta$ in the parameter space where the homoclinic tangency is undergone.

Theorem 2.2. Fix $\mu \in(0,1 / 2]$. Then there exists $G^{*}>0$ such that for any $G_{0}>G^{*}$,

- the invariant curves $\gamma^{u, s}$ of the Poincaré map $\mathcal{P}_{G_{0}, \phi_{0}}$ intersect transversally and

- the area of the lobes between the invariant curves $\gamma^{u, s}$ between two transversal consecutive homoclinic points is given by

$$
\mathcal{A}=\mu(1-\mu) \sqrt{\pi}\left[\frac{1-2 \mu}{\sqrt{2}} G_{0}^{-3 / 2} e^{-\frac{G_{0}^{3}}{3}}+8 G_{0}^{1 / 2} e^{-\frac{2 G_{0}^{3}}{3}}\right]\left(1+\mathcal{O}\left(G_{0}^{-1 / 2}\right)\right) .
$$


Theorem 2.3. Let $G_{0}^{*}>0$ be the constant introduced in Theorem 2.1. Then, there exists a curve $\eta$ in the parameter region

$$
\left(\mu, G_{0}\right) \in\left(0, \frac{1}{2}\right] \times\left(G_{0}^{*},+\infty\right)
$$

of the form

$$
\mu=\mu^{*}\left(G_{0}\right)=\frac{1}{2}-16 \sqrt{2} G_{0}^{2} e^{-\frac{G_{0}^{3}}{3}}\left(1+\mathcal{O}\left(G_{0}^{-1 / 2}\right)\right)
$$

such that, for $\left(\mu, G_{0}\right) \in \eta$,

- the invariant curves $\gamma^{u, s}$ of the Poincaré map $\mathcal{P}_{G_{0}, \phi_{0}}$ have a cubic homoclinic tangency and a transversal homoclinic point and

- the area of the lobes between the invariant curves $\gamma^{u, s}$ between the homoclinic tangency and a consecutive transversal homoclinic point is given by

$$
\mathcal{A}=10 \sqrt{\pi} G_{0}^{1 / 2} e^{-2 \frac{G_{0}^{3}}{3}}\left(1+\mathcal{O}\left(G_{0}^{-1 / 2}\right)\right) .
$$

Theorem 2.2 is proven in Section 3 . The proof of Theorem 2.3 is deferred to the end of Section 6 .

\subsection{From transversal homoclinic points to oscillatory motions}

The existence of oscillatory motions in the RPC3BP was obtained by Llibre and Simó in LS80a for large values of $G_{0}$ and exponentially small values of the mass ratio $\mu$, concretely for $\mu=\mathcal{O}\left(e^{-G_{0}{ }^{3} / 3}\right)$. Their arguments follow the ones developed by Moser in the so called Sitnikov problem in Mos73. The proof requires, as a first step, to control the local behavior near infinity. This is done using McGehee coordinates McG73], in which infinity becomes a parabolic critical point at the origin, and then the so-called Shilnikov coordinates to study the local map near zero.

The local behavior of the system at infinity turns out to be the same for all values of the parameter $\mu \in[0,1 / 2]$ and $G_{0}$ big enough. The reason is that when one considers system (44), makes the change of variables $\phi=\alpha-t$ to express it in rotating coordinates, performs the McGehee change of coordinates $r=2 x^{-2}$, uses the energy reduction and reparameterizes time $s=s(\theta)$ to have $d \phi / d \theta=1$, one obtains (see [LS80a]) the system

$$
\begin{aligned}
& \frac{d x}{d \theta}=\frac{x^{3} y}{4}+K \frac{x^{7} y}{32}+\mathcal{O}_{10}(x, y) \\
& \frac{d y}{d \theta}=\frac{x^{4}}{4}-K^{2} \frac{x^{6}}{32}+3 K \frac{x^{6} y^{2}}{16}-\lambda(\theta ; \mu) x^{8}+\mathcal{O}_{10}(x, y)
\end{aligned}
$$

where $K=\mathcal{J}-\mu(1-\mu)$ and $\lambda(\theta ; \mu)=\frac{3}{32} \mu(1-\mu)\left(1-3 \cos ^{2} \theta\right)$. Since the dependence on $\mu$ only appears in the higher order terms, the local behavior is the same regardless the value of the parameter $\mu \in[0,1 / 2]$. Therefore, the techniques developed by Moser in Mos73] that were applied in [LS80a for the case $\mu$ small enough, are also valid in our setting.

The second step of the proof is to show that the invariant stable and unstable manifolds of infinity intersect transversally. It was in this step that the smallness of the parameter $\mu$ was needed in [LS80a]. Theorem 2.2 guarantees this transversality for all values of $\mu$.

Hence combining this fact with the local results of [LS80a] we obtain Theorem 6.1 of [LS80a] for any value of $\mu \in(0,1 / 2]$, which shows that there exists a Cantor set in the phase space where certain return map associated to system (4) is conjugated with a shift of infinitely many symbols. From this result, Theorems 1.1 and 1.2 follow directly.

Another way of proving Theorem 1.1 and 1.2 would be to consider the Poincaré map (18) in McGehee coordinates, which has a parabolic fixed point at the origin with transversal homoclinic points. Then, as Moser mentions in Mos73 for the Sitnikov problem, one could adapt the proof of the classical BirkhoffSmale theorem to parabolic fixed points with transversal homoclinic intersections to obtain a Cantor set of the phase space with dynamics topologically conjugated to a shift of two symbols. Using this idea 
and proving a $\mathcal{C}^{2}$-version of the Lambda Lemma, Gorodetski and Kaloshin in the recent preprint GK12, prove that the Hausdorff dimension of the set of initial conditions which lead to oscillatory motions is maximal. Their proof also needs the same requirements about the parameters $\mu$ and $G_{0}$ of LS80a because their reasoning uses the transversality of the invariant manifolds proved in LS80a. Private conversations with the authors indicate that, using Theorem 2.2, their results can be extended for a larger set of the parameters.

\section{Parameterizations of the invariant manifolds as generating functions}

As we have explained in Section 2.1, to prove Theorems 2.1, 2.2 and 2.3 we consider the rescaled system in rotating coordinates given by Hamiltonian (17). The main two advantages of this choice are the following. First, the unperturbed separatrix is independent of the parameter $G_{0}$ and, second, working in rotating coordinates our system has two degrees of freedom (and thus conserved energy) instead of two and a half as happens in the original variables.

To study the difference between the invariant manifolds of infinity, we follow the approach proposed in LMS03, Sau01. That is, we take advantage of the fact that the invariant manifolds are Lagrangian and therefore they can be locally parameterized as graphs of generating functions which are solutions of the so-called Hamilton-Jacobi equation.

We consider the Hamilton-Jacobi equation associated to Hamiltonian (17) and we look for functions $S\left(\widetilde{r}, \phi ; \mu, G_{0}\right)$ such that

$$
(\widetilde{y}, \widetilde{G})=\left(\partial_{\widetilde{r}} S\left(\widetilde{r}, \phi ; \mu, G_{0}\right), \partial_{\phi} S\left(\widetilde{r}, \phi ; \mu, G_{0}\right)\right)
$$

parameterize the invariant manifolds as a graph. Then, the Hamilton-Jacobi equation reads

$$
\mathcal{H}\left(\widetilde{r}, \phi, \partial_{\widetilde{r}} S, \partial_{\phi} S ; \mu, G_{0}\right)=-G_{0}^{3} .
$$

Recall that we put $-G_{0}^{3}$ in the right hand side since it is the level of energy where we are looking for the invariant manifolds.

For the unperturbed Hamiltonian, that is, considering $V=0$ in (17), this equation simply reads

$$
\frac{1}{2}\left(\partial_{\widetilde{r}} S\right)^{2}-G_{0}^{3} \partial_{\phi} S+\frac{1}{2 \widetilde{r}^{2}}\left(\partial_{\phi} S\right)^{2}-\frac{1}{\widetilde{r}}=-G_{0}^{3} .
$$

It has a solution of the form

$$
S_{0}(\widetilde{r}, \phi)=\phi+f(\widetilde{r}),
$$

where $f$ is any solution of

$$
\frac{1}{2}\left(\partial_{\widetilde{r}} f\right)^{2}+\frac{1}{2 \widetilde{r}^{2}}-\frac{1}{\widetilde{r}}=0 .
$$

In LMS03, Sau01, the authors deal with unperturbed separatrices which can be written globally as graphs. This is not possible in the present problem, as can be clearly seen in Figure 1, since the separatrix has a turning point at $(\widetilde{r}, \widetilde{y})=(1 / 2,0)$. Thus, we will deal with this equation for $\widetilde{r} \neq 1 / 2$. This fact will carry some technical problems while proving the existence of the invariant manifolds in certain domains later in Section 5 ,

We look for solutions of (20) close to (21). To this end, we write $S=S_{0}+S_{1}$ and then the equation for $S_{1}$ becomes

$$
\partial_{\widetilde{r}} f \partial_{\widetilde{r}} S_{1}+\frac{1}{2}\left(\partial_{\widetilde{r}} S_{1}\right)^{2}-G_{0}^{3} \partial_{\phi} S_{1}+\frac{1}{\widetilde{r}^{2}} \partial_{\phi} S_{1}+\frac{1}{2 \widetilde{r}^{2}}\left(\partial_{\phi} S_{1}\right)^{2}-V\left(\widetilde{r}, \phi ; \mu, G_{0}\right)=0 .
$$

To look for solutions of this equation we proceed as in LMS03, Sau01 and we reparameterize the variables $(\widetilde{r}, \phi)$ through the unperturbed separatrix (14). Namely, we consider the change

$$
(\widetilde{r}, \phi)=\left(\widetilde{r}_{\mathrm{h}}(v), \xi+\widetilde{\alpha}_{\mathrm{h}}(v)\right) .
$$


We define the new generating function

$$
T\left(v, \xi ; \mu, G_{0}\right)=S\left(\widetilde{r}_{\mathrm{h}}(v), \xi+\widetilde{\alpha}_{\mathrm{h}}(v) ; \mu, G_{0}\right),
$$

which can be correspondingly written as $T=T_{0}+T_{1}$ where

$$
T_{0}(v, \xi)=S_{0}\left(\widetilde{r}_{\mathrm{h}}(v), \xi+\widetilde{\alpha}_{\mathrm{h}}(v)\right)
$$

and

$$
T_{1}\left(v, \xi ; \mu, G_{0}\right)=S_{1}\left(\widetilde{r}_{\mathrm{h}}(v), \xi+\widetilde{\alpha}_{\mathrm{h}}(v) ; \mu, G_{0}\right),
$$

whose associated Hamilton-Jacobi equation is

$$
\partial_{v} T_{1}-G_{0}^{3} \partial_{\xi} T_{1}+\frac{1}{2 \widetilde{y}_{\mathrm{h}}^{2}}\left(\partial_{v} T_{1}-\frac{1}{\widetilde{r}_{\mathrm{h}}^{2}} \partial_{\xi} T_{1}\right)^{2}+\frac{1}{2 \widetilde{r}_{\mathrm{h}}^{2}}\left(\partial_{\xi} T_{1}\right)^{2}-V\left(\widetilde{r}_{\mathrm{h}}, \xi+\widetilde{\alpha}_{\mathrm{h}} ; \mu, G_{0}\right)=0 .
$$

Note that the change of variables (22) implies that we are looking for parameterizations of the stable and unstable invariant manifolds of the form

$$
\begin{aligned}
\widetilde{r} & =\widetilde{r}_{\mathrm{h}}(v) \\
\widetilde{y} & =\widetilde{y}_{\mathrm{h}}(v)+\widetilde{y}_{\mathrm{h}}(v)^{-1}\left(\partial_{v} T_{1}^{u, s}\left(v, \xi ; \mu, G_{0}\right)-\widetilde{r}_{\mathrm{h}}(v)^{-2} \partial_{\xi} T_{1}^{u, s}\left(v, \xi ; \mu, G_{0}\right)\right) \\
\phi & =\xi+\widetilde{\alpha}_{\mathrm{h}}(v) \\
\widetilde{G} & =1+\partial_{\xi} T_{1}^{u, s}\left(v, \xi ; \mu, G_{0}\right),
\end{aligned}
$$

where $T_{1}^{u, s}$ are solutions of equation (24) with asymptotic boundary conditions for the unstable manifold

$$
\begin{aligned}
& \lim _{v \rightarrow-\infty} \widetilde{y}_{\mathrm{h}}^{-1}(v) \partial_{v} T_{1}^{u}\left(v, \xi ; \mu, G_{0}\right)=0 \\
& \lim _{v \rightarrow-\infty} \partial_{\xi} T_{1}^{u}\left(v, \xi ; \mu, G_{0}\right)=0,
\end{aligned}
$$

and analogous ones for the stable manifold taking $v \rightarrow+\infty$. By its definition in (11), $V$ has the symmetry property

$$
V(r,-\theta)=V(r, \theta) .
$$

Taking into account this fact and (16), one can easily see that if $T_{1}(v, \xi)$ is a solution of (24),$-T_{1}(-v,-\xi)$ is also a solution. Thus, since $T_{1}^{u}$ has to satisfy the just mentioned asymptotic boundary conditions and $T_{1}^{s}$ the opposite ones, the generating functions parameterizing the invariant manifolds must satisfy

$$
T_{1}^{s}(v, \xi)=-T_{1}^{u}(-v,-\xi) .
$$

This means that if one is able to prove the existence of, for instance, the unstable invariant manifold, the existence of the stable one is guaranteed by the symmetry.

Theorems 2.1 and 2.2 will be deduced from the study of the difference of the generating functions $T_{1}^{u}$ and $T_{1}^{s}$ and from the difference between their derivatives given in Theorem 3.2 below. The proof of Theorem 2.3 is postponed to the end of Section 6 . since we will need a slight modification of these generating functions to deduce the existence of tangencies.

It is worth remarking that, in view of (15) and the second equation of (25), the parameterizations are undefined at $v=0$ and therefore it seems impossible to have the parameterizations of both the stable and unstable manifold defined in a common domain. Nevertheless, in Section 5, we overcome this problem by using auxiliary parameterizations of the unstable manifold which allow us, later on, to recover parameterizations of both invariant manifolds of the form (25) in a common compact domain contained in $\{v>0\} \times \mathbb{T}$, which in the original variables corresponds to $y>0$ (see Figure 2).

Before stating Theorem 3.2, we define the function that will give the first asymptotic order between the generating functions $T_{1}^{u, s}$. This function is closely related to the so-called Poincaré function or Melnikov potential. We define

$$
L\left(v, \xi ; \mu, G_{0}\right)=\int_{-\infty}^{+\infty} V\left(\widetilde{r}_{\mathrm{h}}(v+s), \xi-G_{0}^{3} s+\widetilde{\alpha}_{\mathrm{h}}(v+s) ; \mu, G_{0}\right) d s,
$$


where $V$ is the rescaled perturbed Newtonian potential defined in (11). Note that the classical Poincaré function is the first order in $\mu$ of this function, that is

$$
\left.\int_{-\infty}^{+\infty} \partial_{\mu} V\left(\widetilde{r}_{\mathrm{h}}(v+s), \xi-G_{0}^{3} s+\widetilde{\alpha}_{\mathrm{h}}(v+s) ; \mu, G_{0}\right)\right|_{\mu=0} d s .
$$

This was the function considered as a first order of the difference between the invariant manifolds of infinity in LS80a, since they were considering perturbative methods in $\mu$. On the contrary, in the present paper $\mu$ can take any value $\mu \in(0,1 / 2]$ and therefore we deal with the modified Poincaré function (28). The following proposition, whose proof is deferred to Appendix A, gives estimates for this function.

Proposition 3.1. The function $L\left(v, \xi ; \mu, G_{0}\right)$ satisfies

$$
L\left(v, \xi ; \mu, G_{0}\right)=L^{[0]}\left(\mu, G_{0}\right)+2 \sum_{\ell=1}^{+\infty} L^{[\ell]}\left(\mu, G_{0}\right) \cos \ell\left(\xi+G_{0}^{3} v\right),
$$

where

$$
\begin{aligned}
& L^{[1]}\left(\mu, G_{0}\right)=-\mu(1-\mu) \sqrt{\pi} \frac{1-2 \mu}{4 \sqrt{2}} G_{0}^{-3 / 2} e^{-\frac{G_{0}^{3}}{3}}\left(1+\mathcal{O}\left(G_{0}^{-2}\right)\right) \\
& L^{[2]}\left(\mu, G_{0}\right)=-2 \mu(1-\mu) \sqrt{\pi} G_{0}^{1 / 2} e^{-\frac{2 G_{0}^{3}}{3}}\left(1+\mathcal{O}\left(G_{0}^{-1 / 2}\right)\right) \\
& L^{[\ell]}\left(\mu, G_{0}\right)=\left(G_{0}^{\ell-3 / 2} e^{-\frac{\ell G_{0}^{3}}{3}}\right), \text { for } \ell \geq 3 .
\end{aligned}
$$

Theorem 3.2. There exist $0<v_{-}<v_{+}, G_{0}^{*}>0$ and $K>0$ such that, for any $G_{0}>G_{0}^{*}$ and $\mu \in(0,1 / 2]$, the invariant manifolds of infinity have parameterizations of the form (25) for $(v, \xi) \in\left(v_{-}, v_{+}\right) \times \mathbb{T}$.

Moreover, the corresponding generating functions satisfy

$$
\left|T_{1}^{u}(v, \xi)-T_{1}^{s}(v, \xi)-L(v, \xi)-E\right| \leq K \mu^{2}(1-2 \mu) G_{0}^{-2} e^{\frac{G_{0}^{-3}}{3}}+K G_{0}^{-1 / 2} \mu^{2} e^{\frac{2 G_{0}^{-3}}{3}},
$$

for a constant $E \in \mathbb{R}$, which might depend on $\mu$ and $G_{0}$, and

$$
\left|\partial_{v}^{m} \partial_{\xi}^{n} T_{1}^{u}(v, \xi)-\partial_{v}^{m} \partial_{\xi}^{n} T_{1}^{s}(v, \xi)-\partial_{v}^{m} \partial_{\xi}^{n} L(v, \xi)\right| \leq K \mu^{2}(1-2 \mu) G_{0}^{-2+3 m} e^{\frac{G_{0}^{-3}}{3}}+K G_{0}^{-1 / 2+3 m} \mu^{2} e^{\frac{2 G_{0}^{-3}}{3}}
$$

for $0<m+n \leq 2,0 \leq m, n$, where we have omitted the dependence on $\mu$ and $G_{0}$ of $T_{1}^{u, s}, L$ and $E$ to simplify the notation.

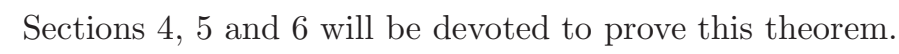

\subsection{Proof of Theorems 2.1 and 2.2}

We use the estimates obtained in Proposition 3.1 and Theorem 3.2 to prove Theorems 2.1 and 2.2. The first step is to deduce parameterizations (19) of the stable and unstable invariant curves of infinity $\gamma^{u, s}$ of the Poincaré map $\mathcal{P}_{G_{0}, \phi_{0}}$ in (18) from the generating functions $T_{1}^{u, s}$ obtained in Theorem 3.2 Recall that, on the one hand, the generating functions $T_{1}^{u, s}$ give us a parameterization of the invariant manifolds of infinity associated to Hamiltonian (17) of the form (25). On the other hand, the Poincaré section we are considering is $\phi=\phi_{0}$, which has natural coordinates $(r, y)$ (see (18)). This implies that to obtain the parameterization (19) of the invariant curves $\gamma^{u, s}$, one has to impose $\xi+\widetilde{\alpha}_{\mathrm{h}}(v)=\phi_{0}$ obtaining

$$
\begin{aligned}
Y_{\phi_{0}}^{u, s}\left(v ; \mu, G_{0}\right)= & \widetilde{y}_{\mathrm{h}}(v) \\
& +\left.\widetilde{y}_{\mathrm{h}}(v)^{-1}\left(\partial_{v} T_{1}^{u, s}\left(v, \xi ; \mu, G_{0}\right)-\widetilde{r}_{\mathrm{h}}(v)^{-2} \partial_{\xi} T_{1}^{u, s}\left(v, \xi ; \mu, G_{0}\right)\right)\right|_{\xi=\phi_{0}-\widetilde{\alpha}_{\mathrm{h}}(v)} .
\end{aligned}
$$


Using the estimates of Proposition 3.1 and Theorem 3.2 one has that

$$
\begin{aligned}
Y_{\phi_{0}}^{s}\left(v ; \mu, G_{0}\right)-Y_{\phi_{0}}^{u}\left(v ; \mu, G_{0}\right)= & \left.\widetilde{y}_{\mathrm{h}}(v)^{-1}\left(\partial_{v} T_{1}^{s}\left(v, \xi ; \mu, G_{0}\right)-\partial_{v} T_{1}^{u}\left(v, \xi ; \mu, G_{0}\right)\right)\right|_{\xi=\phi_{0}-\widetilde{\alpha}_{\mathrm{h}}(v)} \\
& +\mathcal{O}\left(\mu G_{0}^{-3 / 2} e^{-\frac{G_{0}^{-3}}{3}}\right) \\
= & \widetilde{y}_{\mathrm{h}}(v)^{-1} \partial_{v} L\left(v, \phi_{0}-\widetilde{\alpha}_{\mathrm{h}}(v) ; \mu, G_{0}\right) \\
& +\mathcal{O}\left(\mu^{2}(1-2 \mu) G_{0} e^{-\frac{G_{0}^{-3}}{3}}+G_{0}^{5 / 2} \mu^{2} e^{\frac{2 G_{0}^{-3}}{3}}\right)
\end{aligned}
$$

Now taking into account Proposition 3.1 we obtain the formula of the distance in Theorem 2.1

To prove Theorem 2.2, we first fix $\mu \in(0,1 / 2)$. Then, taking $G_{0}>G^{*}, G^{*}=G^{*}(\mu)$ big enough,

$$
\begin{aligned}
Y_{\phi_{0}}^{s}\left(v ; \mu, G_{0}\right)-Y_{\phi_{0}}^{u}\left(v ; \mu, G_{0}\right)= & -\widetilde{y}_{\mathrm{h}}(v)^{-1} \mu(1-\mu) \sqrt{\pi} \frac{2 \mu-1}{2 \sqrt{2}} G_{0}^{3 / 2} e^{-\frac{G_{0}^{3}}{3}} \sin \left(\phi_{0}-\widetilde{\alpha}_{\mathrm{h}}(v)+G_{0}^{3} v\right) \\
& +\mathcal{O}\left(\mu G_{0} e^{-\frac{G_{0}^{3}}{3}}\right) .
\end{aligned}
$$

This gives the formula of the distance for fixed $\mu \in(0,1 / 2)$. Proceeding analogously, one obtains the following formula for $\mu=1 / 2$ and $G_{0}$ big enough,

$$
\begin{aligned}
Y_{\phi_{0}}^{s}\left(v ; 1 / 2, G_{0}\right)-Y_{\phi_{0}}^{u}\left(v ; 1 / 2, G_{0}\right)= & \widetilde{y}_{\mathrm{h}}(v)^{-1} \sqrt{\pi} G_{0}^{7 / 2} e^{-\frac{2 G_{0}^{3}}{3}} \sin 2\left(\phi_{0}-\widetilde{\alpha}_{\mathrm{h}}(v)+G_{0}^{3} v\right) \\
& +\mathcal{O}\left(G_{0}^{3} e^{-\frac{2 G_{0}^{3}}{3}}\right) .
\end{aligned}
$$

We prove the transversality of the invariant manifolds and compute the area of the lobes for the case $\mu \neq 1 / 2$. The homoclinic points of infinity are just given by the equation

$$
Y_{\phi_{0}}^{s}\left(v ; \mu, G_{0}\right)-Y_{\phi_{0}}^{u}\left(v ; \mu, G_{0}\right)=0 .
$$

To locate the zeros of this equation we use the just obtained first order of the difference of the function $Y_{\phi_{0}}^{u, s}(v)$. The zeros of this first order correspond to the zeros of $\sin \left(\phi_{0}-\widetilde{\alpha}_{\mathrm{h}}(v)+G_{0}^{3} v\right)$ and thus are $v_{k}^{0} \in \mathbb{R}$ such that

$$
\phi_{0}-\widetilde{\alpha}_{\mathrm{h}}\left(v_{k}^{0}\right)+G_{0}^{3} v_{k}^{0}=k \pi, \quad k \in \mathbb{Z},
$$

which are $G_{0}^{-3}$-close to each other. These zeros are non degenerate since for $G_{0}$ big enough $\widetilde{\alpha}_{\mathrm{h}}^{\prime}(v)+G_{0}^{3} \neq 0$.

Using the estimates of Proposition 3.1 and Theorem 3.2 and taking $G_{0}$ large enough, one can apply the Implicit Function Theorem to show that the function $Y_{\phi_{0}}^{s}(v)-Y_{\phi_{0}}^{u}(v)$ has nondegenerate zeros $v_{k}^{1} \in \mathbb{R}$ which satisfy

$$
v_{k}^{1}=v_{k}^{0}+\mathcal{O}\left(G_{0}^{-7 / 2}\right),
$$

and therefore the curves $\gamma^{u}$ and $\gamma^{s}$ intersect transversally at the corresponding homoclinic points.

To compute the asymptotic formula for the area of the lobes, we proceed as in BFGS11 and we consider the change of coordinates

$$
\left\{\begin{array}{l}
r=\widetilde{r}_{\mathrm{h}}(v) \\
y=\widetilde{y}_{\mathrm{h}}(v)^{-1} w .
\end{array}\right.
$$

Since $\partial_{v} \widetilde{r}_{\mathrm{h}}(v)=\widetilde{y}_{\mathrm{h}}(v)$, we have that $d r \wedge d y=d v \wedge d w$. We compute the area of the lobes in this new system of coordinates. Now the invariant curves are parameterized as a graph as

$$
w=\widetilde{y}_{\mathrm{h}}(v) Y_{\phi_{0}}^{u, s}\left(v ; \mu, G_{0}\right) .
$$


Consider two consecutive zeros which we denote by $v_{k}^{1}$ and $v_{k+1}^{1}$. Then, using the definition of the function $Y_{\phi_{0}}^{u, s}$ in (29) and that $\widetilde{\alpha}_{\mathrm{h}}^{\prime}(v)=1 / \widetilde{r}_{\mathrm{h}}^{2}(v)$ (see equation (12) with $\mu=0$ ), the area of the lobes is given by

$$
\begin{aligned}
\mathcal{A}= & \left|\int_{v_{k}^{1}}^{v_{k+1}^{1}} \widetilde{y}_{\mathrm{h}}(v)\left(Y_{\phi_{0}}^{s}\left(v ; \mu, G_{0}\right)-Y_{\phi_{0}}^{u}\left(v ; \mu, G_{0}\right)\right) d v\right| \\
=\mid & \mid \int_{v_{k}^{1}}^{v_{k+1}^{1}}\left(\left(\partial_{v} T_{1}^{s}\left(v, \xi ; \mu, G_{0}\right)-\widetilde{r}_{\mathrm{h}}(v)^{-2} \partial_{\xi} T_{1}^{u}\left(v, \xi ; \mu, G_{0}\right)\right)\right. \\
& \left.\quad-\left(\partial_{v} T_{1}^{s}\left(v, \xi ; \mu, G_{0}\right)-\widetilde{r}_{\mathrm{h}}(v)^{-2} \partial_{\xi} T_{1}^{u}\left(v, \xi ; \mu, G_{0}\right)\right)\right)\left.\right|_{\xi=\phi_{0}-\widetilde{\alpha}_{\mathrm{h}}(v)} d v \mid \\
= & \mid\left(T_{1}^{s}\left(v_{k+1}^{1}, \phi_{0}-\widetilde{\alpha}_{\mathrm{h}}\left(v_{k+1}^{1}\right) ; \mu, G_{0}\right)-T_{1}^{u}\left(v_{k+1}^{1}, \phi_{0}-\widetilde{\alpha}_{\mathrm{h}}\left(v_{k+1}^{1}\right) ; \mu, G_{0}\right)\right) \\
& -\left(T_{1}^{s}\left(v_{k}^{1}, \phi_{0}-\widetilde{\alpha}_{\mathrm{h}}\left(v_{k}^{1}\right) ; \mu, G_{0}\right)-T_{1}^{u}\left(v_{k}^{1}, \phi_{0}-\widetilde{\alpha}_{\mathrm{h}}\left(v_{k}^{1}\right) ; \mu, G_{0}\right)\right) \mid .
\end{aligned}
$$

Then, applying Theorem 3.2 we have that

$$
\mathcal{A}=L\left(v_{k+1}^{1}, \phi_{0}-\widetilde{\alpha}_{\mathrm{h}}\left(v_{k+1}^{1}\right) ; \mu, G_{0}\right)-L\left(v_{k}^{1}, \phi_{0}-\widetilde{\alpha}_{\mathrm{h}}\left(v_{k}^{1}\right) ; \mu, G_{0}\right)+\mathcal{O}\left(\mu^{2} G_{0}^{-2} e^{\frac{G_{0}^{-3}}{3}}\right) .
$$

To obtain the formula for the area of the lobes in Theorem 2.2 it is enough to take into account the estimates in Proposition 3.1, that the zeros $v_{k}^{1}$ satisfy (31) and that the zeros of the first order $v_{k}^{0}$ satisfy (30).

The case $\mu=1 / 2$ can be done analogously but taking into account that now the zeros of the first order $v_{k}^{0}$ are solutions of

$$
\phi_{0}-\widetilde{\alpha}_{\mathrm{h}}\left(v_{k}^{0}\right)+G_{0}^{3} v_{k}^{0}=k \frac{\pi}{2}, \quad k \in \mathbb{Z} .
$$

\section{The integrable system and the homoclinic solution}

To study the existence of the solutions of the Hamilton-Jacobi equation (24), we need to recall some analytic properties of the functions $\widetilde{r}_{\mathrm{h}}, \widetilde{y}_{\mathrm{h}}$ and $\widetilde{\alpha}_{\mathrm{h}}$, which are solution of

$$
\begin{aligned}
& \frac{d}{d v} \widetilde{r}=\widetilde{y} \\
& \frac{d}{d v} \widetilde{y}=\frac{1}{\widetilde{r}^{3}}-\frac{1}{\widetilde{r}^{2}} \\
& \frac{d}{d v} \widetilde{\alpha}=\frac{1}{\widetilde{r}^{2}},
\end{aligned}
$$

close to its singularities and as $|v| \rightarrow+\infty$.

Next lemma (see [LS80a, MP94]) gives the homoclinic solution of infinity of these equations satisfying (15) after a reparameterization of time.

Lemma 4.1. Let $\tau(v)$ be the unique analytic function defined by

$$
v=\frac{1}{2}\left(\frac{1}{3} \tau^{3}+\tau\right)
$$

with the convention that $\tau$ is real for real values of $v$, that is,

$$
\tau(v)=\left(3 v+\sqrt{9 v^{2}+1}\right)^{1 / 3}-\left(3 v+\sqrt{9 v^{2}+1}\right)^{-1 / 3} .
$$

. Then, the rescaled homoclinic (14) satisfying (15) has the following properties: 
1. $\widetilde{r}(v)=\widehat{r}(\tau(v))$, where

$$
\widehat{r}(\tau)=\frac{1}{2}\left(\tau^{2}+1\right)
$$

2. $\widetilde{\alpha}(v)=\widehat{\alpha}(\tau(v))$, where

$$
\widehat{\alpha}(\tau)=2 \arctan (\tau)=-i \log \left(\frac{i-\tau}{i+\tau}\right) .
$$

As a consequence, we can deduce the behavior for the homoclinic close to its singularities and as $|v| \rightarrow+\infty$. Note that these singularities are in fact zeros of the function $\widetilde{r}_{\mathrm{h}}$ and therefore can be seen as collisions of the parabolic orbit of the two body problem, which occur for complex values of time.

Corollary 4.2. The rescaled homoclinic (14) with initial conditions (15) behaves as

$$
\widetilde{r}_{\mathrm{h}}(v) \sim 3 v^{2 / 3}, \quad \widetilde{y}_{\mathrm{h}}(v) \sim 2 v^{-1 / 3}, \quad \text { and } \quad \widetilde{\alpha}_{\mathrm{h}}(v)-\alpha_{0} \sim \frac{1}{3} v^{-1 / 3}
$$

as $|v| \rightarrow+\infty$.

Now we give the properties of the homoclinic close to its singularities. Note that $\tau= \pm i$ if and only if $v= \pm i / 3$. Furthermore,

$$
\tau \pm i \sim(v \pm i / 3)^{1 / 2} .
$$

Corollary 4.3. The rescaled homoclinic (14) with initial conditions (15) behaves as

$$
\widetilde{r}_{\mathrm{h}}(v) \sim C\left(v \mp \frac{i}{3}\right)^{1 / 2}, \quad \widetilde{y}_{\mathrm{h}}(v) \sim \frac{C}{2}\left(v \mp \frac{i}{3}\right)^{-1 / 2}, \quad \widetilde{\alpha}_{\mathrm{h}}(v) \sim \pm \frac{i}{2} \ln \left(v-\frac{i}{3}\right),
$$

where $C^{2}= \pm 2 i$, and

$$
e^{i \widetilde{\alpha}_{\mathrm{h}}(v)} \sim \frac{v-\frac{i}{3}}{v+\frac{i}{3}}
$$

\section{$5 \quad$ Existence of the invariant manifolds}

We devote this section to obtain parameterizations of the invariant manifolds of infinity as solutions of equation (24). Introducing the linear operator

$$
\mathcal{L}(h)=\partial_{v} h-G_{0}^{3} \partial_{\xi} h
$$

equation (24) becomes

$$
\mathcal{L}\left(T_{1}\right)=-\frac{1}{2 \widetilde{y}_{\mathrm{h}}^{2}}\left(\partial_{v} T_{1}-\frac{1}{\widetilde{r}_{\mathrm{h}}^{2}} \partial_{\xi} T_{1}\right)^{2}-\frac{1}{2 \widetilde{r}_{\mathrm{h}}^{2}}\left(\partial_{\xi} T_{1}\right)^{2}+\widehat{U}\left(v, \xi+\widetilde{\alpha}_{\mathrm{h}}\right) .
$$

where

$$
\widehat{U}(v, \theta)=V\left(\widetilde{r}_{\mathrm{h}}(v), \theta ; \mu, G_{0}\right),
$$

and $V$ is the function defined in (11). From now on, we omit the dependence on the parameters $\mu$ and $G_{0}$. To look for solutions of this equation we split the potential $\widehat{U}$ as $\widehat{U}=\widehat{U}_{0}+\widehat{U}_{1}$ where

$$
\widehat{U}_{0}(v, \theta)=-\frac{\mu}{2}\left(1-\mu-3(1-\mu) \cos ^{2} \theta\right) \frac{1}{G_{0}^{4} \widetilde{r}_{\mathrm{h}}(v)^{3}}
$$

and

$$
\widehat{U}_{1}=\widehat{U}-\widehat{U}_{0}
$$


We consider two different left inverses of $\mathcal{L}$ given by

$$
\begin{aligned}
& \mathcal{G}^{u}(h)(v, \xi)=\int_{-\infty}^{0} h\left(v+s, \xi-G_{0}^{3} s\right) d s \\
& \mathcal{G}^{s}(h)(v, \xi)=\int_{+\infty}^{0} h\left(v+s, \xi-G_{0}^{3} s\right) d s,
\end{aligned}
$$

whenever these expressions are well defined. Using these operators and the map

$$
A:(v, \xi) \mapsto(v, \xi+\widetilde{\alpha}(v)),
$$

we introduce the functions

$$
Q_{0}^{*}=\mathcal{G}^{*}\left(\widehat{U}_{0} \circ A\right), *=u, s
$$

We will see later that $Q_{0}^{*}$ are indeed well defined in suitable domains. Finally, we introduce the new unknown $Q^{*}$ satisfying

$$
T_{1}^{*}=Q_{0}^{*}+Q^{*}
$$

The equation for $Q^{*}$ is

$$
\mathcal{L}\left(Q^{*}\right)=\mathcal{F}^{*}\left(Q^{*}\right)
$$

where $\mathcal{F}^{*}$ is given by

$$
\mathcal{F}^{*}(h)=-\frac{1}{2 \widetilde{y}_{\mathrm{h}}^{2}}\left(\partial_{v} Q_{0}^{*}+\partial_{v} h-\frac{1}{\widetilde{r}_{\mathrm{h}}^{2}}\left(\partial_{\xi} Q_{0}^{*}+\partial_{\xi} h\right)\right)^{2}-\frac{1}{2 \widetilde{r}_{\mathrm{h}}^{2}}\left(\partial_{\xi} Q_{0}^{*}+\partial_{\xi} h\right)^{2}+\widehat{U}_{1}\left(v, \xi+\widetilde{\alpha}_{\mathrm{h}}(v)\right) .
$$

We devote the rest of the section to study solutions of these equations in suitable domains. First in Section 5.1 we give the main ideas of the proof and define the complex domains were we will look for the solutions of equations (41). Then, in Section 5.2 we analyze the functions $\widehat{U}_{0}$ and $\widehat{U}_{1}$. Finally, in Sections 5.3 5.5 we obtain parameterizations of the invariant manifolds in the different domains specified in Section 5.1

\subsection{Description of the proof}

The classical way to study exponentially small splitting of separatrices would be in this setting to look for the functions $Q^{u}$ and $Q^{s}$ as solutions of equations (41) in a certain complex common domain $D \times \mathbb{T}_{\sigma}$, where $D \subset \mathbb{C}$ is a domain which reaches a neighborhood of size $\mathcal{O}\left(G_{0}^{-3}\right)$ of the singularities of the unperturbed separatrix, namely $v= \pm i / 3$ and

$$
\mathbb{T}_{\sigma}=\{\xi \in \mathbb{C} /(2 \pi \mathbb{Z}):|\operatorname{Im} \xi|<\sigma\} .
$$

However, to solve equations (41) we have to face two different problems.

The first one is that equation (41) becomes singular at $v=0$ because $\widetilde{y}_{\mathrm{h}}(0)=0$ (see (15)). To overcome this problem we proceed as was done in BFGS11 (see also Gua12). We look for solutions of equations (41) in a complex domain for the variable $v$, which was called in that paper boomerang domain due to its shape. The important features of this domain is that contains both an interval of the real line of width independent of $G_{0}$ and points at a distance $\mathcal{O}\left(G_{0}^{-3}\right)$ of the singularities $v= \pm i / 3$, and that it does not contain $v=0$. This domain can be seen in Figure 5 and is defined as follows

$$
\begin{aligned}
D_{\kappa, \delta}= & \left\{v \in \mathbb{C} ;|\operatorname{Im} v|<\tan \beta_{1} \operatorname{Re} v+1 / 3-\kappa G_{0}^{-3},|\operatorname{Im} v|<-\tan \beta_{1} \operatorname{Re} v+1 / 3-\kappa G_{0}^{-3},\right. \\
& \left.|\operatorname{Im} v|>-\tan \beta_{2} \operatorname{Re} v+1 / 6-\delta\right\},
\end{aligned}
$$

where $\kappa \in(0,1 / 3), \delta \in(0,1 / 12)$ and $\beta_{1}, \beta_{2} \in(0, \pi / 2)$ are fixed independently of $G_{0}$. Therefore, this domain is non empty provided $G_{0}>1$. 


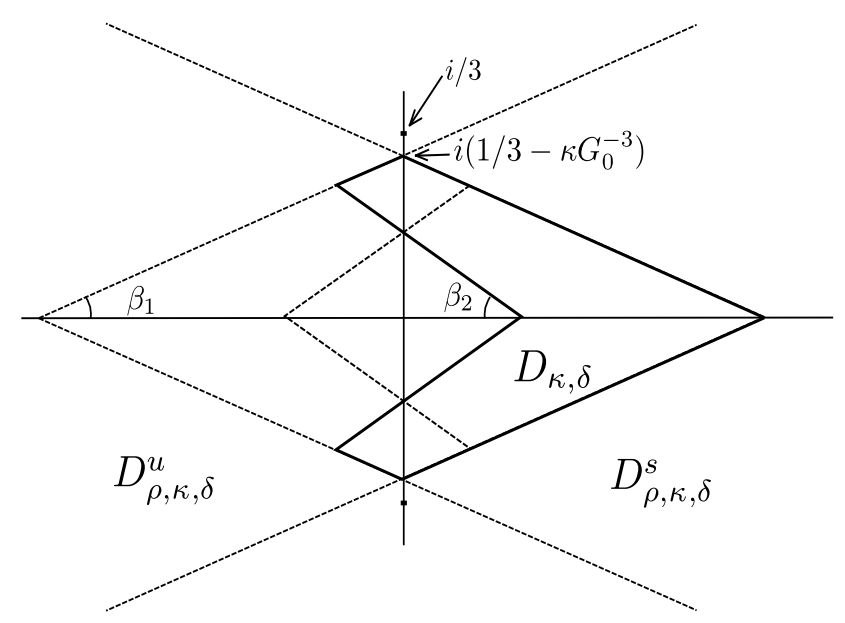

Figure 5: The domains $D_{\kappa, \delta}$ defined in (43).

To obtain the parameterizations of the invariant manifolds of infinity using equations (41), we need to impose the asymptotic conditions

$$
\begin{array}{lc}
\lim _{v \rightarrow-\infty} \widetilde{y}_{\mathrm{h}}^{-1}(v) \partial_{v} Q^{u}(v, \xi)=0, & \lim _{\operatorname{Re} v \rightarrow-\infty} \partial_{\xi} Q^{u}(v, \xi)=0 \\
\lim _{\operatorname{Re} v+\infty} \widetilde{y}_{\mathrm{h}}^{-1}(v) \partial_{v} Q^{s}(v, \xi)=0, & \lim _{v \rightarrow+\infty} \partial_{\xi} Q^{s}(v, \xi)=0 .
\end{array}
$$

However, these conditions do not have any meaning in the domain $D_{\kappa, \delta}$ since this domain is bounded. Therefore, to prove the existence of the parameterizations of the invariant manifolds in the domain $D_{\kappa, \delta}$, one has to start with different domains were these asymptotic conditions make sense and, then, one has to find a way to extend them analytically to the domain $D_{\kappa, \delta}$.

Thus, the first step is to look for solutions of equations (41) in the domains

$$
\begin{aligned}
D_{\infty, \rho}^{u} & =\{v \in \mathbb{C} ; \operatorname{Re} v<-\rho\} \\
D_{\infty, \rho}^{s} & =\{v \in \mathbb{C} ; \operatorname{Re} v>\rho\},
\end{aligned}
$$

for some $\rho>0$, where one can impose the asymptotic conditions (44). This is done in Theorem [5.5 of Section 5.3 .

To analytically extend the invariant manifolds to reach $D_{\kappa, \delta}$ we have to face the problem that these parameterizations become undefined at $v=0$. To overcome it, the second step is to first analytically extend them to the domains

$$
\begin{aligned}
D_{\rho^{\prime}, \kappa, \delta}^{u}= & \left\{v \in \mathbb{C} ;|\operatorname{Im} v|<-\tan \beta_{1} \operatorname{Re} v+1 / 3-\kappa G_{0}^{-3},|\operatorname{Im} v|>\tan \beta_{2} \operatorname{Re} v+1 / 6-\delta,\right. \\
& \left.\operatorname{Re} v>-\rho^{\prime}\right\} \\
D_{\rho^{\prime}, \kappa, \delta}^{s}= & \left\{v \in \mathbb{C} ;-v \in D_{\rho, \kappa}^{\text {out }, u}\right\},
\end{aligned}
$$

which do not contain $v=0$ (see Figure G). We choose $\rho^{\prime}>\rho$ so that $D_{\rho^{\prime}, \kappa, \delta}^{u, s} \cap D_{\infty, \rho}^{u, s} \neq \emptyset$, since then we can perform the analytic extension procedure. Note that $D_{\kappa, \delta} \subset D_{\rho, \kappa, \delta}^{s}$. This is not true for the unstable manifold. 

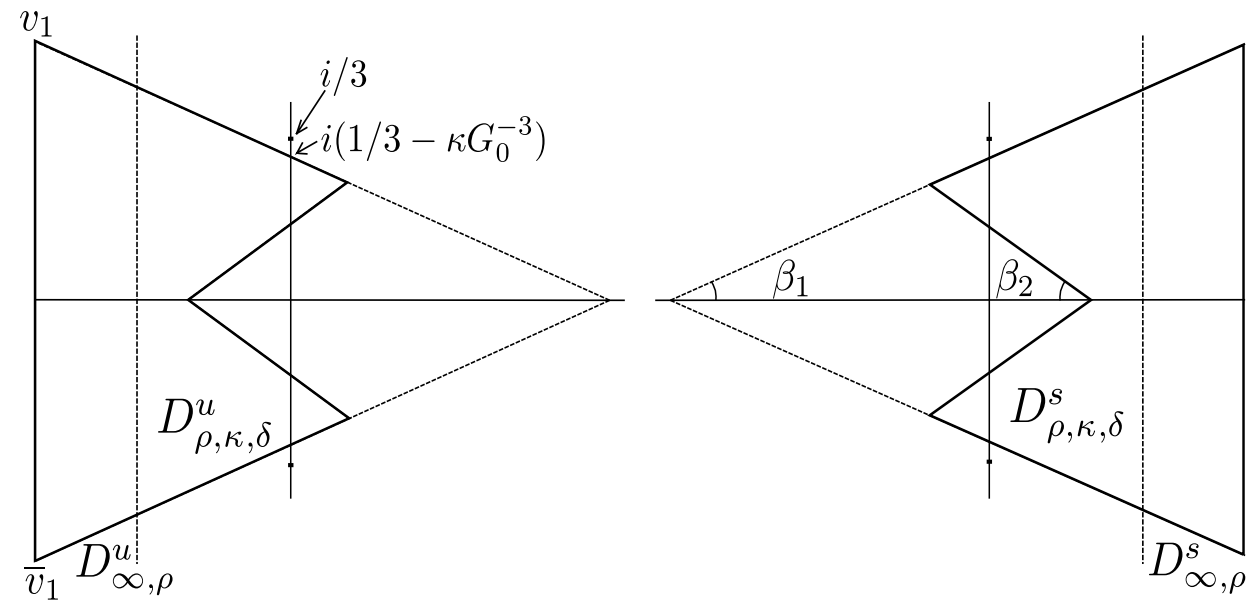

Figure 6: The domains $D_{\rho, \kappa}^{u}$ and $D_{\rho, \kappa}^{s}$ defined in (46).

When one tries to perform this analytical extension procedures, the second problem arises, which, as far as the authors know, had not appeared before in any exponentially small splitting of separatrices problem. It is well known that the solutions of (41) become very large in the upper and lower vertices of the domains $D_{\rho, \kappa, \delta}^{u, s}$, since they reach $\mathcal{O}\left(G_{0}^{-3}\right)$ neighborhoods of the singularities of the unperturbed separatrix. However, in the exponentially small splitting of separatrices problems that have been studied in the literature, using suitable weighted Banach spaces, one is able to prove that, even if they become big, they can be analytically extended to the whole domains $D_{\rho, \kappa, \delta}^{u, s}$. This is not possible for equations (41) since the solutions of these equations blow up before reaching these points. Indeed, if one looks for the analytic continuation of the functions $Q^{u, s}(v, \xi)=\sum_{\ell \in \mathbb{Z}} Q_{u, s}^{[\ell]}(v) e^{i \ell \xi}$, their Fourier coefficients $Q_{u, s}^{[\ell]}(v)$ grow exponentially in $\ell$ near the singularities $v= \pm i / 3$ and therefore one cannot obtain these analytic extensions. This technical difficulty can be overcome since to study the exponentially small splitting of separatrices phenomena one does not need to extend the functions $Q^{u, s}(v, \xi)$ but it suffices to extend their Fourier coefficients $Q_{u, s}^{[\ell]}(v)$. If the functions $Q^{u, s}$ are well defined up to neighborhoods of the singularities, these two analytic extension procedures are equivalent, but in the present paper they are not. This implies that the extension theorem that we consider in this paper, which is explained in Section 5.4 is rather different from the ones in the literature (see for instance [BFGS11]). Indeed, we will consider the Fourier coefficients of $Q^{u, s}$ as sequences of functions and we will study their extension in a Banach space of sequences of functions. This Banach space will be endowed with a weighted norm that will allow us to obtain good estimates for each Fourier coefficient. With this procedure we are able to analytically extend the Fourier coefficients of the solutions of equations (41) to the domains $D_{\rho^{\prime}, \kappa, \delta}^{u, s}$ even if the corresponding Fourier series are not convergent. This is shown in Theorem 5.10 in Section 5.4

Once the analytical extension of the Fourier coefficients of $Q^{u, s}(v, \xi)$ to $D_{\rho^{\prime}, \kappa, \delta}^{u, s}$ has been obtained, it only remains to complete the analytical extension procedure for the unstable manifold. Indeed, as we have already pointed out $D_{\kappa, \delta} \subset D_{\rho, \kappa, \delta}^{s}$ and therefore, we have the Fourier coefficients of the parameterization of the stable manifold in the boomerang domain (43), where we will study the difference between the manifolds. For the unstable manifold we still need to reach the points in $D_{\kappa, \delta}$ that do not belong to $D_{\rho, \kappa, \delta}^{u}$. That is, we need to analytically extend the Fourier coefficients of $Q^{u}$ to the domain

$$
\begin{gathered}
\widetilde{D}_{\kappa, \delta}=\left\{v \in \mathbb{C} ;|\operatorname{Im} v|<-\tan \beta_{1} \operatorname{Re} v+1 / 3-\kappa G_{0}^{-3},|\operatorname{Im} v|>-\tan \beta_{2} \operatorname{Re} v+1 / 6-\delta\right. \\
\left.|\operatorname{Im} v|<\tan \beta_{2} \operatorname{Re} v+1 / 6+\delta\right\} .
\end{gathered}
$$

(see Figure 7). 


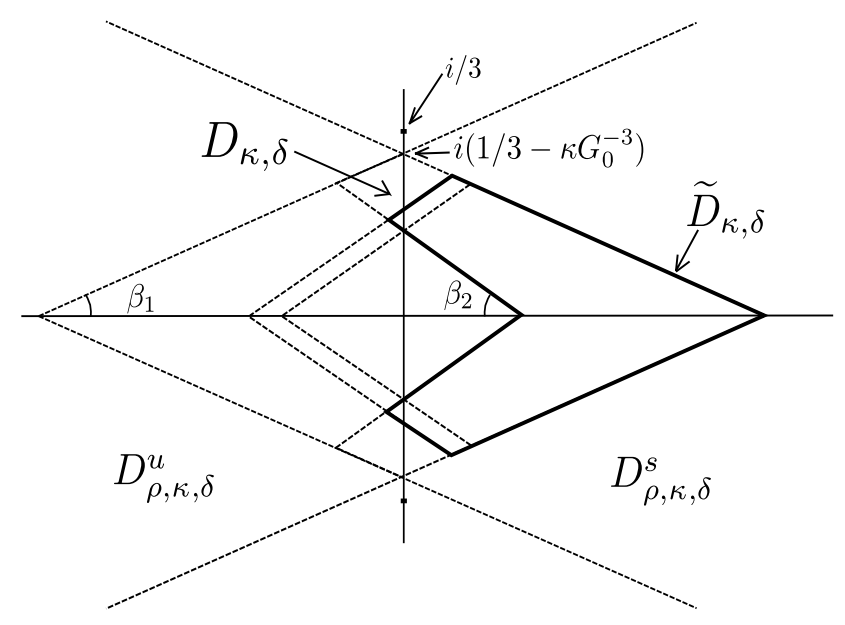

Figure 7: The domain $\widetilde{D}_{\kappa, \delta}$ defined in (47).

Indeed, it is easy to see that

$$
D_{\kappa, \delta} \subset D_{\rho, \kappa, \delta}^{u} \cup \widetilde{D}_{\kappa, \delta}
$$

To obtain the analytic extension of the Fourier coefficients of $Q^{u}$ to the domain $\widetilde{D}_{\kappa, \delta}$ we proceed as follows. First, we switch to the classical flow parameterization of the invariant manifolds DS92, which is well defined for $v=0$. This parameterization is of the form

$$
(\widetilde{r}, \widetilde{y}, \phi, \widetilde{G})=\Gamma^{u}(v, \xi)
$$

where $\Gamma^{u}=\Gamma_{0}+\Gamma_{1}^{u}, \Gamma_{0}(v, \xi)=\left(\widetilde{r}_{\mathrm{h}}(v), \widetilde{y}_{\mathrm{h}}(v), \xi+\widetilde{\alpha}_{\mathrm{h}}(v), 1\right)$ is the parameterization of the unperturbed homoclinic orbit and $\Gamma^{u}\left(v+t, \xi-G_{0}^{3} t\right)$ is a solution of the flow associated to the Hamiltonian (17).

We look for this parameterization in the domain

$$
\begin{gathered}
D_{\kappa, \delta}^{\text {flow }}=\left\{v \in \mathbb{C} ;|\operatorname{Im} v|<-\tan \beta_{1} \operatorname{Re} v+1 / 3-\kappa G_{0}^{-3},\right. \\
\left.|\operatorname{Im} v|<\tan \beta_{2} \operatorname{Re} v+1 / 6+\delta\right\},
\end{gathered}
$$

which can be seen in Figure 8 . Finally we will switch back to the original parameterization (25) in the domain $\widetilde{D}_{\kappa, \delta}=D_{\kappa, \delta}^{\text {flow }} \cap D_{\kappa, \delta}$ defined in (47), which does not contain $v=0$ and therefore equation (41) is well defined. Since the inclusion (48) is satisfied, after this procedure we will have the Fourier coefficients of the parameterization of the unstable manifold $Q^{u}$ defined in the whole domain $D_{\kappa, \delta}$. 


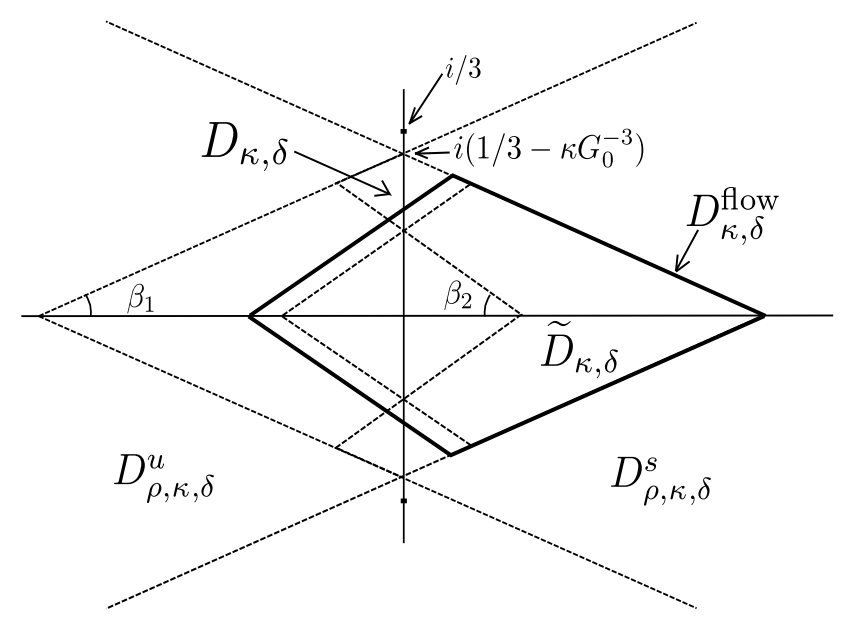

Figure 8: The domain $D_{\kappa, \delta}^{\text {flow }}$ defined in (47).

This analytical extension procedure through the flow parameterization is explained in detail in Section 5.5.1. It has three steps

1. First, we derive a parameterization of the form (49) in the domain $D_{\rho, \kappa, \delta}^{u}$ by performing a suitable change of variables to the parameterization (25), which are already known in this domain thanks to Theorem 5.10. This is done in Theorem 5.16.

2. Once we have obtained this parameterization (49) in the domain $D_{\rho, \kappa, \delta}^{u}$, we extend it, using the flow, to the whole domain $D_{\kappa, \delta}^{\text {flow }}$. This is done in Proposition 5.20

3. Finally, using again a change of variables, the last step is to switch back to the parameterization (25) in the domain $\widetilde{D}_{\kappa, \delta}=D_{\kappa, \delta}^{\text {flow }} \cap D_{\kappa, \delta}$. This is done in Proposition 5.21.

Notation. To simplify the notation, in the forthcoming sections, we will denote by $K$ any constant independent of $\mu$ and $G_{0}$ to state all the bounds.

\subsection{The perturbative potential}

We devote this section to obtain estimates for the functions $\widehat{U}_{0}$ and $\widehat{U}_{1}$ defined in (35) and (36), respectively, in the domains defined in Section 5.1] This estimate will be used in the subsequent Sections 5.35 .5

First we state the following technical lemma, whose proof is straightforward taking into account Corollary 4.3

Lemma 5.1. For any $v \in D$, where $D$ is any of the domains (43), (45), (46), (47), (50) and $G_{0}$ big enough, the following bound is satisfied

$$
\left|\frac{1}{G_{0}^{2} \widetilde{r}_{\mathrm{h}}(v)}\right| \leq \frac{K}{G_{0}^{1 / 2}}
$$

where $\widetilde{r}_{\mathrm{h}}$ is the $\widetilde{r}$ component of the homoclinic orbit (14).

Lemma 5.2. For any $(v, \theta) \in D \times \mathbb{T}$, where $D$ is any of the domains (43), (45), (46), (47), (50) and $G_{0}$ big enough, the functions $\widehat{U}_{0}$ and $\widehat{U}_{1}$ satisfy

$$
\begin{aligned}
& \left|\widehat{U}_{0}(v, \theta)\right|<\mu \frac{K}{G_{0}^{4}\left|\widetilde{r}_{\mathrm{h}}(v)\right|^{3}} \\
& \left|\widehat{U}_{1}(v, \theta)\right|<\mu \frac{K}{G_{0}^{6}\left|\widetilde{r}_{\mathrm{h}}(v)\right|^{4}} .
\end{aligned}
$$


Proof. The prove of the first bound is straightforward. For the second one it is enough to bound the remainder in Taylor's formula using Lemma 5.1 and to apply Schwarz's lemma to obtain

$$
\left|\widehat{U}(v, \theta)+\frac{1}{2} \mu\left(1-\mu-3(1-\mu) \cos ^{2} \theta\right) \frac{1}{G_{0}^{4} \widetilde{r}_{\mathrm{h}}(v)^{3}}\right| \leq \mu G_{0}^{2} \frac{K}{\left|G_{0}^{2} \widetilde{r}_{\mathrm{h}}(v)\right|^{4}} .
$$

Corollary 5.3. Denoting by $h^{[\ell]}(v)$ the $\ell$-th Fourier coefficient of a function $h(v, \theta)$, the following bounds hold

1. $\left|\widehat{U}_{0}^{[\ell]}(v)\right| \leq \mu \frac{K}{\left.G_{0}^{4}||_{\mathrm{h}}(v)\right|^{3}}$, for $|\ell|=0,2, \widehat{U}_{0}^{[\ell]}(v)=0$, for $|\ell| \neq 0,2$, and

2. $\left|\widehat{U}_{1}^{[\ell]}(v)\right| \leq \mu \frac{K}{G_{0}^{6}\left|\widetilde{r}_{\mathrm{h}}(v)\right|^{4}}$, for $\ell \in \mathbb{Z}$.

We finish this section by giving estimates for $\widetilde{\alpha}_{\mathrm{h}}(v)$, which will be crucial to extend the Fourier coefficients of the parameterization of the invariant manifolds close to the singularities of the unperturbed separatrix.

Lemma 5.4. Let $\widetilde{\alpha}_{\mathrm{h}}$ be the $\widetilde{\alpha}$ component of the homoclinic orbit (14). Let $\ell \in \mathbb{Z}$. Then, for $G_{0}>0$ big enough the function $e^{i \ell \widetilde{\alpha}_{\mathrm{h}}(v)}$ satisfies that

1. For $v \in D$, where $D$ is any of the domains (43), (46), (47), (50)

$$
\begin{aligned}
& \left|e^{i \ell \widetilde{\alpha}_{\mathrm{h}}(v)}\right| \leq\left|K \frac{v-i / 3}{v+i / 3}\right|^{|\ell| / 2} \text { for } \ell>0 \\
& \left|e^{i \ell \widetilde{\alpha}_{\mathrm{h}}(v)}\right| \leq\left|K \frac{v+i / 3}{v-i / 3}\right|^{|\ell| / 2} \text { for } \ell<0 .
\end{aligned}
$$

2. For $v \in D_{\infty, \rho}^{u, s}$ (see (45)),

$$
\left|e^{i \ell \widetilde{\alpha}_{h}(v)}\right| \leq K^{|\ell|} .
$$

Proof. The claim follows directly from the fact that, by (32),

$$
e^{i \ell \widetilde{\alpha}_{\mathrm{h}}(v(\tau))}=\left(\frac{i-\tau(v)}{i+\tau(v)}\right)^{\ell}
$$

and (33).

\subsection{The invariant manifolds close to infinity}

We devote this section to prove the existence of the invariant manifolds in the domains $D_{\infty, \rho}^{u, s} \times \mathbb{T}_{\sigma}$, where $D_{\infty, \rho}^{u, s}$ are the domains defined in (45). To this end we will look for solutions of equation (41) satisfying the asymptotic conditions (44). To obtain these solutions, we set up a fixed point argument inverting the operator $\mathcal{L}$ in (34) in suitable Banach spaces for functions defined in $D_{\infty, \rho}^{u, s} \times \mathbb{T}_{\sigma}$. We do it for the unstable manifold. The existence of the stable one is given by the symmetry property (27).

We first define norms for functions $h: D_{\infty, \rho}^{u} \rightarrow \mathbb{C}$ as

$$
\|h\|_{\nu}=\sup _{v \in D_{\infty, \rho}^{u}}\left|v^{\nu} h(v)\right| .
$$

Then, for functions $h: D_{\infty, \rho}^{u} \times \mathbb{T}_{\sigma} \rightarrow \mathbb{C}$, we define

$$
\|h\|_{\nu, \sigma}=\sum_{\ell \in \mathbb{Z}}\left\|h^{[\ell]}\right\|_{\nu} e^{|\ell| \sigma}
$$


and the functional space

$$
\mathcal{Z}_{\nu, \rho, \sigma}=\left\{h: D_{\infty, \rho}^{u} \times \mathbb{T}_{\sigma} \rightarrow \mathbb{C}: \text { real-analytic, }\|h\|_{\nu, \sigma}<\infty\right\} .
$$

It can be checked that it is a Banach space for any fixed $\nu \geq 0$. Moreover, since equation (41) involves both $Q$ and its derivatives $\partial_{v} Q$ and $\partial_{\xi} Q$ we will need to deal with Banach spaces which control at the same time the norms of a function and its derivatives. To this end we define the norm

$$
\llbracket h\left\|_{\nu, \sigma}=\right\| h\left\|_{\nu, \sigma}+\right\| \partial_{v} h\left\|_{\nu+1, \sigma}+G_{0}^{3}\right\| \partial_{\xi} h \|_{\nu+1, \sigma},
$$

and the corresponding Banach space

$$
\widetilde{\mathcal{Z}}_{\nu, \rho, \sigma}=\left\{h: D_{\infty, \rho}^{u} \times \mathbb{T}_{\sigma} \rightarrow \mathbb{C}: \text { real-analytic, }\left\lfloor h \rrbracket_{\nu, \sigma}<\infty\right\} .\right.
$$

Note that

$$
\widetilde{\mathcal{Z}}_{\nu, \rho, \sigma} \subset \mathcal{Z}_{\nu, \rho, \sigma} .
$$

Once we have defined the suitable Banach spaces, we can state the main theorem of this section.

Theorem 5.5. Fix constants $\rho_{0}>0$ and $\sigma_{0}>0$. Then, for $G_{0}>0$ big enough, there exists a unique solution $Q^{u}$ of equation (41) in $\widetilde{\mathcal{Z}}_{5 / 3, \rho_{0}, \sigma_{0}}$ satisfying (44). Moreover, it satisfies

$$
\llbracket Q^{u} \Perp_{5 / 3, \sigma_{0}} \leq b_{0} \mu G_{0}^{-6},
$$

for a constant $b_{0}>0$ independent of $\mu$ and $G_{0}$.

Furthermore, if we define the function

$$
L_{1}^{u}(v, \xi)=\int_{-\infty}^{0} \widehat{U}_{1}\left(v+s, \xi-G_{0}^{3} s+\widetilde{\alpha}_{\mathrm{h}}(v+s)\right) d s,
$$

where $\widetilde{\alpha}_{\mathrm{h}}$ and $\widehat{U}_{1}$ are defined in (14) and (36), respectively, we have that $L_{1}^{u} \in \widetilde{\mathcal{Z}}_{5 / 3, \rho_{0}, \sigma_{0}}$, satisfies

$$
\left\|L_{1}^{u}\right\|_{5 / 3, \sigma_{0}} \leq K \mu G_{0}^{-6}
$$

and

$$
\left\|Q^{u}-L_{1}^{u}\right\|_{5 / 3, \sigma_{0}} \leq K \mu^{2} G_{0}^{-8} .
$$

The first step to prove this theorem is to invert operator $\mathcal{L}$ in equation (41) to set up a fixed point argument for $Q^{u}$. To this end we consider the operator $\mathcal{G}=\mathcal{G}^{u}$ defined in (37). Since we are only dealing with the proof of existence of the parameterization of the unstable manifold, from now on, we omit the superindex $u$ wherever there is no risk of confusion.

Next lemma, whose proof is analogous to the one of Lemma 5.5 of GOS10, gives properties about how the operator $\mathcal{G}$ acts on the Banach spaces $\mathcal{Z}_{\nu, \rho, \sigma}$.

Lemma 5.6. The operator $\mathcal{G}=\mathcal{G}^{u}$, defined in (37), when considered acting on the spaces $\mathcal{Z}_{\nu, \rho, \sigma}$ satisfies the following properties.

1. For any $\nu>1, \mathcal{G}: \mathcal{Z}_{\nu, \rho, \sigma} \rightarrow \mathcal{Z}_{\nu-1, \rho, \sigma}$ is well defined and linear continuous. Moreover, $\mathcal{L} \circ \mathcal{G}=\mathrm{Id}$.

2. If $h \in \mathcal{Z}_{\nu, \rho, \sigma}$ for some $\nu>1$, then

$$
\|\mathcal{G}(h)\|_{\nu-1, \rho, \sigma} \leq K\|h\|_{\nu, \sigma} .
$$

3. If $h \in \mathcal{Z}_{\nu, \rho, \sigma}$ for some $\nu \geq 1$, then $\partial_{v} \mathcal{G}(h) \in \mathcal{Z}_{\nu, \rho, \sigma}$ and

$$
\left\|\partial_{v} \mathcal{G}(h)\right\|_{\nu, \sigma} \leq K\|h\|_{\nu, \sigma} .
$$


4. If $h \in \mathcal{Z}_{\nu, \rho, \sigma}$ for some $\nu \geq 1$, then $\partial_{\xi} \mathcal{G}(h) \in \mathcal{Z}_{\nu, \rho, \sigma}$ and

$$
\left\|\partial_{\xi} \mathcal{G}(h)\right\|_{\nu, \sigma} \leq K G_{0}^{-3}\|h\|_{\nu, \sigma} .
$$

5. From the previous three statements, one can conclude that if $h \in \mathcal{Z}_{\nu, \rho, \sigma}$ for some $\nu>1$, then $\mathcal{G}(h) \in \widetilde{\mathcal{Z}}_{\nu-1, \rho, \sigma}$ and

$$
\llbracket \mathcal{G}(h) \rrbracket_{\nu-1, \sigma} \leq K\|h\|_{\nu, \sigma} .
$$

We look for a fixed point of the operator

$$
\widetilde{\mathcal{F}}=\mathcal{G} \circ \mathcal{F}
$$

where $\mathcal{G}$ and $\mathcal{F}$ are the operators defined in (37) and (42) respectively, in the space $\widetilde{\mathcal{Z}}_{5 / 3, \rho_{0}, \sigma_{0}}$ defined in (51). Theorem [5.5] is a straightforward consequence of the following proposition.

Proposition 5.7. Let us fix $\rho_{0}>0$ and $\sigma_{0}>0$. Then, if $G_{0}$ is big enough there exists a constant $b_{0}>0$ such that the operator $\widetilde{\mathcal{F}}$ in (55) has a fixed point $Q^{u} \in B\left(b_{0} \mu G_{0}^{-6}\right) \subset \widetilde{\mathcal{Z}}_{5 / 3, \rho_{0}, \sigma_{0}}$. Moreover, the function $L_{1}^{u}$ defined in (52) satisfies (53) and $Q^{u}$ satisfies (54).

Before proving this proposition, we state a technical lemma which gives properties of the functions $Q_{0}$ and $\widehat{U}_{1}$ defined in (39) and (35).

Lemma 5.8. The functions $Q_{0}$ and $\widehat{U}_{1}$ satisfy that $Q_{0} \in \widetilde{\mathcal{Z}}_{1, \rho_{0}, \sigma_{0}}$ and $\widehat{U}_{1} \circ A \in \mathcal{Z}_{8 / 3, \rho_{0}, \sigma_{0}}$, where $A$ is the map defined in (38). Moreover,

$$
\begin{aligned}
\| Q_{0} \rrbracket_{1, \sigma_{0}} & \leq K \mu G_{0}^{-4} \\
\left\|\widehat{U}_{1} \circ A\right\|_{8 / 3, \sigma_{0}} & \leq K \mu G_{0}^{-6} .
\end{aligned}
$$

Proof. For the statements referred to $Q_{0}$, it is enough to use the bound for $\widehat{U}_{0}$ in Lemma 5.2 and Lemma [5.6. The statements for $\widehat{U}_{1} \circ A$ follow from Corollary 5.3 and Lemma 5.4.

Proof of Proposition 5.7. First, we see that $\widetilde{\mathcal{F}}=\mathcal{G} \circ \mathcal{F}$ is well defined from $\widetilde{\mathcal{Z}}_{5 / 3, \rho_{0}, \sigma_{0}}$ to itself. Indeed if $h \in$ $\widetilde{\mathcal{Z}}_{5 / 3, \rho_{0}, \sigma_{0}}$, using Lemma 5.8 and the properties of the separatrix parameterization given in Corollary 4.2 one can easily see that $\mathcal{F}(h) \in \mathcal{Z}_{8 / 3, \rho_{0}, \sigma_{0}}$. Therefore, using the last statement of Lemma 5.6, we obtain that $\mathcal{G} \circ \mathcal{F}(h) \in \widetilde{\mathcal{Z}}_{5 / 3, \rho_{0}, \sigma_{0}}$.

Now we bound $\widetilde{\mathcal{F}}(0)=\mathcal{G} \circ \mathcal{F}(0)$. By the definition of $\mathcal{F}$ in (42), we have that

$$
\mathcal{F}(0)=\frac{1}{2 \widetilde{y}_{\mathrm{h}}^{2}}\left(\partial_{v} Q_{0}-\frac{1}{\widetilde{r}_{\mathrm{h}}^{2}} \partial_{\xi} Q_{0}\right)^{2}-\frac{1}{2 \widetilde{r}_{\mathrm{h}}^{2}}\left(\partial_{\xi} Q_{0}\right)^{2}+\widehat{U}_{1} \circ A
$$

Thus, applying Lemma 5.8 and Corollary 4.2 , we have that $\mathcal{F}(0) \in \mathcal{Z}_{8 / 3, \rho_{0}, \sigma_{0}}$ and satisfies $\|\mathcal{F}(0)\|_{8 / 3, \sigma_{0}} \leq$ $K \mu G_{0}^{-6}$. Then, applying the last statement of Lemma 5.6, we obtain that there exists a constant $b_{0}>0$ such that

$$
\llbracket \widetilde{\mathcal{F}}(0) \rrbracket_{5 / 3, \sigma_{0}} \leq \frac{b_{0}}{2} \mu G_{0}^{-6} .
$$

Now we have to prove that the operator $\widetilde{\mathcal{F}}$ is contractive in $B\left(b_{0} \mu G_{0}^{-6}\right) \subset \widetilde{\mathcal{Z}}_{5 / 3, \rho_{0}, \sigma_{0}}$. Take $h_{1}, h_{2} \in$ $B\left(b_{0} \mu G_{0}^{-6}\right) \subset \widetilde{\mathcal{Z}}_{5 / 3, \rho_{0}, \sigma_{0}}$. Using the last statement of Lemma 5.6 we have that

$$
\llbracket \widetilde{\mathcal{F}}\left(h_{2}\right)-\widetilde{\mathcal{F}}\left(h_{1}\right) \bigsqcup_{5 / 3, \sigma_{0}} \leq K\left\|\mathcal{F}\left(h_{2}\right)-\mathcal{F}\left(h_{1}\right)\right\|_{8 / 3, \sigma_{0}} .
$$

Thus, we only need to bound the right hand side of this formula. To this end, we write it as

$$
\begin{aligned}
\mathcal{F}\left(h_{2}\right)-\mathcal{F}\left(h_{1}\right)= & \frac{1}{2 \widetilde{y}_{\mathrm{h}}^{2}}\left(\left(2 \partial_{v} Q_{0}+\partial_{v} h_{1}+\partial_{v} h_{2}\right)-\frac{1}{\widetilde{r}_{\mathrm{h}}^{2}}\left(2 \partial_{\xi} Q_{0}+\partial_{\xi} h_{1}+\partial_{\xi} h_{2}\right)\right)\left(\partial_{v} h_{2}-\partial_{v} h_{1}\right) \\
& -\frac{1}{2 \widetilde{y}_{\mathrm{h}}^{2} \widetilde{r}^{2}}\left(\left(2 \partial_{v} Q_{0}+\partial_{v} h_{1}+\partial_{v} h_{2}\right)-\frac{1}{\widetilde{r}_{\mathrm{h}}^{2}}\left(2 \partial_{\xi} Q_{0}+\partial_{\xi} h_{1}+\partial_{\xi} h_{2}\right)\right)\left(\partial_{\xi} h_{2}-\partial_{\xi} h_{1}\right) \\
& +\frac{1}{2 \widetilde{r}_{\mathrm{h}}^{2}}\left(2 \partial_{\xi} Q_{0}+\partial_{\xi} h_{1}+\partial_{\xi} h_{2}\right)\left(\partial_{\xi} h_{2}-\partial_{\xi} h_{1}\right) .
\end{aligned}
$$


Using the bounds for $Q_{0}$ obtained in Lemma 5.8 , Corollary 4.2 and that $h_{1}, h_{2} \in B\left(b_{0} \mu G_{0}^{-6}\right) \subset \widetilde{\mathcal{Z}}_{5 / 3, \rho_{0}, \sigma_{0}}$, we obtain that

$$
\left\|\mathcal{F}\left(h_{2}\right)-\mathcal{F}\left(h_{1}\right)\right\|_{8 / 3, \sigma_{0}} \leq K \mu G_{0}^{-4} \llbracket h_{2}-h_{1} \rrbracket_{5 / 3, \sigma_{0}} .
$$

Then, since $h_{1}, h_{2} \in \widetilde{\mathcal{Z}}_{5 / 3, \rho_{0}, \sigma_{0}}$, we can conclude that

$$
\llbracket \widetilde{\mathcal{F}}\left(h_{2}\right)-\tilde{\mathcal{F}}\left(h_{1}\right) \rrbracket_{5 / 3, \sigma_{0}} \leq K\left\|\mathcal{F}\left(h_{2}\right)-\mathcal{F}\left(h_{1}\right)\right\|_{8 / 3, \sigma_{0}} \leq K \mu G_{0}^{-4} \llbracket h_{2}-h_{1} \rrbracket_{5 / 3, \sigma_{0}},
$$

and therefore, taking $G_{0}$ big enough, it is contractive and has a unique fixed point in $B\left(b_{0} \mu G_{0}^{-6}\right) \subset$ $\widetilde{\mathcal{Z}}_{5 / 3, \rho_{0}, \sigma_{0}}$. This completes the proof of the first claim.

Since $L_{1}^{u}=\mathcal{G}\left(\widehat{U}_{1} \circ A\right)$, inequality (53) follows from Lemma 5.8 and the second statement of Lemma 5.6

Finally, to prove inequality (54) we note that, since $Q^{u}$ is a fixed point of $\widetilde{\mathcal{F}}$,

$$
\left\|Q^{u}-L_{1}^{u}\right\|_{5 / 3, \sigma_{0}} \leq\left\|\widetilde{\mathcal{F}}\left(Q^{u}\right)-\widetilde{\mathcal{F}}(0)\right\|_{5 / 3, \sigma_{0}}+\left\|\widetilde{\mathcal{F}}(0)-L_{1}^{u}\right\|_{5 / 3, \sigma_{0}} .
$$

The first term in the right hand side can be bounded using that $\widetilde{\mathcal{F}}$ is Lipschitz,

$$
\left\|\widetilde{\mathcal{F}}\left(Q^{u}\right)-\widetilde{\mathcal{F}}(0)\right\|_{5 / 3, \sigma_{0}} \leq \llbracket \widetilde{\mathcal{F}}\left(Q^{u}\right)-\widetilde{\mathcal{F}}(0) \rrbracket_{5 / 3, \sigma_{0}} \leq K \mu G_{0}^{-4} \llbracket Q^{u} \rrbracket_{5 / 3, \sigma_{0}} \leq K \mu^{2} G_{0}^{-10} .
$$

To obtain a bound of the second term in the right hand side of (58) we first note that, by (56) and Lemma 5.8 .

$$
\left\|\mathcal{F}(0)-\widehat{U}_{1} \circ A\right\|_{8 / 3, \sigma_{0}}=\left\|\frac{1}{2 \widetilde{y}_{\mathrm{h}}^{2}}\left(\partial_{v} Q_{0}-\frac{1}{\widehat{r}_{\mathrm{h}}^{2}} \partial_{\xi} Q_{0}\right)^{2}+\frac{1}{2 \widetilde{r}_{\mathrm{h}}^{2}} \partial_{\xi} Q_{0}^{2}\right\|_{8 / 3, \sigma_{0}} \leq K^{2} \mu^{2} G_{0}^{-8} .
$$

The claim follows then applying the second statement of Lemma [5.6 to $\widetilde{\mathcal{F}}(0)-L_{1}^{u}=\mathcal{G}\left(\mathcal{F}(0)-\widehat{U}_{1} \circ A\right)$.

\subsection{The invariant manifolds close to the singularities}

As we have explained in Section 5.1 once one has obtained the existence of the invariant manifolds close to infinity, the usual next step when one wants to study the exponentially small splitting of separatrices phenomena is to extend the parameterizations of the invariant manifolds to the domains $D_{\rho, \kappa, \delta}^{u, s}$ defined in (46). That is, up to points at distance $\mathcal{O}\left(G_{0}^{-3}\right)$ of the singularities of the separatrix (14) at $v= \pm i / 3$. This is not possible in this problem since the parameterizations of the invariant manifolds blow up before reaching these points. Indeed, if one looks at the analytic continuation of the function $L_{1}^{u}(v, \xi)=$ $\sum_{\ell \in \mathbb{Z}} L_{1}^{[\ell]}(v) e^{i k \xi}$ defined in (52), which is the first order approximation of the function $Q^{u}(v, \xi)$ obtained in Proposition 5.7, one can see that its Fourier coefficients $L_{1}^{[\ell]}$ grow exponentially in $\ell$. Therefore, one cannot obtain this analytic extension and neither the one of $Q^{u}$. We overcome this difficulty analytically extending the Fourier coefficients $Q^{[\ell]}$ instead of the function $Q^{u}$. It turns out that this is sufficient to study the exponentially small splitting of separatrices. To this end, we consider the Fourier coefficients of $Q^{u}$ as a sequence of functions and we study their extension in a Banach space of sequence of functions. We endow the Banach space with a weighted norm that allows us to obtain good estimates for each Fourier coefficient.

The structure of this section goes as follows. First in Section 5.4.1 we define the Banach spaces for the Fourier coefficients and their sequences. We also show that these spaces have an algebra-like structure with respect to the classical product of Fourier series. Note that the "Fourier series" we are dealing with are formal and therefore this algebra structure is not obvious. Then, in Section 5.4 .2 we set up a fixed point argument and prove the existence of the Fourier coefficients of the parameterization of the invariant manifolds in the domains $D_{\rho, \kappa, \delta}^{u, s}$. We deal only with the unstable manifold since the existence of the stable one is given by the symmetry (27). 


\subsubsection{Weighted Fourier norms and Banach spaces}

We devote this section to study the weighted Fourier norms. To this end we first define norms for the Fourier coefficients, that is, for functions $h: D_{\rho, \kappa, \delta}^{u} \rightarrow \mathbb{C}$. We define

$$
\|h\|_{\nu_{-}, \nu_{+}}=\sup _{v \in D_{\rho, \kappa, \delta}^{u}}\left|(v-i / 3)^{\nu_{+}}(v+i / 3)^{\nu_{-}} h(v)\right|
$$

and the corresponding Banach space

$$
\mathcal{X}_{\nu_{-}, \nu_{+}, \rho, \kappa, \delta}=\left\{h: D_{\rho, \kappa, \delta}^{u} \rightarrow \mathbb{C}: \text { analytic, }\|h\|_{\nu_{-}, \nu_{+}}<\infty\right\} .
$$

Next step is to define a weighted norm for sequences of functions. To denote a sequence $\left\{h^{[\ell]}\right\}_{\ell \in \mathbb{Z}}$ we keep the Fourier series notation

$$
h(v, \xi)=\sum_{\ell \in \mathbb{Z}} h^{[\ell]}(v) e^{i \ell \xi}
$$

but we want to stress that this series is a formal series and that $h(v, \xi)$ is not necessarily a function. That is, each Fourier coefficient is an analytic function defined in $D_{\rho, \kappa, \delta}^{u}$ but its sum does not need to be convergent for any $\xi \in \mathbb{T}_{\sigma}$. We define the following norm for these sequences of Fourier coefficient: 1$]$

$$
\|h\|_{\nu, \sigma}=\sum_{\ell \in \mathbb{Z}}\left\|h^{[\ell]}\right\|_{\nu+\ell / 2, \nu-\ell / 2} e^{|\ell| \sigma}
$$

and the corresponding Banach space

$$
\mathcal{Y}_{\nu, \rho, \kappa, \delta, \sigma}=\left\{h(v, \xi)=\sum_{\ell \in \mathbb{Z}} h^{[\ell]}(v) e^{i \ell \xi}: h^{[\ell]} \in \mathcal{X}_{\nu+\ell / 2, \nu-\ell / 2, \rho, \kappa, \delta},\|h\|_{\nu, \kappa, \sigma}<\infty\right\} .
$$

Next lemma gives algebra properties for these Banach spaces. Its proof is straightforward.

Lemma 5.9. The spaces $\mathcal{Y}_{\nu, \rho, \kappa, \delta, \sigma}$ satisfy the following properties:

- If $h \in \mathcal{Y}_{\nu, \rho, \kappa, \delta, \sigma}$ and $g \in \mathcal{Y}_{\eta, \rho, \kappa, \delta, \sigma}$, then the formal product of Fourier series hy defined as usual by

$$
(h g)^{[\ell]}(v)=\sum_{k \in \mathbb{Z}} h^{[k]} g^{[\ell-k]}
$$

satisfies that $h g \in \mathcal{Y}_{\nu+\eta, \rho, \kappa, \delta, \sigma}$ and

$$
\|h g\|_{\nu+\eta, \sigma} \leq\|h\|_{\nu, \sigma}\|g\|_{\eta, \sigma} .
$$

- If $h \in \mathcal{Y}_{\nu, \rho, \kappa, \delta, \sigma}$, then $h \in \mathcal{Y}_{\nu+\eta, \rho, \kappa, \delta, \sigma}$ with $\eta>0$ and

$$
\|h\|_{\nu+\eta, \sigma} \leq K\|h\|_{\nu, \sigma} .
$$

- If $h \in \mathcal{Y}_{\nu, \rho, \kappa, \delta, \sigma}$, then $h \in \mathcal{Y}_{\nu-\eta, \rho, \kappa, \delta, \sigma}$ with $\eta>0$ and

$$
\|h\|_{\nu-\eta, \sigma} \leq K G_{0}^{3 \eta}\|h\|_{\nu, \sigma} .
$$

\footnotetext{
${ }^{1}$ An equivalent norm is defined by

$$
\|h\|_{\nu, \sigma}=\sum_{\ell \in \mathbb{Z}}\left\|h^{[\ell]} e^{-i \ell \widetilde{\alpha}_{h}}\right\|_{\nu} e^{|\ell| \sigma}, \quad \text { where } \quad\|h\|_{\nu}=\sup _{v \in D_{\rho, \kappa, \delta}^{u}}\left|(v-i / 3)^{\nu}(v+i / 3)^{\nu} h(v)\right|
$$
}

and $\widetilde{\alpha}_{\mathrm{h}}$ has been defined in (14). 
In Section 5.3. we needed to control at the same time the size of functions $h \in \mathcal{Z}_{\nu, \rho, \sigma}$ and the size of their derivatives $\partial_{v} h$ and $\partial_{\xi} h$. This fact has prompted us to deal with the Banach spaces (51). To perform the Extension Theorem 5.10 we will also need to control the size of the derivatives for sequences of Fourier coefficients $h \in \mathcal{Y}_{\nu, \rho, \kappa, \delta, \sigma}$. The derivatives of sequences are defined in the natural way

$$
\begin{aligned}
& \partial_{v} h(v, \xi)=\sum_{\ell \in \mathbb{Z}} \partial_{v} h^{[\ell]}(v) e^{i \ell \xi}, \\
& \partial_{\xi} h(v, \xi)=\sum_{\ell \in \mathbb{Z}}(i \ell) h^{[\ell]}(v) e^{i \ell \xi} .
\end{aligned}
$$

Thus, we define the Banach space of formal Fourier series

$$
\widetilde{\mathcal{Y}}_{\nu, \rho, \kappa, \delta, \sigma}=\left\{h(v, \xi)=\sum_{\ell \in \mathbb{Z}} h^{[\ell]}(v) e^{i \ell \xi}: h^{[\ell]} \in \mathcal{X}_{\nu+\ell / 2, \nu-\ell / 2, \rho, \kappa, \delta},\|h\|_{\nu, \sigma}<\infty\right\},
$$

where

$$
\|h\|_{\nu, \sigma}=\|h\|_{\nu, \sigma}+\left\|\partial_{v} h\right\|_{\nu+1, \sigma}+G_{0}^{3}\left\|\partial_{\xi} h\right\|_{\nu+1, \sigma}
$$

\subsubsection{The fixed point argument}

In Theorem 5.5 we have obtained the existence of the function $Q^{u}$ in the domain $D_{\infty, \rho_{0}}^{u} \times \mathbb{T}_{\sigma_{0}}$ and, therefore, we have obtained the existence of its Fourier coefficients $Q^{[\ell]}$ in the domain $D_{\infty, \rho_{0}}^{u}$. We devote this section to obtain the analytic continuation of these Fourier coefficients to the domain $D_{\rho_{1}, \kappa, \delta}^{u}$ defined in (46), where we choose $\rho_{1}>\rho_{0}$ so that the domains $D_{\infty, \rho_{0}}^{u}$ and $D_{\rho_{1}, \kappa, \delta}^{u}$ overlap. The main theorem of this section is the following.

Theorem 5.10. Consider $\rho_{0}$ and $\sigma_{0}$ the constants given by Theorem [5.5, $\rho_{1}>\rho_{0}, \kappa_{0}, \delta_{0}>0$ and the function $Q^{u}$ obtained in Theorem 5.5. Then, for $G_{0}$ big enough, its Fourier coefficients can be analytically extended to $D_{\rho_{1}, \kappa_{0}, \delta_{0}}^{u}$ and the sequence given by the Fourier series

$$
Q^{u}(v, \xi)=\sum_{\ell \in \mathbb{Z}} Q^{[\ell]}(v) e^{i \ell \xi}
$$

satisfies that $Q^{u} \in \widetilde{\mathcal{Y}}_{1, \rho_{1}, \kappa_{0}, \delta_{0}, \sigma_{0}}$ and

$$
\llbracket Q^{u} \Perp_{1, \sigma_{0}} \leq b_{1} \mu G_{0}^{-6}
$$

for a constant $b_{1}>0$ independent of $\mu$ and $G_{0}$.

Moreover, the Fourier coefficients of the function $L_{1}^{u}$ defined in (52) can be analytically extended to $D_{\rho_{1}, \kappa_{0}, \delta_{0}}^{u}$ and its sequence belongs to $\mathcal{Y}_{1, \rho_{1}, \kappa_{0}, \delta_{0}, \sigma_{0}}$. Furthermore, it satisfies

$$
\left\|L_{1}^{u}\right\|_{1, \sigma_{0}} \leq K \mu G_{0}^{-6}
$$

and

$$
\left\|Q^{u}-L_{1}^{u}\right\|_{1, \sigma_{0}} \leq K \mu^{2} G_{0}^{-8}
$$

We devote the rest of the section to prove this theorem. To this end, we derive a fixed point argument from equation (41). We follow the approach considered in BFGS11. We consider an operator $\widetilde{\mathcal{G}}$ which acts on $\mathcal{Y}_{\nu, \rho, \kappa, \delta, \sigma}$ and is a left inverse of the operator $\mathcal{L}$ in (34). $\widetilde{\mathcal{G}}$ is defined acting on the Fourier coefficients. We consider $v_{1}, \bar{v}_{1} \in \mathbb{C}$ the vertices of the domain $D_{\rho, \kappa, \delta}^{u}$ (see Figure 6, the bar denotes the complex conjugate). Then, we define $\widetilde{\mathcal{G}}$ as

$$
\widetilde{\mathcal{G}}(h)(v, \xi)=\sum_{\ell \in \mathbb{Z}} \widetilde{\mathcal{G}}(h)^{[\ell]}(v) e^{i \ell \xi}
$$


where its Fourier coefficients are given by

$$
\begin{array}{ll}
\widetilde{\mathcal{G}}(h)^{[\ell]}(v)=\int_{v_{1}}^{v} e^{i \ell G_{0}^{3}(v-t)} h^{[\ell]}(t) d t & \text { for } \ell<0 \\
\widetilde{\mathcal{G}}(h)^{[0]}(v)=\int_{-\rho}^{v} h^{[0]}(t) d t & \\
\widetilde{\mathcal{G}}(h)^{[\ell]}(v)=\int_{\bar{v}_{1}}^{v} e^{i \ell G_{0}^{3}(v-t)} h^{[\ell]}(t) d t & \text { for } \ell>0 .
\end{array}
$$

Observe that the definition of the operator $\widetilde{\mathcal{G}}$ depends on the domain, since it involves the vertices $v_{1}$, $\bar{v}_{1}$ and also $\rho$. Next lemma, whose proof is analogous to Lemma 5.5 in GOS10, gives properties of this operator acting on the space $\mathcal{Y}_{\nu, \rho, \kappa, \delta, \sigma}$.

Lemma 5.11. The operator $\widetilde{\mathcal{G}}$ in (66) satisfies the following properties.

1. If $h \in \mathcal{Y}_{\nu, \rho, \kappa, \delta, \sigma}$ for some $\nu \geq 0$, then $\widetilde{\mathcal{G}}(h) \in \mathcal{Y}_{\nu, \rho, \kappa, \delta, \sigma}, \mathcal{L} \circ \widetilde{\mathcal{G}}(h)=h$ and

$$
\|\widetilde{\mathcal{G}}(h)\|_{\nu, \sigma} \leq K\|\widetilde{h}\|_{\nu, \sigma}
$$

2. If $h \in \mathcal{Y}_{\nu, \rho, \kappa, \delta, \sigma}$ for some $\nu>1$, then $\widetilde{\mathcal{G}}(h) \in \mathcal{Y}_{\nu-1, \rho, \kappa, \delta, \sigma}$ and

$$
\|\widetilde{\mathcal{G}}(h)\|_{\nu-1, \sigma} \leq K\|h\|_{\nu, \sigma} .
$$

3. If $h \in \mathcal{Y}_{\nu, \rho, \kappa, \delta, \sigma}$ for some $\nu \geq 1$, then $\partial_{v} \widetilde{\mathcal{G}}(h) \in \mathcal{Y}_{\nu, \rho, \kappa, \delta, \sigma}$ and

$$
\left\|\partial_{v} \widetilde{\mathcal{G}}(h)\right\|_{\nu, \sigma} \leq K\|h\|_{\nu, \sigma}
$$

4. If $h \in \mathcal{Y}_{\nu, \rho, \kappa, \delta, \sigma}$ for some $\nu \geq 1$, then $\partial_{\xi} \widetilde{\mathcal{G}}(h) \in \mathcal{Y}_{\nu, \rho, \kappa, \delta, \sigma}$ and

$$
\left\|\partial_{\xi} \widetilde{\mathcal{G}}(h)\right\|_{\nu, \sigma} \leq K G_{0}^{-3}\|h\|_{\nu, \sigma}
$$

5. From the previous three statements, one can conclude that if $h \in \mathcal{Y}_{\nu, \rho, \kappa, \delta, \sigma}$ for some $\nu>1$, then $\widetilde{\mathcal{G}}(h) \in \widetilde{\mathcal{Y}}_{\nu-1, \rho, \kappa, \delta, \sigma}$ and

$$
\llbracket \widetilde{\mathcal{G}}(h)\left\|_{\nu-1, \sigma} \leq K\right\| h \|_{\nu, \sigma} .
$$

We use the operator $\widetilde{\mathcal{G}}$ to set up a fixed point argument from equation (41). Nevertheless, since we want to obtain the analytic continuation of the Fourier coefficients obtained in Theorem 5.5, we impose certain initial conditions. Since the operator $\widetilde{\mathcal{G}}$ involves integration from different initial points depending on the harmonic, we impose different initial conditions for each harmonic. We define

$$
F(v, \xi)=\sum_{\ell<0} e^{i \ell G_{0}^{3}\left(v-v_{1}\right)} Q^{[\ell]}\left(v_{1}\right) e^{i \ell \xi}+Q^{[0]}\left(-\rho_{1}\right)+\sum_{\ell>0} e^{i \ell G_{0}^{3}\left(v-\bar{v}_{1}\right)} Q^{[\ell]}\left(\bar{v}_{1}\right) e^{i \ell \xi}
$$

Recall that, since $\rho_{1}>\rho_{0}, v_{1}, \bar{v}_{1},-\rho_{1} \in D_{\infty, \rho_{0}}^{u}$ and therefore, by Theorem 5.5, $F$ is already known. Moreover, it is straightforward to see that $F \in \widetilde{\mathcal{Y}}_{0, \rho_{1}, \kappa_{0}, \delta_{0}, \sigma_{0}}$ and

$$
\llbracket F \rrbracket_{0, \sigma_{0}} \leq K \mu G_{0}^{-6} .
$$

With this prescribed initial condition, it can be seen that the solutions of equation

$$
Q(v, \xi)=F(v, \xi)+\widetilde{\mathcal{G}} \circ \mathcal{F}(Q)(v, \xi),
$$


understood as an equation of (not necessarily convergent) Fourier series with analytic coefficients, give the analytic continuation of the Fourier coefficients $Q^{[\ell]}(v)$ obtained in Proposition 5.7. Note that it is not obvious that this equation is well defined for $Q \in \widetilde{\mathcal{Y}}_{\nu, \rho_{1}, \kappa_{0}, \delta_{0}, \sigma_{0}}$ for any $\nu>0$. Nevertheless, the algebra properties stated in Lemma 5.9 ensure that it is the case.

We look for a fixed point of the operator

$$
\mathcal{S}(h)=F+\widetilde{\mathcal{G}} \circ \mathcal{F}(h) .
$$

Next proposition gives its existence and uniqueness. From it, using that the function $Q$ of Theorem 5.5 is also a fixed point of $\mathcal{S}$ in the overlapping domain, one deduces Theorem 5.10.

Proposition 5.12. Consider $\rho_{0}$ and $\sigma_{0}$ the constants given by Theorem 5.5, $\rho_{1}>\rho_{0}, \kappa_{0}>0$ and $\delta_{0}>0$. Then, for $G_{0}$ big enough, there exists a constant $b_{1}>0$ independent of $G_{0}$ and $\mu$ such that the operator $\mathcal{S}$ has a unique fixed point $Q^{u} \in B\left(b_{1} \mu G_{0}^{-6}\right) \subset \widetilde{\mathcal{Y}}_{1, \rho_{1}, \kappa_{0}, \delta_{0}, \sigma_{0}}$.

Moreover, the (not necessarily convergent) Fourier series $L_{1}^{u}$ in (52) satisfies (64) and $Q^{u}$ satisfies [65].

To prove this proposition we first state the following technical lemma.

Lemma 5.13. The functions $Q_{0}$ and $\widehat{U}_{1}$, defined in (39) and (36) respectively, satisfy that $Q_{0} \in$ $\widetilde{\mathcal{Y}}_{1 / 2, \rho_{1}, \kappa_{0}, \delta_{0}, \sigma_{0}}$ and $\widehat{U}_{1} \circ A \in \mathcal{Y}_{2, \rho_{1}, \kappa_{0}, \delta_{0}, \sigma_{0}}$, where $A$ is the map in (38). Moreover,

$$
\begin{aligned}
\llbracket Q_{0} \rrbracket_{1 / 2, \sigma_{0}} & \leq K \mu G_{0}^{-4} \\
\left\|\widehat{U}_{1} \circ A\right\|_{2, \sigma_{0}} & \leq K \mu G_{0}^{-6} .
\end{aligned}
$$

Proof. For the first bound, we can write the function $Q_{0}$ in (39) as

$$
Q_{0}(v, \xi)=\widetilde{Q}_{0}(v, \xi)+\widetilde{\mathcal{G}}\left(\widehat{U}_{0} \circ A\right)(v, \xi)
$$

where

$$
\widetilde{Q}_{0}(v, \xi)=\sum_{\ell<0} e^{i \ell G_{0}^{3}\left(v-v_{1}\right)} Q_{0}^{[\ell]}\left(v_{1}\right) e^{i \ell \xi}+Q_{0}^{[0]}\left(-\rho_{1}\right)+\sum_{\ell>0} e^{i \ell G_{0}^{3}\left(v-\bar{v}_{1}\right)} Q_{0}^{[\ell]}\left(\bar{v}_{1}\right) e^{i \ell \xi} .
$$

We bound the two terms. For the first one, let us point out that $v_{1}, \bar{v}_{1},-\rho_{1} \in D_{\infty, \rho_{0}}^{u}$ and therefore we can bound the function $Q_{0}$ in these points thanks to Lemma 5.8. Then, we can deduce for the function $\widetilde{Q}_{0}$ the bound $\llbracket \widetilde{Q}_{0} \rrbracket_{1 / 2, \sigma_{0}} \leq K \mu G_{0}^{-4}$. For the second term, we use Corollary 5.3 and Lemma 5.4 to obtain $\left\|\widehat{U}_{0} \circ A\right\|_{3 / 2, \sigma_{0}} \leq K \mu G_{0}^{-4}$. Then, applying Lemma 5.11 we obtain that $\llbracket \widetilde{\mathcal{G}}\left(\widehat{U}_{0} \circ A\right) \rrbracket_{1 / 2, \sigma_{0}} \leq K \mu G_{0}^{-4}$.

For the second bound it is enough to apply Corollary 5.3 and Lemma [5.4

Proof of Proposition 5.12, First, we see that $\mathcal{S}$ is well defined from $\widetilde{\mathcal{Y}}_{1, \rho_{1}, \kappa_{0}, \delta_{0}, \sigma_{0}}$ to itself. Indeed if $h \in \widetilde{\mathcal{Y}}_{1, \rho_{1}, \kappa_{0}, \delta_{0}, \sigma_{0}}$, using Lemmas 5.9 and 5.13 and the properties of the separatrix parameterization given in (14), one can easily see that $\mathcal{F}(h) \in \mathcal{Y}_{2, \rho_{1}, \kappa_{0}, \delta_{0}, \sigma_{0}}$, where $\mathcal{F}$ is the operator defined in (42). Therefore using the last statement of Lemma 5.11, we obtain that $\widetilde{\mathcal{G}} \circ \mathcal{F}(h) \in \widetilde{\mathcal{Y}}_{1, \rho_{1}, \kappa_{0}, \delta_{0}, \sigma_{0}}$. Then, using also (67), we can deduce that $\mathcal{S}(h) \in \widetilde{\mathcal{Y}}_{1, \rho_{1}, \kappa_{0}, \delta_{0}, \sigma_{0}}$.

We bound the first iteration

$$
\mathcal{S}(0)(v, \xi)=F(v, \xi)+\widetilde{\mathcal{G}} \circ \mathcal{F}(0)(v, \xi) .
$$

Recall that $\mathcal{F}(0)$ has been defined in (56). Applying Lemmas 5.9 and 5.13 and Corollary 4.3 , it is easy to see that $\mathcal{F}(0) \in \mathcal{Y}_{2, \sigma_{0}}$ and $\|\mathcal{F}(0)\|_{2, \sigma_{0}} \leq K \mu G_{0}^{-6}$. Then, applying the last statement of Lemma 5.11] and taking into account (67), we obtain that there exists a constant $b_{1}>0$ such that

$$
\|\mathcal{S}(0)\|_{1, \sigma_{0}} \leq \frac{b_{1}}{2} \mu G_{0}^{-6}
$$


Now we prove that the operator $\mathcal{S}$ is contractive in $B\left(b_{1} \mu G_{0}^{-6}\right) \subset \widetilde{\mathcal{Y}}_{1, \rho_{1}, \kappa_{0}, \delta_{0}, \sigma_{0}}$. Take $h_{1}, h_{2} \in B\left(b_{1} \mu G_{0}^{-6}\right) \subset$ $\widetilde{\mathcal{Y}}_{1, \rho_{1}, \kappa_{0}, \delta_{0}, \sigma_{0}}$ and recall that

$$
\mathcal{S}\left(h_{2}\right)-\mathcal{S}\left(h_{1}\right)=\widetilde{\mathcal{G}}\left(\mathcal{F}\left(h_{2}\right)-\mathcal{F}\left(h_{1}\right)\right) .
$$

We start by bounding $\mathcal{F}\left(h_{2}\right)-\mathcal{F}\left(h_{1}\right)$. To this end, we use formula (57). Then, using Lemmas 5.9 and 5.13 the properties of the separatrix parameterization given in Corollary 4.3 and that $h_{1}, h_{2} \in B\left(b_{0} \mu G_{0}^{-6}\right) \subset$ $\widetilde{\mathcal{Y}}_{1, \rho_{1}, \kappa_{0}, \delta_{0}, \sigma_{0}}$, we obtain

$$
\left\|\mathcal{F}\left(h_{2}\right)-\mathcal{F}\left(h_{1}\right)\right\|_{2, \sigma_{0}} \leq K \mu G_{0}^{-5 / 2} \llbracket h_{2}-h_{1} \rrbracket_{1, \sigma_{0}} .
$$

Then, since $h_{1}, h_{2} \in \widetilde{\mathcal{Y}}_{1, \rho_{1}, \kappa_{0}, \delta_{0}, \sigma_{0}}$ and using Lemma 5.11, we can conclude that

$$
\llbracket \mathcal{S}\left(h_{2}\right)-\mathcal{S}\left(h_{1}\right) \rrbracket_{1, \sigma_{0}} \leq K\left\|\mathcal{F}\left(h_{2}\right)-\mathcal{F}\left(h_{1}\right)\right\|_{1, \sigma_{0}} \leq K \mu G_{0}^{-5 / 2} \llbracket h_{2}-h_{1} \rrbracket_{1, \sigma_{0}},
$$

and therefore, taking $G_{0}$ large enough, it is contractive and has a unique fixed point in $B\left(b_{1} \mu G_{0}^{-6}\right) \subset$ $\widetilde{\mathcal{Y}}_{1, \rho_{1}, \kappa_{0}, \delta_{0}, \sigma_{0}}$. This completes the first part of the proposition.

To prove the second part, we introduce

$$
F_{1}(v, \xi)=\sum_{\ell<0} e^{i \ell G_{0}^{3}\left(v-v_{1}\right)} L_{1}^{u[\ell]}\left(v_{1}\right) e^{i \ell \xi}+L_{1}^{u[0]}\left(-\rho_{1}\right)+\sum_{\ell>0} e^{i \ell G_{0}^{3}\left(v-\bar{v}_{1}\right)} L_{1}^{u[\ell]}\left(\bar{v}_{1}\right) e^{i \ell \xi},
$$

where $L_{1}^{u}$ was defined in (52). By (53), $F_{1} \in \widetilde{\mathcal{Y}}_{1, \rho_{1}, \kappa_{0}, \delta_{0}, \sigma_{0}}$ and

$$
\llbracket F_{1} \rrbracket_{1, \sigma_{0}} \leq K \mu G_{0}^{-6} .
$$

Then, since their Fourier coefficients coincide, we have that

$$
L_{1}^{u}=F_{1}+\widetilde{\mathcal{G}}\left(\widehat{U}_{1} \circ A\right) .
$$

Hence, inequality (64) follows from the estimate for $F_{1}$ just obtained, Lemma 5.13 and the second statement of Lemma 5.11.

Finally, to prove inequality (65) we note that, since $Q^{u}$ is a fixed point of $\mathcal{S}$,

$$
\left\|Q^{u}-L_{1}^{u}\right\|_{1, \sigma_{0}} \leq\left\|\mathcal{S}\left(Q^{u}\right)-\mathcal{S}(0)\right\|_{1, \sigma_{0}}+\left\|\mathcal{S}(0)-L_{1}^{u}\right\|_{1, \sigma_{0}} .
$$

The first term in the right hand side can be bounded using that $\mathcal{S}$ is Lipschitz,

$$
\begin{aligned}
\left\|\mathcal{S}\left(Q^{u}\right)-\mathcal{S}(0)\right\|_{1, \sigma_{0}} & \leq \llbracket \mathcal{S}\left(Q^{u}\right)-\mathcal{S}(0) \rrbracket_{1, \sigma_{0}} \\
& \leq K \mu G_{0}^{-5 / 2} \llbracket Q^{u} \rrbracket_{1, \sigma_{0}} \\
& \leq K b_{1} \mu^{2} G_{0}^{-17 / 2} .
\end{aligned}
$$

To obtain a bound of the second term in the right hand side we note that

$$
\mathcal{S}(0)-L_{1}^{u}=F-F_{1}+\widetilde{\mathcal{G}}\left(\mathcal{F}(0)-\widehat{U}_{1} \circ A\right) .
$$

Then, by (54),

and, by Lemma 5.13

$$
\left\|F-F_{1}\right\|_{1, \sigma_{0}} \leq K^{2} \mu^{2} G_{0}^{-8}
$$

$$
\left\|\mathcal{F}(0)-\widehat{U}_{1} \circ A\right\|_{2, \sigma_{0}}=\left\|\frac{1}{2 \widetilde{y}_{\mathrm{h}}^{2}}\left(\partial_{v} Q_{0}-\frac{1}{\widehat{r}_{\mathrm{h}}^{2}} \partial_{\xi} Q_{0}\right)^{2}+\frac{1}{2 \widetilde{r}_{\mathrm{h}}^{2}} \partial_{\xi} Q_{0}^{2}\right\|_{2, \sigma_{0}} \leq K^{2} \mu^{2} G_{0}^{-8} .
$$

The claim follows then applying the second statement of Lemma $\left[5.11\right.$ to $\widetilde{\mathcal{G}}\left(\mathcal{F}(0)-\widehat{U}_{1} \circ A\right)$. 


\subsection{Extension of the parameterization of the unstable manifold by the flow}

From Theorem 5.10 we have a formal parameterization of the unstable invariant manifold as not necessarily convergent Fourier series in the domain $D_{\rho_{1}, \kappa_{0}, \delta_{0}}^{u, s}$. This parameterization is given by

$$
\widetilde{\Gamma}^{u}(v, \xi)=\left(\begin{array}{c}
\widetilde{r} \\
\widetilde{y} \\
\widetilde{\phi} \\
\widetilde{G}
\end{array}\right)=\left(\begin{array}{c}
\widetilde{r}_{\mathrm{h}}(v) \\
\widetilde{y}_{\mathrm{h}}(v)^{-1}\left(\partial_{v} T^{u}(v, \xi)-\widetilde{r}_{\mathrm{h}}(v)^{-2} \partial_{\xi} T^{u}(v, \xi)\right) \\
\xi+\widetilde{\alpha}_{\mathrm{h}}(v) \\
\partial_{\xi} T^{u}(v, \xi)
\end{array}\right),
$$

where

$$
T^{u}=T_{0}+Q_{0}^{u}+Q^{u}
$$

with

$$
T_{0}(v, \xi)=S_{0}\left(\widetilde{r}_{\mathrm{h}}(v), \xi+\widetilde{\alpha}(v)\right),
$$

$S_{0}$ was introduced in (21), $Q_{0}^{u}$ in (39) and $Q^{u}$ is given by Theorem 5.10. Analogously for $\widetilde{\Gamma}^{s}$.

To compute its difference is necessary to have the parameterizations of both manifolds defined in a common (real) domain. However, since $\widetilde{y}_{\mathrm{h}}(0)=0$, it is no possible to extend these parameterizations to a common domain containing a real interval. To overcome this difficulty we extend the unstable manifold using a different parameterization as has been explained in Section 5.1

\subsubsection{From Hamilton-Jacobi parameterizations to parameterizations invariant by the flow}

The first step is to look for a change of variables of the form

$$
\mathrm{Id}+g:(v, \xi) \mapsto\left(v+g_{1}(v, \xi), \xi+g_{2}(v, \xi)\right), g=\left(g_{1}, g_{2}\right),
$$

in such a way that, applied to (68), $\widehat{\Gamma}^{u}=\widetilde{\Gamma}^{u} \circ(\operatorname{Id}+g)$ satisfies

$$
\Phi_{t}\left(\widehat{\Gamma}^{u}(v, \xi)\right)=\widehat{\Gamma}^{u}\left(v+t, \xi-G_{0}^{3} t\right),
$$

where $\Phi_{t}$ is the flow associated to the Hamiltonian (17). Denoting by $X$ the vector field generated by this Hamiltonian, this equation is equivalent to

$$
\mathcal{L}\left(\widehat{\Gamma}^{u}\right)=X \circ \widehat{\Gamma}^{u}
$$

where the operator $\mathcal{L}$ defined in (34) is understood to be acting on each component of $\widehat{\Gamma}^{u}$. In fact, writing $X=X_{0}+X_{1}$, being $X_{0}$ the vector field for $\mu=0$, and denoting by $\Gamma_{0}$ the parameterization defined by equations (68) replacing $T^{u}$ by $T_{0}$, it is an immediate computation to see that

$$
\mathcal{L}\left(\Gamma_{0}\right)=X_{0} \circ \Gamma_{0}
$$

Hence, the condition one needs to impose on $g$ to ensure that $\widehat{\Gamma}^{u}=\widetilde{\Gamma}^{u} \circ(\operatorname{Id}+g)$ satisfies (70) is

$$
\partial_{v} \widetilde{\Gamma}^{u} \circ(\operatorname{Id}+g)\left(1+\mathcal{L}\left(g_{1}\right)\right)+\partial_{\xi} \widetilde{\Gamma}^{u} \circ(\operatorname{Id}+g)\left(-G_{0}^{3}+\mathcal{L}\left(g_{2}\right)\right)=X \circ \widetilde{\Gamma}^{u} \circ(\operatorname{Id}+g) .
$$

We remark that, unlike the Hamilton-Jacobi equation, this is a system of four partial differential equations. However, the symplectic structure implies that it is only necessary to solve two of them, which we choose to be the first and the third ones. Then, the other two are also fulfilled.

By the definition of $T_{0}$ in (69), (68) and using (71), equation (72) is equivalent to

$$
\mathcal{L}(g)=\widetilde{F} \circ(\mathrm{Id}+g), \quad \widetilde{F}=\left(\begin{array}{l}
A^{u} \\
B^{u}
\end{array}\right)
$$


where

$$
\begin{aligned}
A^{u} & =\frac{1}{\widetilde{y}_{\mathrm{h}}^{2}}\left(\partial_{v} T_{1}^{u}-\frac{1}{\widetilde{\mathrm{r}}_{\mathrm{h}}^{2}} \partial_{\xi} T_{1}^{u}\right) \\
B^{u} & =\left[\frac{1}{\widetilde{\widetilde{r}}_{\mathrm{h}}^{2}} \partial_{\xi} T_{1}^{u}-\frac{1}{\widetilde{r}_{\mathrm{h}}^{2} \widetilde{y}_{\mathrm{h}}^{2}}\left(\partial_{v} T_{1}^{u}-\frac{1}{\widetilde{r}_{\mathrm{h}}^{2}} \partial_{\xi} T_{1}^{u}\right)\right]
\end{aligned}
$$

and

$$
T_{1}^{u}=Q_{0}^{u}+Q^{u} .
$$

We emphasize that equations (172) or their equivalent (73) have to be understood at the level of formal Fourier series, since $T^{u}$ and, consequently, $\widetilde{\Gamma}^{u}$, are this kind of objects. In particular, the change Id $+g$ will be a formal Fourier series. Hence, it is necessary to give a meaningful definition of the composition of formal Fourier series. This will be accomplished taking into account that the formal change of variables Id $+g$ is close to the identity and using Taylor's formula.

In order to find the change Id $+g$, we define for $g=\left(g_{1}, g_{2}\right) \in \mathcal{Y}_{\nu, \rho, \kappa, \delta, \sigma} \times \mathcal{Y}_{\nu, \rho, \kappa, \delta, \sigma}$, where $\mathcal{Y}_{\nu, \rho, \kappa, \delta, \sigma}$ is the Banach space defined in (62), the norm

$$
\|g\|_{\nu, \sigma}=\left\|g_{1}\right\|_{\nu, \sigma}+G_{0}^{-3}\left\|g_{2}\right\|_{\nu, \sigma} .
$$

The following two technical lemmas summarize the properties of the composition of formal Fourier series we need. The second is an immediate consequence of the first.

Lemma 5.14. Fix constants $\sigma^{\prime}<\sigma, \delta^{\prime}<\delta, \rho^{\prime}<\rho$ and $\kappa^{\prime}>\kappa$ such that $\left(\log \kappa^{\prime}-\log \kappa\right) / 2<\sigma-\sigma^{\prime}$ and take $h \in \mathcal{Y}_{\nu, \rho, \kappa, \delta, \sigma}$. Its derivatives, as defined in (63), satisfy $\partial_{v}^{m} \partial_{\xi}^{n} h \in \mathcal{Y}_{\nu, \rho^{\prime}, \kappa^{\prime}, \delta^{\prime}, \sigma^{\prime}}$ and

$$
\left\|\partial_{v}^{m} \partial_{\xi}^{n} h\right\|_{\nu, \sigma^{\prime}} \leq\left(\frac{\kappa^{\prime}}{\kappa}\right)^{\nu} \frac{G_{0}^{3 m} m ! n !}{\left(\kappa^{\prime}-\kappa\right)^{m}\left(\sigma-\sigma^{\prime}-\left(\log \kappa^{\prime}-\log \kappa\right) / 2\right)^{n}}\|h\|_{\nu, \sigma} .
$$

Proof. Fix constants $\sigma^{\prime}<\sigma, \delta^{\prime}<\delta, \rho^{\prime}<\rho$ and $\kappa^{\prime}>\kappa$ such that $\left(\log \kappa^{\prime}-\log \kappa\right) / 2<\sigma-\sigma^{\prime}$ and take $h \in \mathcal{Y}_{\nu, \rho, \kappa, \delta, \sigma}$. By (63),

$$
\left(\partial_{v}^{m} \partial_{\xi}^{n} h\right)^{[\ell]}=(i \ell)^{n} \partial_{v}^{m} h^{[\ell]},
$$

and, by Cauchy's formula,

$$
\partial_{v}^{m} h^{[\ell]}(v)=\frac{m !}{2 \pi i} \int_{\gamma_{v}} \frac{h^{[\ell]}(z)}{(z-v)^{m+1}} d z,
$$

where $\gamma_{v}$ is a curve with index 1 with respect to $v$ in $D_{\rho, \kappa, \delta}^{u}$. In particular, since we will measure the norm of this function in $D_{\rho, \kappa, \delta}^{u}$ and for $G_{0}$ big enough, one can choose that $\delta-\delta^{\prime}>\left(\kappa^{\prime}-\kappa\right) G_{0}^{-3}$ and $\rho-\rho^{\prime}>\left(\kappa^{\prime}-\kappa\right) G_{0}^{-3}$, and we can take $\gamma_{v}:[0,2 \pi] \rightarrow \mathbb{C}: t \mapsto v+\left(\kappa^{\prime}-\kappa\right) G_{0}^{-3} e^{i t}$. Hence, by (59)

$$
\begin{aligned}
\left\|\left(\partial_{v}^{m} \partial_{\xi}^{n} h\right)^{[\ell]}\right\|_{\nu+\ell / 2, \nu-\ell / 2} & =\sup _{v \in D_{\rho^{\prime}, \kappa^{\prime}, \delta^{\prime}}^{u}}\left|(v-i / 3)^{\nu-\ell / 2}(v+i / 3)^{\nu+\ell / 2}\left(\partial_{v}^{m} \partial_{\xi}^{n} h\right)^{[\ell]}(v)\right| \\
& \leq \frac{\ell^{n} m !}{\left(\kappa^{\prime}-\kappa\right)^{m} G_{0}^{-3 m}}\left(\frac{\kappa^{\prime}}{\kappa}\right)^{\nu+|\ell| / 2}\left\|h^{[\ell]}\right\|_{\nu+\ell / 2, \nu-\ell / 2},
\end{aligned}
$$

where we have used that

$$
\sup _{v \in D_{\rho^{\prime}, \kappa^{\prime}, \delta^{\prime}}^{u}}\left|\frac{v \pm i / 3}{v+\left(\kappa^{\prime}-\kappa\right) G_{0}^{-3} e^{i t} \pm i / 3}\right| \leq \frac{\kappa^{\prime}}{\kappa} .
$$

Then, by [61), since $h \in \mathcal{Y}_{\nu, \rho, \kappa, \delta, \sigma}$,

$$
\begin{aligned}
\left\|\partial_{v}^{m} \partial_{\xi}^{n} h\right\|_{\nu, \sigma^{\prime}} & \leq \sum_{\ell \in \mathbb{Z}}\left\|\left(\partial_{v}^{m} \partial_{\xi}^{n} h\right)^{[\ell]}\right\|_{\nu+\ell / 2, \nu-\ell / 2} e^{|\ell| \sigma^{\prime}} \\
& \leq \frac{m !}{\left(\kappa^{\prime}-\kappa\right)^{m} G_{0}^{-3 m}}\left(\frac{\kappa^{\prime}}{\kappa}\right)^{\nu}\|h\|_{\nu, \sigma} \sum_{\ell \in \mathbb{Z}} \ell^{n}\left(\frac{\kappa^{\prime}}{\kappa}\right)^{|\ell| / 2} e^{|\ell|\left(\sigma^{\prime}-\sigma\right)} \\
& \leq 2 \frac{m ! n ! G_{0}^{3 m}}{\left(\kappa^{\prime}-\kappa\right)^{m}\left(\sigma-\sigma^{\prime}-\left(\log \kappa^{\prime}-\log \kappa\right) / 2\right)^{n}}\left(\frac{\kappa^{\prime}}{\kappa}\right)^{\nu}\|h\|_{\nu, \sigma},
\end{aligned}
$$


where we have used that, for $b>0, \sum_{\ell \geq 0} \ell^{n} e^{-b \ell} \leq 2 n ! / b^{n}$.

Lemma 5.15. Given $g=\left(g_{1}, g_{2}\right)$, let $\mathrm{Id}+g:(v, \xi) \mapsto\left(v+g_{1}(v, \xi), \xi+g_{2}(v, \xi)\right)$. We define the formal composition of formal Fourier series

$$
\begin{aligned}
h \circ(\operatorname{Id}+g)(v, \xi) & =h\left(v+g_{1}(v, \xi), \xi+g_{2}(v, \xi)\right) \\
& =\sum_{m=0}^{\infty} \frac{1}{m !} \sum_{n=0}^{m}\left(\begin{array}{c}
m \\
n
\end{array}\right) \partial_{v}^{m-n} \partial_{\xi}^{n} h(v, \xi) g_{1}^{m-n}(v, \xi) g_{2}^{n}(v, \xi) .
\end{aligned}
$$

Fix constants $\sigma^{\prime}<\sigma, \rho^{\prime}<\rho, \delta^{\prime}<\delta$ and $\kappa^{\prime}>\kappa$ such that $\left(\log \kappa^{\prime}-\log \kappa\right) / 2<\sigma-\sigma^{\prime}$. Let $\theta=$ $\min \left\{\kappa^{\prime}-\kappa, \sigma-\sigma^{\prime}-\left(\log \kappa^{\prime}-\log \kappa\right) / 2\right\}>2 \eta>0$. Then,

- If $h \in \mathcal{Y}_{\nu, \rho, \kappa, \delta, \sigma}, g=\left(g_{1}, g_{2}\right) \in \mathcal{Y}_{0, \rho^{\prime}, \kappa^{\prime}, \delta^{\prime}, \sigma^{\prime}} \times \mathcal{Y}_{0, \rho^{\prime}, \kappa^{\prime}, \delta^{\prime}, \sigma^{\prime}}$ and $\|g\|_{0, \sigma^{\prime}} \leq \eta G_{0}^{-3}$, we have that $\widetilde{h}=h \circ(\mathrm{Id}+g)$ satisfies $\widetilde{h} \in \mathcal{Y}_{\nu, \rho^{\prime}, \kappa^{\prime}, \delta^{\prime}, \sigma^{\prime}}$ and

$$
\|\widetilde{h}\|_{\nu, \sigma^{\prime}} \leq\left(\frac{\kappa^{\prime}}{\kappa}\right)^{\nu}\left(1-\frac{2 \eta}{\theta}\right)^{-1}\|h\|_{\nu, \sigma} .
$$

- Moreover, if $\|g\|_{0, \sigma^{\prime}},\|\widehat{g}\|_{0, \sigma^{\prime}} \leq \eta G_{0}^{-3}$, then $f=h \circ(\mathrm{Id}+g)-h \circ(\mathrm{Id}+\widehat{g})$ satisfies

$$
\|f\|_{\nu, \sigma^{\prime}} \leq \frac{2 G_{0}^{3}}{\delta}\left(\frac{\kappa^{\prime}}{\kappa}\right)^{\nu}\left(1-\frac{2 \eta}{\theta}\right)^{-2}\|h\|_{\nu, \sigma}\|g-\widehat{g}\|_{0, \sigma^{\prime}} .
$$

Theorem 5.16. Let $\rho_{1}, \delta_{0}$ and $\kappa_{0}$ and $\sigma_{0}$ be the constants given by Theorem 5.10. Let $\sigma_{1}<\sigma_{0}, \rho_{2}<\rho_{1}$, $\delta_{1}<\delta_{0}$ and $\kappa_{1}>\kappa_{0}$ such that $\left(\log \kappa_{1}-\log \kappa_{0}\right) / 2<\sigma_{0}-\sigma_{1}$ be fixed. Then, for $G_{0}$ big enough, there exists a (not necessarily convergent) Fourier series $g=\left(g_{1}, g_{2}\right) \in \mathcal{Y}_{0, \rho_{2}, \kappa_{1}, \delta_{1}, \sigma_{1}} \times \mathcal{Y}_{0, \rho_{2}, \kappa_{1}, \delta_{1}, \sigma_{1}}$ satisfying

$$
\|g\|_{0, \sigma_{1}} \leq b_{2} \mu G_{0}^{-4}
$$

where $b_{2}>0$ is a constant independent of $\mu$ and $G_{0}$, such that

$$
\widehat{\Gamma}^{u}=\widetilde{\Gamma}^{u} \circ(\mathrm{Id}+g),
$$

satisfies (70).

Proof. The function $g$ is found as a solution of equation (73). The following auxiliary lemma provides the appropriate bounds on the function $\widetilde{F}$ introduced in (73).

Lemma 5.17. $\widetilde{F} \in \mathcal{Y}_{1 / 2, \rho_{1}, \kappa_{0}, \delta_{0}, \sigma_{0}} \times \mathcal{Y}_{1 / 2, \rho_{1}, \kappa_{0}, \delta_{0}, \sigma_{0}}$ and $\|\widetilde{F}\|_{1 / 2, \sigma_{0}} \leq K \mu G_{0}^{-4}$.

Proof of Lemma 5.17. It follows directly from the bound on $Q_{0}^{u}$ in Lemma 5.13 and the bound on $Q^{u}$ in Theorem 5.10 .

Using the operator $\widetilde{\mathcal{G}}$ in (66), we rewrite equation (73) as

$$
g=\widetilde{\mathcal{G}} \circ \widetilde{F} \circ(\mathrm{Id}+g)
$$

and we look for $g$ as a fixed point of this operator.

Taking $G_{0}$ large enough, by Lemmas 5.17 and 5.15 the map $g \mapsto \widetilde{F} \circ(\mathrm{Id}+g)$ is well defined and sends the ball $B\left(K \mu G_{0}^{-4}\right) \subset \mathcal{Y}_{0, \rho_{2}, \kappa_{1}, \delta_{1}, \sigma_{1}} \times \mathcal{Y}_{0, \rho_{2}, \kappa_{1}, \delta_{1}, \sigma_{1}}$ to $\mathcal{Y}_{0, \rho_{2}, \kappa_{1}, \delta_{1}, \sigma_{1}} \times \mathcal{Y}_{0, \rho_{2}, \kappa_{1}, \delta_{1}, \sigma_{1}}$. Also, by Lemma 5.17

$$
\left\|\widetilde{F} \circ(\mathrm{Id}+g)_{\mid g=0}\right\|_{1 / 2, \sigma_{1}} \leq\|\widetilde{F}\|_{1 / 2, \sigma_{0}} \leq K \mu G_{0}^{-4}
$$

which implies, by Lemma 5.11, that

$$
\|\widetilde{\mathcal{G}} \circ \widetilde{F}\|_{0, \sigma_{1}} \leq K \mu G_{0}^{-4}
$$


It only remains to see that the map defined by (74) is Lipschitz. But this is straightforward, since, by Lemma 5.15 for any $g, \widehat{g} \in B\left(K \mu G_{0}^{-4}\right) \subset \mathcal{Y}_{0, \rho_{2}, \kappa_{1}, \delta_{1}, \sigma_{1}} \times \mathcal{Y}_{0, \rho_{2}, \kappa_{1}, \delta_{1}, \sigma_{1}}$,

$$
\|\widetilde{F} \circ(\mathrm{Id}+g)-\widetilde{F} \circ(\mathrm{Id}+\widehat{g})\|_{1 / 2, \sigma_{1}} \leq \widetilde{K} \mu G_{0}^{-1}\|g-\widehat{g}\|_{0, \sigma_{1}},
$$

where the constant $\widetilde{K}$ depends on the reduction of the domain. Finally, using the last inequality and Lemma 5.11, we have that

$$
\|\widetilde{\mathcal{G}}(\widetilde{F} \circ(\operatorname{Id}+g)-\widetilde{F} \circ(\operatorname{Id}+\widehat{g}))\|_{0, \sigma_{1}} \leq K \widetilde{K} \mu G_{0}^{-1}\|g-\widehat{g}\|_{0, \sigma_{1}} .
$$

\subsubsection{Analytic extension of the unstable manifold by the flow parameterization}

Now we can extend the flow parameterization of the unstable manifold given by Theorem 5.16, whose Fourier coefficients are defined, up to now, for $v \in D_{\rho_{2}, \kappa_{1}, \delta_{1}}^{u}$, to $D_{\kappa_{1}, \delta_{1}}^{\text {flow }}$ defined in (50) (see Figure 8). This extension is obtained using the flow of the Hamiltonian (17).

We look for a parameterization of the unstable manifold $\widehat{\Gamma}^{u}$ satisfying (70), defined in $D_{\kappa_{1}, \delta_{1}}^{\text {flow }}$ and which coincides with the one given by Theorem 5.16 in $D_{\rho_{2}, \kappa_{1}, \delta_{1}}^{u} \cap D_{\kappa_{1}, \delta_{1}}^{\mathrm{flow}}$.

Using the notations of the previous section, where $X \stackrel{\rho_{2}, \kappa_{1}, \delta_{1}}{=} X_{0}+X_{1}$ is the vector field generated by the Hamiltonian (17), and introducing $\Gamma_{1}^{u}$ such that $\widehat{\Gamma}^{u}=\Gamma_{0}+\Gamma_{1}^{u}$, where $\Gamma_{0}$ is obtained from (68) with $T_{0}$ instead of $T^{u}$, and satisfies $\mathcal{L}\left(\Gamma_{0}\right)=X_{0} \circ \Gamma_{0}$, equation (70) becomes

$$
\widetilde{\mathcal{L}}\left(\Gamma_{1}^{u}\right)=\widetilde{\mathcal{F}}\left(\Gamma_{1}^{u}\right),
$$

where

$$
\widetilde{\mathcal{L}}(\Gamma)=\mathcal{L}(\Gamma)-D X_{0}\left(\Gamma_{0}\right) \Gamma
$$

and

$$
\widetilde{\mathcal{F}}(\Gamma)=X_{0}\left(\Gamma_{0}+\Gamma\right)-X_{0}\left(\Gamma_{0}\right)-D X_{0}\left(\Gamma_{0}\right) \Gamma+X_{1}\left(\Gamma_{0}+\Gamma\right) .
$$

We will find a solution of this equation whose initial condition is given the formal Fourier series obtained by Theorem [5.16. For this reason, we introduce the space of formal Fourier series

$$
\mathcal{Z}_{\nu, \kappa, \delta, \sigma}=\left\{h(v, \xi)=\sum_{\ell \in \mathbb{Z}} h^{[\ell]}(v) e^{i \ell \xi}: h^{[\ell]} \in\left(\widetilde{\mathcal{X}}_{\nu+\ell / 2, \nu-\ell / 2, \kappa, \delta}\right)^{4},\|h\|_{\nu, \sigma}<\infty\right\},
$$

where

$$
\widetilde{\mathcal{X}}_{\nu_{-}, \nu_{+}, \kappa, \delta}=\left\{h: D_{\kappa, \delta}^{\text {flow }} \rightarrow \mathbb{C}:\|h\|_{\nu_{-}, \nu_{+}}<\infty\right\},
$$

the norm $\|\cdot\|_{\nu_{-}, \nu_{+}}$is the one defined in (59), but taking the supremum in $D_{\kappa, \delta}^{\text {flow }}$ and,

$$
\|h\|_{\nu, \sigma}=\sum_{i=1}^{4}\left\|h_{i}\right\|_{\nu, \sigma}
$$

where the norm $\|\cdot\|_{\nu, \sigma}$ in the right hand side above is the one in (62). We remark that, since $\pm i / 3$ are at a distance $\mathcal{O}(1)$ of the domain $D_{\kappa, \delta}^{\text {flow }}$, the norms $\|\cdot\|_{\nu, \sigma}$ are equivalent for all $\nu$. Therefore, we only work with $\nu=0$.

Let $\Psi(v)$ be any fundamental matrix of the linear system

$$
\dot{z}(v)=D X_{0}\left(\Gamma_{0}(v, \xi)\right) z(v), \quad v \in D_{\kappa_{1}, \delta_{1}}^{\text {flow }} .
$$

In fact, the system above does not depend on $\xi$. As a consequence, the matrix $\Psi$ satisfies

$$
\mathcal{L} \Psi=D X_{0}\left(\Gamma_{0}\right) \Psi .
$$


Since $\Gamma_{0}(v, \xi)$ is well defined and bounded for $v \in D_{\kappa_{1}, \delta_{1}}^{\text {flow }}$ and $D_{\kappa_{1}, \delta_{1}}^{\text {flow }}$ is bounded, we have that there exists $K>0$ such that

$$
\sup _{v \in D_{\kappa_{1}, \delta_{1}}^{\text {flow }}} \max \left\{\|\Psi(v)\|_{0, \sigma},\left\|\Psi(v)^{-1}\right\|_{0, \sigma}\right\} \leq K,
$$

in any matrix norm.

We choose $v_{2}$ at the top of the domain $D_{\kappa_{1}, \delta_{1}}^{\text {flow }}$ and define $\widetilde{\mathcal{G}}$ like in (66). Lemma 5.11 applies without further modification. Then, it is a easy computation to check that a left inverse of the operator $\widetilde{\mathcal{L}}$ in (76) is

$$
\widehat{\mathcal{G}}(\Gamma)=\Psi \widetilde{\mathcal{G}}\left(\Psi^{-1} \Gamma\right) .
$$

Since we want to obtain an analytic continuation of the parameterization of the invariant manifold given by Theorem 5.16. we introduce an initial condition defining

$$
\begin{aligned}
\Gamma_{1}^{0}(v, \xi)= & \sum_{\ell<0} \Psi(v) \Psi^{-1}\left(v_{2}\right) \Gamma_{1}^{[\ell]}\left(v_{2}\right) e^{i \ell G_{0}^{3}\left(v-v_{2}\right)} e^{i \ell \xi} \\
& +\sum_{\ell>0} \Psi(v) \Psi^{-1}\left(\bar{v}_{2}\right) \Gamma_{1}^{[\ell]}\left(\bar{v}_{2}\right) e^{i \ell G_{0}^{3}\left(v-\bar{v}_{2}\right)} e^{i \ell \xi} \\
& +\Psi(v) \Psi^{-1}\left(-\rho_{2}\right) \Gamma_{1}^{[0]}\left(-\rho_{2}\right),
\end{aligned}
$$

where $\Gamma_{1}^{[\ell]}$ are the Fourier coefficients of $\Gamma_{1}^{u}$ and therefore are already known at the points $v_{2}, \bar{v}_{2}$ and $-\rho_{2}$. The following lemma collects the properties we need about $\Gamma_{1}^{0}$.

Lemma 5.18. The function $\Gamma_{1}^{0}$ satisfies

- $\tilde{\mathcal{L}}\left(\Gamma_{1}^{0}\right)=0$ and

- $\Gamma_{1}^{0} \in \mathcal{Z}_{0, \kappa_{1}, \delta_{1}, \sigma_{1}}$ with $\left\|\Gamma_{1}^{0}\right\|_{0, \sigma_{1}}<K \mu G_{0}^{-4}$.

Proof. The first statement is straightforward. For the second one, it is enough to write

$$
\begin{aligned}
\Gamma_{1}^{u} & =\widehat{\Gamma}^{u}-\Gamma_{0} \\
& =\left(\widetilde{\Gamma}^{u} \circ(\operatorname{Id}+g)-\Gamma_{0} \circ(\operatorname{Id}+g)\right)+\left(\Gamma_{0} \circ(\operatorname{Id}+g)-\Gamma_{0}\right) .
\end{aligned}
$$

Now, using the formula of $\widetilde{\Gamma}^{u}$ in (68), the estimates given in Theorem 5.10, the estimates for $Q_{0}^{u}$ in Lemma 5.13 and the ones for $g$ given in Theorem 5.16. Lemma 5.15 for the composition and the fact that the domain $D_{\kappa_{1}, \delta_{1}}^{\text {flow }}$ only contains points at a distance of order one of the singularities $v= \pm i / 3$, one obtains the estimate of the second statement.

We rewrite equation (75) using the function $\Gamma_{1}^{0}$ and the operator $\widehat{\mathcal{G}}$ in (80) as

$$
\Gamma_{1}^{u}=\Gamma_{1}^{0}+\widehat{\mathcal{G}} \circ \widetilde{\mathcal{F}}\left(\Gamma_{1}^{u}\right)
$$

It is important to remark that the definition of $\widetilde{\mathcal{F}}$ in (777) involves the compositions $X_{0}\left(\Gamma_{0}+\Gamma_{1}^{u}\right)$ and $X_{1}\left(\Gamma_{0}+\Gamma_{1}^{u}\right)$ of formal Fourier series. Like in the previous section, in Lemma 5.15, this composition is defined through a Taylor series of the form

$$
X\left(\Gamma_{0}+\Gamma\right)=\sum_{k \geq 0} \frac{1}{k !} D^{k} X\left(\Gamma_{0}\right) \Gamma^{\otimes k} .
$$

However, this case is much simpler, since the vector fields $X_{0}$ and $X_{1}$ and the function $\Gamma_{0}$ are true functions, analytic and appropriately bounded in the domain $D_{\kappa_{1}, \delta_{1}}^{\text {flow }}$.

Lemma 5.19. Let $K>0$. Assume $\Gamma, \widetilde{\Gamma} \in \mathcal{Z}_{0, \kappa_{1}, \delta_{1}, \sigma_{1}}$ with $\|\Gamma\|_{0, \sigma_{1}},\|\widetilde{\Gamma}\|_{0, \sigma_{1}} \leq K \mu G_{0}^{-4}$. Then there exists $K^{\prime}>0$ such that, if $G_{0}$ is large enough, 
- defining $Y(\Gamma)=X_{0}\left(\Gamma_{0}+\Gamma\right)-X_{0}\left(\Gamma_{0}\right)-D X_{0}\left(\Gamma_{0}\right) \Gamma$, we have that $Y(\Gamma) \in \mathcal{Z}_{0, \kappa_{1}, \delta_{1}, \sigma_{1}}$ with

$$
\|Y(\Gamma)\|_{0, \sigma_{1}} \leq K^{\prime} \mu G_{0}^{-4},
$$

- $X_{1}\left(\Gamma_{0}+\Gamma\right) \in \mathcal{Z}_{0, \kappa_{1}, \delta_{1}, \sigma_{1}}$ with $\left\|X_{1}\left(\Gamma_{0}+\Gamma\right)\right\|_{0, \sigma_{1}} \leq K^{\prime} \mu G_{0}^{-4}$,

- $\|Y(\Gamma)-Y(\widetilde{\Gamma})\|_{0, \sigma_{1}} \leq K^{\prime} \mu G_{0}^{-4}\|\Gamma-\widetilde{\Gamma}\|_{0, \sigma_{1}}$,

- $\left\|X_{1}\left(\Gamma_{0}+\Gamma\right)-X_{1}\left(\Gamma_{0}+\widetilde{\Gamma}\right)\right\|_{0, \sigma_{1}} \leq K^{\prime} \mu G_{0}^{-4}\|\Gamma-\widetilde{\Gamma}\|_{0, \sigma_{1}}$.

Proof. Cauchy estimates imply that

$$
\left\|D^{k} X_{0}\left(\Gamma_{0}\right)\right\|_{0, \sigma} \leq \widetilde{K} k !, \quad\left\|D^{k} X_{1}\left(\Gamma_{0}\right)\right\|_{0, \sigma} \leq \widetilde{K} \mu G_{0}^{-4} k ! .
$$

The claims follow then from formula (82).

Now we can claim

Proposition 5.20. Let $\kappa_{1}, \delta_{1}$ and $\sigma_{1}$ be the constants considered in Theorem 5.16. Then, there exists $b_{3}>0$ such that if $G_{0}$ is large enough, the fixed point equation (81) has a unique solution $\Gamma_{1}^{u} \in B\left(b_{3} \mu G_{0}^{-4}\right) \subset \mathcal{Z}_{0, \kappa_{1}, \delta_{1}, \sigma_{1}}$.

Proof. By Lemmas 5.185 .19 and the algebra properties of $\mathcal{Z}_{0, \kappa_{1}, \delta_{1}, \sigma_{1}}$, the map $\widetilde{\mathcal{K}}: \Gamma \mapsto \Gamma_{1}^{0}+\widetilde{G} \circ \widetilde{\mathcal{F}}(\Gamma)$ is well defined from $B\left(K \mu G_{0}^{-4}\right) \subset \mathcal{Z}_{0, \kappa_{1}, \delta_{1}, \sigma_{1}}$ to $\mathcal{Z}_{0, \kappa_{1}, \delta_{1}, \sigma_{1}}$ for any $K>0$. Also, by Lemmas 5.18, 5.19 and 5.11 and using the bound (79) on the fundamental matrix $\Psi$, we have that

$$
\|\widetilde{\mathcal{K}}(0)\|_{0, \sigma_{1}}=\left\|\Gamma_{1}^{0}+\widehat{\mathcal{G}}\left(X_{1} \circ \Gamma_{0}\right)\right\|_{0, \sigma_{1}} \leq \frac{b_{3}}{2} \mu G_{0}^{-4} .
$$

for certain $b_{3}$ independent of $G_{0}$ and $\mu$.

Finally, Lemmas 5.19 and 5.11 imply that $\widetilde{\mathcal{K}}$ is Lipschitz with Lipschitz constant $K \mu G_{0}^{-4}$.

\subsubsection{From flow parameterization to Hamilton-Jacobi parameterization}

Finally we apply a change of variables to the parameterization $\widehat{\Gamma}^{u}=\Gamma_{0}+\Gamma_{1}^{u}$ obtained in Proposition 5.20 , which is invariant by the flow, in order to obtain an extension of the parameterization of the form (68) to the domain $\widetilde{D}_{\kappa_{2}, \delta_{2}}$ defined in (47) for some $\kappa_{2}>\kappa_{1}$ and $\delta_{2}>\delta_{1}$ (see Figure 7). Equivalently, we obtain an extension of the Fourier coefficients of $T^{u}$, solution of the Hamilton-Jacobi equation, to this domain. Like in the previous section, since this change of variables has to be found in a domain which is far from the singularities $v= \pm i / 3$, the procedure is rather simple.

We will find a change of variables of the form Id $+f$, with $f=\left(f_{1}, f_{2}\right)$, such that

$$
\begin{aligned}
& \pi_{1} \circ \widehat{\Gamma}^{u} \circ(\operatorname{Id}+f)(v, \xi)=\widetilde{r}_{\mathrm{h}}(v), \\
& \pi_{3} \circ \widehat{\Gamma}^{u} \circ(\operatorname{Id}+f)(v, \xi)=\widetilde{\alpha}_{\mathrm{h}}(v)+\xi,
\end{aligned}
$$

where $\pi_{i}$ denotes the projection on the $i$-th component. We will see that this change of variables is unique under certain conditions. Therefore, in $D_{\rho_{2}, \kappa_{1}, \delta_{1}}^{u} \cap \widetilde{D}_{\kappa_{2}, \delta_{2}}$ the change $\mathrm{Id}+f$ is the formal inverse of the change Id $+g$ obtained in Theorem 5.16 Then,

$$
\begin{aligned}
& \pi_{2} \circ \widehat{\Gamma}^{u} \circ(\operatorname{Id}+f)(v, \xi)=\widetilde{y}_{\mathrm{h}}(v)^{-1}\left(\partial_{v} T^{u}(v, \xi)-\widetilde{r}_{\mathrm{h}}(v)^{-2} \partial_{\xi} T^{u}(v, \xi)\right) \\
& \pi_{4} \circ \widehat{\Gamma}^{u} \circ(\operatorname{Id}+f)(v, \xi)=\partial_{\xi} T^{u}(v, \xi) .
\end{aligned}
$$

These equalities provide an analytic extension of the Fourier coefficients of $T^{u}$ given by Theorem 5.10

Taking into account that $\pi_{1} \circ \Gamma_{0}(v, \xi)=\widetilde{r}_{\mathrm{h}}(v)$ and $\pi_{3} \circ \Gamma_{0}(v, \xi)=\widetilde{\alpha}_{\mathrm{h}}(v)+\xi$ and that $\widehat{\Gamma}^{u}=\Gamma_{0}+\Gamma_{1}^{u}$, equations (83) are equivalent to

$$
f=\mathcal{P}(f),
$$


where

$$
\mathcal{P}(f)(v, \xi)=\left(\begin{array}{c}
-\widetilde{y}_{\mathrm{h}}^{-1}(v)\left(\widetilde{r}_{\mathrm{h}}\left(v+f_{1}(v, \xi)\right)-\widetilde{r}_{\mathrm{h}}(v)-\widetilde{y}_{\mathrm{h}}(v) f_{1}(v, \xi)-\pi_{1} \circ \Gamma_{1}^{u} \circ(\operatorname{Id}+f)(v, \xi)\right) \\
\widetilde{\alpha}_{\mathrm{h}}(v)-\widetilde{\alpha}_{\mathrm{h}}\left(v+f_{1}(v, \xi)\right)-\pi_{3} \circ \Gamma_{1}^{u} \circ(\operatorname{Id}+f)(v, \xi)
\end{array}\right) .
$$

Then, defining the space $\widehat{\mathcal{Z}}_{\nu, \kappa, \delta, \sigma}$ analogously to (178), with only two components $f=\left(f_{1}, f_{2}\right)$ and the same norm, one has

Proposition 5.21. Consider the constants $\kappa_{1}, \delta_{1}$ and $\sigma_{1}$ given by Proposition [5.20 and any $\kappa_{2}>\kappa_{1}$, $\delta_{2}>\delta_{1}$ and $\sigma_{2}<\sigma_{1}$. Then,

- There exists $b_{4}>0$ independent of $G_{0}$ and $\mu$ such that, if $G_{0}$ is large enough, the operator $\mathcal{P}$ has a unique fixed point $f=\left(f_{1}, f_{2}\right) \in \widehat{\mathcal{Z}}_{0, \kappa_{2}, \delta_{2}, \sigma_{2}}$ with

$$
\|f\|_{0, \sigma_{2}} \leq b_{4} \mu G_{0}^{-4} .
$$

- The change $\operatorname{Id}+f$ is the inverse of the restriction of the change given by Theorem 5.16 to the domain $D_{\rho_{2}, \kappa_{1}, \delta_{1}}^{u} \cap \widetilde{D}_{\kappa_{2}, \delta_{2}}$.

- Moreover, the equation (84) defines a formal Fourier series of a generating function which can be written as $T^{u}=T_{0}+T_{1}^{u}$ where $T_{0}$ has been defined in (69) and $T_{1}$ satisfies

$$
\left\|\partial_{v}^{m} \partial_{\xi}^{n} T_{1}^{u}\right\|_{0, \sigma_{2}} \leq K \mu G_{0}^{-4}
$$

for any $0 \leq m+n \leq 1$.

Proof. The first part follows from rewriting Lemma 5.19 in this setting and taking into account that $\left\|\Gamma_{1}^{u}\right\|_{0, \kappa, \sigma} \leq K \mu G_{0}^{-4}$. The second, from the uniqueness statements of the first part and Theorem 5.16.

Finally, the third statement is deduced from equation (84), taking into account that the formal generating function $T^{u}$ was already defined in $D_{\rho_{2}, \kappa_{1}, \delta_{1}}^{u} \cap \widetilde{D}_{\kappa_{2}, \delta_{2}}$ thanks to Theorem 5.10 .

With this proposition we have finished the extension procedure and we have both invariant manifolds parameterized as formal Fourier series, $T^{u, s}$, in the domain $D_{\kappa_{2}, \delta_{2}}$. We summarize this fact in the following theorem.

Theorem 5.22. Let $\kappa_{2}$ and $\delta_{2}$ the constants given by Proposition 5.21. Then, there exist formal Fourier series $T_{1}^{u, s}$ with Fourier coefficients defined in $D_{\kappa_{2}, \delta_{2}}$ (the domain defined in (43)) which are solutions of equation (24) and satisfy

$$
\left\|\partial_{v}^{m} \partial_{\xi}^{n} T_{1}^{u, s}\right\|_{1, \sigma_{2}} \leq b_{5} \mu G_{0}^{-4}
$$

with $0 \leq m+n \leq 1$ and $b_{5}>0$ a constant independent of $\mu$ and $G_{0}$.

Proof. It is enough to join the results of Theorem 5.10 and Proposition 5.21. Indeed, in Theorem 5.10 we have obtained solutions as formal Fourier series of equation (24) of the form $T_{1}^{u, s}=Q_{0}^{u, s}+Q^{u, s}$ in the domains $D_{\rho_{1}, \kappa_{0}, \delta_{0}}^{u, s}$. Using the fact that $D_{\kappa_{2}, \delta_{2}} \subset D_{\rho_{1}, \kappa_{0}, \delta_{0}}^{s}$ and the estimates obtained in Lemma 5.13 and Theorem 5.10 we obtain the desired estimates for $T_{1}^{s}$. For the unstable manifold, we have to recall that $D_{\kappa_{2}, \delta_{2}} \subset D_{\rho_{1}, \kappa_{0}, \delta_{0}}^{s} \cup \widetilde{D}_{\kappa_{2}, \delta_{2}}$. Then, we need also to take into account the results obtained in Proposition 5.21 and recall that since the points $\widetilde{D}_{\kappa_{2}, \delta_{2}}$ are far from the singularities $v= \pm i / 3$, the norms $\|\cdot\|_{1, \sigma_{2}}$ and $\|\cdot\|_{0, \sigma_{2}}$ are equivalent and satisfy $\|\cdot\|_{0, \sigma_{2}} \leq K\|\cdot\|_{1, \sigma_{2}}$.

\section{The difference between the manifolds}

Once we have obtained the parameterization of the invariant manifolds (as formal Fourier series) up to points $\mathcal{O}\left(G_{0}^{-3}\right)$ close to the singularities $v= \pm i / 3$, the next step is to study their difference. It suffices to study the difference between the Fourier coefficients of the generating functions $T^{u, s}$ defined in (23). To this end, we define

$$
\widetilde{\Delta}(v, \xi)=T^{s}(v, \xi)-T^{u}(v, \xi)
$$


Recall that $T^{u, s}(v, \xi)=T_{0}(v, \xi)+T_{1}^{u, s}(v, \xi)$ where $T_{0}$ is defined in (69) and $T_{1}^{u, s}$ are the Fourier series obtained in Theorem 5.22. Therefore, the Fourier coefficients of $\widetilde{\Delta}$ are defined in $D_{\kappa_{2}, \delta_{2}}$. Recall that $\widetilde{\Delta}$ is not a function but a formal Fourier series that does not need to be convergent.

Subtracting equation (24) for $T_{1}^{s}(v, \xi)$ and $T_{1}^{u}(v, \xi)$ considered as equations of formal Fourier series, we can easily see that

$$
\widetilde{\Delta} \in \operatorname{Ker} \widetilde{\mathcal{L}}
$$

where $\widetilde{\mathcal{L}}$ is the differential operator

$$
\widetilde{\mathcal{L}}=(1+A(v, \xi)) \partial_{v}-G_{0}^{3}(1+B(v, \xi)) \partial_{\xi}
$$

with

$$
\begin{aligned}
A(v, \xi)= & \frac{1}{2 \widetilde{y}_{\mathrm{h}}^{2}}\left(\left(\partial_{v} T_{1}^{s}+\partial_{v} T_{1}^{u}\right)-\frac{1}{\widetilde{r}_{\mathrm{h}}^{2}}\left(\partial_{\xi} T_{1}^{s}+\partial_{\xi} T_{1}^{u}\right)\right) \\
B(v, \xi)= & \frac{G_{0}^{-3}}{2 \widetilde{y}_{\mathrm{h}}^{2}}\left(\left(\partial_{v} T_{1}^{s}+\partial_{v} T_{1}^{u}\right)-\frac{1}{\widetilde{r}_{\mathrm{h}}^{2}}\left(\partial_{\xi} T_{1}^{s}+\partial_{\xi} T_{1}^{u}\right)\right) \\
& -\frac{G_{0}^{-3}}{2 \widetilde{r}_{\mathrm{h}}^{2}}\left(\partial_{\xi} T_{1}^{s}+\partial_{\xi} T_{1}^{u}\right) .
\end{aligned}
$$

where $T_{1}^{u, s}$ are the formal Fourier series given by Theorem 5.22 .

Recall that the equation $\widetilde{\mathcal{L}} \widetilde{\Delta}=0$ is stated as an equation for not necessarily convergent Fourier series and not as an equation for functions. The derivatives for Fourier series have been defined in the natural way in (63).

To study the function $\widetilde{\Delta}$ we proceed as in Sau01 (see also GOS10]). That is, we consider a near the identity change of coordinates which conjugates the operator $\widetilde{\mathcal{L}}$ in $(86)$ with the operator $\mathcal{L}$ in $(34)$. Recall that, as we observed in Section 5.5 the composition of formal Fourier series with a near the identity formal transformation is well defined under suitable hypotheses (see Lemma 6.2 below).

The structure of this section goes as follows. First, in Section 6.1 we introduce a functional setting to study the difference between the generating functions. This functional setting is essentially the same as the one considered in Section 5.4 .1 but referred to Fourier series with coefficients defined in $D_{\kappa, \delta}$. In Section 6.2 we straighten the operator $\widetilde{\mathcal{L}}$. Finally, in Section 6.3 , we use this result to prove Theorem 3.2

\subsection{Weighted Fourier norms and Banach spaces}

We devote this section to define Banach spaces for Fourier series with coefficients defined in $D_{\kappa, \delta}$. First, we define the Banach spaces for the Fourier coefficients as

$$
\mathcal{P}_{\nu_{-}, \nu_{+}, \kappa, \delta}=\left\{h: D_{\kappa, \delta} \rightarrow \mathbb{C}: \text { analytic, }\|h\|_{\nu_{-}, \nu_{+}}<\infty\right\},
$$

where

$$
\|h\|_{\nu_{-}, \nu_{+}}=\sup _{v \in D_{\kappa, \delta}}\left|(v-i / 3)^{\nu_{+}}(v+i / 3)^{\nu_{-}} h(v)\right| .
$$

Note that these definitions are the same as (59) and (60) but for functions defined in $D_{\kappa, \delta}$ instead of $D_{\rho, \kappa, \delta}^{u}$. Now we define the Banach space for Fourier series

$$
\mathcal{Q}_{\nu, \kappa, \delta, \sigma}=\left\{h(v, \xi)=\sum_{\ell \in \mathbb{Z}} h^{[\ell]}(v) e^{i \ell \xi}: h^{[\ell]} \in \mathcal{P}_{\nu+\ell / 2, \nu-\ell / 2, \kappa, \delta},\|h\|_{\nu, \sigma}<\infty\right\} .
$$

where

$$
\|h\|_{\nu, \sigma}=\sum_{\ell \in \mathbb{Z}}\left\|h^{[\ell]}\right\|_{\nu+\ell / 2, \nu-\ell / 2} e^{|\ell| \sigma} .
$$

The Banach space $\mathcal{Q}_{\nu, \kappa, \delta, \sigma}$ satisfies the algebra properties stated in Lemma 5.9. Therefore, from now on in this section, we will refer to this lemma understanding the properties stated in it as properties referred 
to elements of $\mathcal{Q}_{\nu, \kappa, \delta, \sigma}$ instead of elements of $\mathcal{Y}_{\nu, \kappa, \delta, \sigma}$. Moreover, in the present section we will need to take derivatives of and compose Fourier series. To this end we state the following two technical lemmas, which are equivalent to Lemmas 5.14 and 5.15

Lemma 6.1. Fix constants $\sigma^{\prime}<\sigma, \kappa^{\prime}>\kappa$ and $\delta^{\prime}>\delta$ and take $h \in \mathcal{Q}_{\nu, \kappa, \delta, \sigma}$. Its derivatives, as defined in (63), satisfy

- $\partial_{v}^{m} h \in \mathcal{Q}_{\nu, \kappa^{\prime}, \delta^{\prime}, \sigma^{\prime}}$ and

$$
\left\|\partial_{v}^{m} h\right\|_{\nu, \sigma^{\prime}} \leq\left(\frac{\kappa^{\prime}}{\kappa}\right)^{\nu} \frac{G_{0}^{3 m} m !}{\left(\kappa^{\prime}-\kappa\right)^{m}}\|h\|_{\nu, \sigma}
$$

- $\partial_{\xi} h \in \mathcal{Q}_{\nu, \kappa^{\prime}, \delta^{\prime}, \sigma^{\prime}}$ and

$$
\left\|\partial_{\xi} h\right\|_{\nu, \sigma^{\prime}} \leq \frac{1}{\sigma-\sigma^{\prime}}\|h\|_{\nu, \sigma}
$$

Lemma 6.2. We define the formal composition of formal Fourier series

$$
h(v+g(v, \xi), \xi)=\sum_{m=0}^{\infty} \frac{1}{m !} \partial_{v}^{m} h(v, \xi) g^{m}(v, \xi) .
$$

Fix constants $\sigma^{\prime}<\sigma, \kappa^{\prime}>\kappa$ and $\delta^{\prime}>\delta$. Let $\kappa^{\prime}-\kappa>\eta>0$. Then,

- If $h \in \mathcal{Q}_{\nu, \kappa, \delta, \sigma}, g \in \mathcal{Q}_{0, \kappa^{\prime}, \delta^{\prime}, \sigma^{\prime}}$ and $\|g\|_{0, \sigma^{\prime}} \leq \eta G_{0}^{-3}$ we have that $X(v, \xi)=h(v+g(v, \xi)$, $\xi)$ satisfies $X \in \mathcal{Y}_{\nu, \kappa^{\prime}, \delta^{\prime}, \sigma^{\prime}}$ and

$$
\|X\|_{\nu, \sigma^{\prime}} \leq\left(\frac{\kappa^{\prime}}{\kappa}\right)^{\nu}\left(1-\frac{\eta}{\kappa^{\prime}-\kappa}\right)^{-1}\|h\|_{\nu, \sigma} .
$$

Moreover, if $\left\|g_{1}\right\|_{0, \sigma},\left\|g_{2}\right\|_{0, \sigma} \leq \eta G_{0}^{-3}$, then $Y(v, \xi)=h\left(v+g_{2}(v, \xi), \xi\right)-h\left(v+g_{1}(v, \xi), \xi\right)$ satisfies

$$
\|Y\|_{\nu, \sigma^{\prime}} \leq \frac{G_{0}^{3}}{\kappa^{\prime}-\kappa}\left(\frac{\kappa^{\prime}}{\kappa}\right)^{\nu}\left(1-\frac{\eta}{\kappa^{\prime}-\kappa}\right)^{-2}\|h\|_{\nu, \sigma}\left\|g_{2}-g_{1}\right\|_{0, \sigma} .
$$

- If $\partial_{v} h \in \mathcal{Q}_{\nu, \kappa, \delta, \sigma}, g_{1}, g_{2} \in \mathcal{Q}_{0, \kappa^{\prime}, \delta^{\prime}, \sigma^{\prime}}$ and $\left\|g_{1}\right\|_{0, \sigma^{\prime}},\left\|g_{2}\right\|_{0, \sigma^{\prime}} \leq \eta G_{0}^{-3}$ we have that $Y \in \mathcal{Y}_{\nu, \kappa^{\prime}, \delta^{\prime}, \sigma^{\prime}}$ and

$$
\|Y\|_{\nu, \sigma^{\prime}} \leq\left(\frac{\kappa^{\prime}}{\kappa}\right)^{\nu} \frac{1}{1-\frac{\eta}{\kappa^{\prime}-\kappa}}\left\|\partial_{v} h\right\|_{\nu, \sigma}\left\|g_{2}-g_{1}\right\|_{0, \sigma^{\prime}} .
$$

\subsection{Straightening the operator $\widetilde{\mathcal{L}}$}

Once we have defined the Banach space $\mathcal{Q}_{\nu, \kappa, \delta, \sigma}$ we can show that the operator $\widetilde{\mathcal{L}}$ can be straightened, as stated in the following theorem.

Theorem 6.3. Let $\sigma_{2}, \kappa_{2}$ and $\delta_{2}$ be the constants given by Theorem 5.22. Let $\sigma_{3}\left\langle\sigma_{2}, \kappa_{3}>\kappa_{2}\right.$ and $\delta_{3}>\delta_{2}$ be fixed. Then, for $G_{0}$ big enough, there exists a (not necessarily convergent) Fourier series $\mathcal{C} \in \mathcal{Q}_{0, \kappa_{3}, \delta_{3}, \sigma_{3}}$ satisfying

$$
\|\mathcal{C}\|_{0, \sigma_{3}} \leq b_{6} \mu G_{0}^{-4}
$$

with $b_{6}>0$ a constant independent of $\mu$ and $G_{0}$, such that

$$
\Delta(w, \xi)=\widetilde{\Delta}(w+\mathcal{C}(w, \xi), \xi),
$$

where $\widetilde{\Delta}$ is the function defined in (85), is well defined and satisfies that $\Delta \in$ Ker $\mathcal{L}$ and $\mathcal{L}$ is the operator defined in (34).

Moreover, one can choose $\mathcal{C}$ to satisfy

$$
\mathcal{C}(-w,-\xi)=-\mathcal{C}(w, \xi),
$$

and therefore, by (27), one has that

$$
\Delta(-w,-\xi)=\Delta(w, \xi)
$$


To prove this theorem we need a change of variables $v=w+\mathcal{C}(w, \xi)$ such that $\widetilde{\Delta} \in \operatorname{Ker} \widetilde{\mathcal{L}}$ if and only if $\Delta \in \operatorname{Ker} \mathcal{L}$. This fact is equivalent to look for a Fourier series $\mathcal{C}$ which is solution of the equation

$$
\mathcal{L}(\mathcal{C})=\mathcal{K}(\mathcal{C})
$$

where

$$
\mathcal{K}(h)(w, \xi)=\left.\frac{A(v, \xi)-B(v, \xi)}{1+B(v, \xi)}\right|_{v=w+h(w, \xi)}
$$

and $A$ and $B$ are given in 87 .

We devote the rest of this section to obtain a solution of this equation. Let us point out that the fraction in the right hand side, since it involves formal Fourier series, is understood as

$$
\frac{1}{1+B(v, \xi)}=\sum_{\ell \geq 0}(-B(v, \xi))^{\ell}
$$

The solution of equation (89) is found through a fixed point argument. As we will see in Lemma 6.6 the functions $A$ and $B$ inherit the symmetry properties of the manifolds $T^{u}$ and $T^{s}$ in (27). To obtain a symmetric solution $\mathcal{C}$ of the equation (89), we need a suitable left inverse of the operator $\mathcal{L}$ in the domain $D_{\kappa, \delta}$, which is a slight modification of the operator $\widetilde{\mathcal{G}}$ in (66) acting on the Fourier coefficients and is defined as

$$
\widetilde{\mathcal{G}}(h)(v, \xi)=\sum_{\ell \in \mathbb{Z}} \widetilde{\mathcal{G}}(h)^{[\ell]}(v) e^{i \ell \xi},
$$

where its Fourier coefficients are given by

$$
\begin{array}{ll}
\widetilde{\mathcal{G}}(h)^{[\ell]}(v)=\int_{v_{2}}^{v} e^{i \ell G_{0}^{3}(v-t)} h^{[\ell]}(t) d t & \text { for } \ell<0 \\
\widetilde{\mathcal{G}}(h)^{[0]}(v)=\int_{v^{*}}^{v} h^{[0]}(t) d t-\frac{1}{2} \int_{v^{*}}^{v_{2}} h^{[0]}(t) d t-\frac{1}{2} \int_{v^{*}}^{\bar{v}_{2}} h^{[0]}(t) d t & \\
\widetilde{\mathcal{G}}(h)^{[\ell]}(v)=\int_{\bar{v}_{2}}^{v} e^{i \ell G_{0}^{3}(v-t)} h^{[\ell]}(t) d t & \text { for } \ell>0 .
\end{array}
$$

Here $v_{2}=i\left(1 / 3-\kappa G_{0}^{-3}\right)$ is the top vertex of the domain $D_{\kappa, \delta}, \bar{v}_{2}$ is its conjugate, which corresponds to the bottom vertex of the domain $D_{\kappa, \delta}$ and $v^{*}$ is the left endpoint of $D_{\kappa, \delta} \cap \mathbb{R}$.

Lemma 6.4. The operator $\widetilde{\mathcal{G}}$ in (90) satisfies that if $h \in \mathcal{Q}_{\nu, \kappa, \delta, \sigma}$ for some $\nu \in(0,1)$, then $\widetilde{\mathcal{G}}(h) \in \mathcal{Q}_{0, \kappa, \delta, \sigma}$ and

$$
\|\widetilde{\mathcal{G}}(h)\|_{0, \sigma} \leq K\|h\|_{\nu, \sigma} .
$$

Moreover,

- If $h$ is a real-analytic Fourier series, that is

$$
h^{[\ell]}(\bar{v})=\overline{h^{[-\ell]}(v)},
$$

then so is $\widetilde{\mathcal{G}}(h)$.

- If $h$ satisfies $h(-v,-\xi)=h(v, \xi)$, one has that

$$
\widetilde{\mathcal{G}}(h)(-v,-\xi)=-\widetilde{\mathcal{G}}(h)(v, \xi) .
$$

Proof. The first part is proven as Lemma 8.3 of GOS10. The real-analyticity property is straightforward. To prove the symmetry property the first observation is that, if $h$ is even and taking into account that $\bar{v}_{2}=-v_{2}$, one has that for $\ell \neq 0$,

$$
\widetilde{\mathcal{G}}(h)^{[-\ell]}(-v)=-\widetilde{\mathcal{G}}(h)^{[\ell]}(v) .
$$


Therefore, one just needs to see that $\widetilde{\mathcal{G}}(h)^{[0]}(v)$ is an odd function. To this end, it is enough to check that

$$
f(v)=\widetilde{\mathcal{G}}(h)^{[0]}(-v)+\widetilde{\mathcal{G}}(h)^{[0]}(v)
$$

satisfies, wherever it makes sense, $f^{\prime}(v)=0$ and $f\left(v_{2}\right)=0$.

We look for a fixed point of the operator

$$
\widetilde{\mathcal{K}}=\widetilde{\mathcal{G}} \circ \mathcal{K}
$$

in the space $\mathcal{Q}_{0, \kappa, \delta, \sigma}$. Theorem 6.3 is a straightforward consequence of next proposition.

Proposition 6.5. Fix $\sigma_{3}<\sigma_{2}, \kappa_{3}>\kappa_{2}$ and $\delta_{3}>\delta_{2}$. Then, there exists a constant $b_{6}>0$ such that for $G_{0}$ big enough the operator $\widetilde{\mathcal{K}}$ is well defined from $B\left(b_{6} \mu G_{0}^{-4}\right) \subset \mathcal{Q}_{0, \kappa_{3}, \delta_{3}, \sigma_{3}}$ to itself and it is contractive. Therefore, it has a unique fixed point $\mathcal{C}$ in this ball, which is a real-analytic formal Fourier series and satisfies the symmetry condition $\mathcal{C}(-v,-\xi)=-\mathcal{C}(v, \xi)$.

To prove the proposition, we first state the following technical lemma, whose proof follows easily from Theorem 5.22 and the properties of the homoclinic parameterization given in Corollary 4.3 and (16).

Lemma 6.6. The functions $A$ and $B$ defined in (87) satisfy that $A \in \mathcal{Q}_{1 / 2, \kappa_{2}, \delta_{2}, \sigma_{2}}$ and $B \in \mathcal{Q}_{1 / 2, \kappa_{2}, \delta_{2}, \sigma_{2}}$ and the symmetry properties

$$
A(-v,-\xi)=A(v, \xi) \quad \text { and } \quad B(-v,-\xi)=B(v, \xi)
$$

Moreover,

$$
\begin{aligned}
\|A\|_{1 / 2, \sigma_{2}} & \leq K \mu G_{0}^{-4} \\
\|B\|_{1 / 2, \sigma_{2}} & \leq K \mu G_{0}^{-4} .
\end{aligned}
$$

Proof of Proposition 6.5. First we see that if we fix any $K>0$, then for $G_{0}$ big enough, the operator $\widetilde{\mathcal{K}}$ is well defined from $B\left(K \mu G_{0}^{-4}\right) \subset \mathcal{Q}_{0, \kappa_{1}, \delta, \sigma_{1}}$ to $\mathcal{Q}_{0, \kappa_{3}, \delta_{3}, \sigma_{3}}$. Indeed, take $h \in B\left(K \mu G_{0}^{-4}\right) \subset \mathcal{Q}_{0, \kappa_{3}, \delta_{3}, \sigma_{3}}$. Then, applying Lemmas 6.2 and 6.6 with $\eta=K \mu G_{0}^{-1}$ and taking $G_{0}$ large enough, one can easily see that $\mathcal{K}(h) \in \mathcal{Q}_{1 / 2, \kappa_{3}, \delta_{3}, \sigma_{3}}$. Then, applying Lemma 6.4, we have that $\widetilde{\mathcal{K}}(h)=\widetilde{\mathcal{G}} \circ \mathcal{K}(h) \in \mathcal{Q}_{0, \kappa_{1}, \delta, \sigma_{1}}$.

Once we know that the operator is well defined, we prove that it is contractive in a certain ball. As a first step we look for bounds for $\widetilde{\mathcal{K}}(0)$. By Lemma 6.6. one can easily see that

$$
\|\mathcal{K}(0)\|_{1 / 2, \sigma_{3}} \leq K \mu G_{0}^{-4}
$$

Then, applying Lemma 6.4 we have that there exists a constant $b_{6}>0$ such that

$$
\|\widetilde{\mathcal{K}}(0)\|_{0, \sigma_{3}} \leq \frac{b_{6}}{2} \mu G_{0}^{-4} .
$$

Finally, we take $h_{1}, h_{2} \in B\left(b_{6} \mu G_{0}^{-4}\right) \subset \mathcal{Q}_{0, \kappa_{3}, \delta_{3}, \sigma_{3}}$ and we prove that $\widetilde{\mathcal{K}}$ is contractive. To this end, we first bound $\mathcal{K}\left(h_{2}\right)-\mathcal{K}\left(h_{1}\right)$. Using Lemma 6.2 and the estimates obtained in Lemma 6.6, one can easily see that

$$
\left\|\mathcal{K}\left(h_{2}\right)-\mathcal{K}\left(h_{1}\right)\right\|_{1 / 2, \sigma_{3}} \leq K \mu G_{0}^{-1}\left\|h_{2}-h_{1}\right\|_{0, \sigma_{3}} .
$$

Therefore, applying Lemma 6.4, we obtain that

$$
\left\|\widetilde{\mathcal{K}}\left(h_{2}\right)-\widetilde{\mathcal{K}}\left(h_{1}\right)\right\|_{0, \sigma_{3}} \leq K \mu G_{0}^{-1}\left\|h_{2}-h_{1}\right\|_{0, \sigma_{3}} .
$$

Thus, this gives the existence of a unique fixed point $\mathcal{C}$ of the operator $\widetilde{\mathcal{K}}$. Furthermore, by Lemmas 6.4 and 6.6. one has that $\widetilde{\mathcal{K}}(h)(-v,-\xi)=-\widetilde{\mathcal{K}}(h)(v, \xi)$ if $h$ satisfies $h(-v,-\xi)=-h(v, \xi)$ and therefore the fixed point $\mathcal{C}$ satisfies the required symmetry property. Reasoning analogously one also obtains real-analyticity. This completes the proof of the proposition. 


\subsection{Estimates for the difference between invariant manifolds}

We devote this section to complete the proof of Theorem 3.2, that is, to prove that the generalized Poincaré function (28) gives the first order of the difference between the manifolds. As a first step, we show that not necessarily convergent Fourier series $\Psi \in \mathcal{Q}_{0, \kappa, \delta, \sigma}$ such that $\Psi \in \operatorname{Ker} \mathcal{L}$ define functions for real values of the variables which have exponentially small bounds in $G_{0}$.

Lemma 6.7. Fix $\kappa>0, \delta>0$ and $\sigma>0$. Let us consider a formal Fourier series $\Psi \in \mathcal{Q}_{0, \kappa, \delta, \sigma}$ such that $\Psi \in \operatorname{Ker} \mathcal{L}$. Then, the Fourier series $\Psi(v, \xi)$

- is of the form

$$
\Psi(v, \xi)=\sum_{\ell \in \mathbb{Z}} \Psi^{[\ell]}(v) e^{i \ell \xi}=\sum_{\ell \in \mathbb{Z}} \Lambda^{[\ell]} e^{i \ell\left(G_{0}^{3} v+\xi\right)}
$$

for certain constants $\Lambda^{[\ell]} \in \mathbb{C}$ and

- defines a function for $v \in D_{\kappa, \delta} \cap \mathbb{R}$ and $\xi \in \mathbb{T}$, which satisfies that

$$
\begin{gathered}
\left|\Psi^{[\ell]}(v)\right| \leq \sup _{u \in D_{\kappa, \delta} \cap D_{\rho, \kappa, \delta}^{u}}\left|\Psi^{[\ell]}(u)\right| K^{|\ell|} e^{-\frac{|\ell| G_{0}^{3}}{3}} \\
\left|\partial_{v} \Psi^{[\ell]}(v)\right| \leq \sup _{u \in D_{\kappa, \delta} \cap D_{\rho, \kappa, \delta}^{u}}\left|\Psi^{[\ell]}(u)\right| K^{|\ell|} G_{0}^{3} e^{-\frac{|\ell| G_{0}^{3}}{3}} \\
\left|\partial_{v}^{2} \Psi^{[\ell]}(v)\right| \leq \sup _{u \in D_{\kappa, \delta} \cap D_{\rho, \kappa, \delta}^{u}}\left|\Psi^{[\ell]}(u)\right| K^{|\ell|} G_{0}^{6} e^{-\frac{|\ell| G_{0}^{3}}{3}} .
\end{gathered}
$$

Proof. The fact that $\Psi \in \operatorname{Ker} \mathcal{L}$ implies that each Fourier coefficient $\Psi^{[\ell]}$ satisfies

$$
\partial_{v} \Psi^{[\ell]}(v)-i \ell G_{0}^{3} \Psi^{[\ell]}(v)=0
$$

and therefore, there exist constants $\Lambda^{[\ell]}$ such that

$$
\Psi^{[\ell]}(v)=\Lambda^{[\ell]} e^{i \ell G_{0}^{3} v} .
$$

Evaluating this equality at the top vertex $v_{2}=i\left(1 / 3-\kappa G_{0}^{-3}\right)$ of $D_{\kappa, \delta}$ for $\ell<0$ and at the bottom vertex $\bar{v}_{2}=-i\left(1 / 3-\kappa G_{0}^{-3}\right)$ for $\ell>0$, we obtain that

$$
\left|\Lambda^{[\ell]}\right| \leq K^{|\ell|} \max \left\{\left|\Psi^{[\ell]}\left(v_{2}\right)\right|,\left|\Psi^{[\ell]}\left(\bar{v}_{2}\right)\right|\right\} e^{-|\ell| \frac{G_{0}^{3}}{3}} .
$$

This implies that for $v \in D_{\kappa, \delta} \cap \mathbb{R}$

$$
\left|\Psi^{[\ell]}(v)\right|=\left|\Lambda^{[\ell]}\right| \leq \sup _{u \in D_{\kappa, \delta} \cap D_{\rho, \kappa, \delta}^{u}}\left|\Psi^{[\ell]}(u)\right| K^{|\ell|} e^{-|\ell| \frac{G_{0}^{3}}{3}} .
$$

Moreover, since $\Psi \in \mathcal{Q}_{0, \kappa, \delta, \sigma}$, we have that

$$
\left|\Psi^{[\ell]}(v)\right| \leq\|\Psi\|_{0, \sigma}\left(K G_{0}^{3}\right)^{\frac{|\ell|}{2}} e^{-|\ell| \frac{G_{0}^{3}}{3}} .
$$

From this bound one has that, for $v \in D_{\kappa, \delta} \cap \mathbb{R}$ and $\xi \in \mathbb{T}$, the Fourier series of $\Psi$ is convergent and therefore it defines an analytic function. Finally, taking derivatives one can easily prove the bounds for $\partial_{v} \Psi^{[\ell]}$ and $\partial_{v}^{2} \Psi^{[\ell]}$. 
We use Lemma 6.7 to prove that (28) gives the first order of the difference between the manifolds. First let us observe that, since by Theorem 6.3 the Fourier series $\Delta$ in (88) is symmetric, real-analytic and satisfies $\Delta \in \operatorname{Ker} \mathcal{L}$, it is of the form

$$
\Delta(w, \xi)=\Gamma^{[0]}+2 \sum_{\ell \in \mathbb{Z}} \Gamma^{[\ell]} \cos \ell\left(G_{0}^{3} w+\xi\right) .
$$

for certain coefficients $\Gamma^{[\ell]} \in \mathbb{R}$.

A direct application of Lemma 6.7. provides exponentially small bounds for $\Delta$. Nevertheless, our goal is not give bounds but to prove that the function $L$ in (28) is the main term in $\Delta$. Thus, we define the formal Fourier series

$$
\mathcal{E}(w, \xi)=\Delta(w, \xi)-L(w, \xi),
$$

Note that, by (36), $L$ can be expressed as a formal Fourier series with coefficients in the domain $D_{\kappa, \delta} \cap$ $D_{\rho, \kappa, \delta}^{u}$ as

$$
L=\left(Q_{0}^{s}-Q_{0}^{u}\right)-\left(L_{1}^{s}-L_{1}^{u}\right),
$$

where $Q_{0}^{u}$ and $L_{1}^{u}$ are the Fourier series defined in (39) and (52), respectively, and $Q_{0}^{s}$ and $L_{1}^{s}$ are the analogous Fourier series referred to the stable manifold.

Lemma 6.8. Consider the constants $\kappa_{3}$ and $\delta_{3}$ defined in Theorem 6.3. Then, for $(w, \xi) \in\left(D_{\kappa_{3}, \delta_{3}} \cap\right.$ $\mathbb{R}) \times \mathbb{T}$, the Fourier series $\mathcal{E}$ defines an analytic function, which satisfies

$$
|\mathcal{E}(w, \xi)-E| \leq K \mu^{2}(1-2 \mu) G_{0}^{-2} e^{-\frac{G_{0}^{3}}{3}}+K G_{0}^{-1 / 2} \mu^{2} e^{-\frac{2 G_{0}^{3}}{3}},
$$

where $E \in \mathbb{R}$ is a constant, and for $0<m+n \leq 2$,

$$
\left|\partial_{v}^{m} \partial_{\xi}^{n} \mathcal{E}(w, \xi)\right| \leq K \mu^{2}(1-2 \mu) G_{0}^{-2+3 m} e^{-\frac{G_{0}^{3}}{3}}+K G_{0}^{-1 / 2+3 m} \mu^{2} e^{-\frac{2 G_{0}^{3}}{3}} .
$$

Proof. To proof the lemma it is enough to point out that $\mathcal{E} \in \operatorname{Ker} \mathcal{L}$ and then apply Lemma 6.7. We use the expression of $L$ given in (92). By Theorem 6.3. we have that $\Delta \in \operatorname{Ker} \mathcal{L}$. Moreover, $\mathcal{L}\left(Q_{0}^{*}\right)=\widehat{U}_{0}$ for both $*=u$ and $*=s$ and therefore $\mathcal{L}\left(Q_{0}^{s}-Q_{0}^{u}\right)=0$. Reasoning analogously, $\mathcal{L}\left(L_{1}^{s}-L_{1}^{u}\right)=0$. Then, to apply Lemma 6.7 it only suffices to bound $\|\mathcal{E}\|_{0, \sigma_{3}}$ in the domain $D_{\kappa_{3}, \delta_{3}} \cap D_{\rho_{2}, \kappa_{3}, \delta_{3}}^{u}$. To this end, we split $\mathcal{E}$ as

$$
\mathcal{E}=\mathcal{E}_{1}^{s}-\mathcal{E}_{1}^{u}+\mathcal{E}_{2}
$$

where

$$
\begin{aligned}
& \mathcal{E}_{1}^{*}=T_{1}^{*}-Q_{0}^{*}-L_{1}^{*} \\
& \mathcal{E}_{2}=\Delta-\widetilde{\Delta} .
\end{aligned}
$$

We start by bounding $\left\|\mathcal{E}_{1}^{u}\right\|_{1, \sigma_{3}}$. By the definition of $Q^{u}$ in (40), we have that $\mathcal{E}_{1}^{u}=Q^{u}-L_{1}^{u}$. Then, applying formula (65), we obtain

$$
\left\|\mathcal{E}_{1}^{u}\right\|_{1, \sigma_{3}} \leq K \mu^{2} G_{0}^{-8} \text {. }
$$

Then, applying Lemma 5.9, we obtain that

$$
\left\|\mathcal{E}_{1}^{u}\right\|_{0, \sigma_{3}} \leq K \mu^{2} G_{0}^{-5}
$$

The bound for $\mathcal{E}_{1}^{s}$ is analogous. To bound $\mathcal{E}_{2}$, recall that by the definition of $\Delta$ in (88), it can be written as

$$
\mathcal{E}_{2}(w, \xi)=\widetilde{\Delta}(w+\mathcal{C}(w, \xi), \xi)-\widetilde{\Delta}(w, \xi)
$$

From Lemma [5.13, to bound $Q_{0}^{*}$, Theorem [5.10, to bound $Q^{*}$, and Lemma 5.9] we know that $\partial_{v} \widetilde{\Delta} \in$ $\mathcal{Q}_{3 / 2, \kappa_{3}, \delta_{3}, \sigma_{3}}$ and satisfies $\left\|\partial_{v} \widetilde{\Delta}\right\|_{3 / 2, \sigma_{3}} \leq K \mu G_{0}^{-4}$. Therefore, applying Lemma 6.2 and using the estimates for $\mathcal{C}$ given in Theorem 6.3 we obtain that

$$
\left\|\mathcal{E}_{2}\right\|_{3 / 2, \sigma_{3}} \leq\left\|\partial_{v} \widetilde{\Delta}\right\|_{3 / 2, \sigma_{2}}\|\mathcal{C}\|_{0, \sigma_{3}} \leq K \mu^{2} G_{0}^{-8} .
$$


Applying Lemma 5.9, we obtain that

$$
\left\|\mathcal{E}_{2}\right\|_{0, \sigma_{3}} \leq K \mu^{2} G_{0}^{-7 / 2}
$$

and thus we can conclude that

$$
\|\mathcal{E}\|_{0, \sigma_{3}} \leq K \mu^{2} G_{0}^{-7 / 2}
$$

which implies

$$
\left|\mathcal{E}^{[\ell]}(v)\right| \leq \mu^{2}\left(K G_{0}\right)^{-\frac{7}{2}+\frac{3}{2}|\ell|} .
$$

Recall that, when $\mu=1 / 2$, the Hamiltonian (17) is $\pi$-periodic. It can be easily seen that this fact implies that $\mathcal{E}$ is $\pi$-periodic in $\xi$ and therefore, when $\mu=1 / 2$, it satisfies that $\mathcal{E}^{[\ell]}=0$ for any odd $\ell$. On the other hand, the function $\mathcal{E}$ depends analytically on $\mu$ and therefore one can apply the Schwarz Lemma to see that for any odd $\ell$,

$$
\left|\mathcal{E}^{[\ell]}(v)\right| \leq \mu^{2}(1-2 \mu)\left(K G_{0}\right)^{-\frac{7}{2}+\frac{3}{2}|\ell|} .
$$

Applying now Lemma 6.7, we obtain that for $w \in D_{\kappa_{3}, \delta_{3}} \cap \mathbb{R}$,

$$
\begin{array}{ll}
\left|\mathcal{E}^{[\ell]}(v)\right| \leq \mu^{2}(1-2 \mu)\left(K G_{0}\right)^{-\frac{7}{2}+\frac{3}{2}|\ell|} e^{-\frac{|\ell| G_{0}^{3}}{3}} & \text { for odd }|\ell| \\
\left|\mathcal{E}^{[\ell]}(v)\right| \leq \mu^{2}\left(K G_{0}\right)^{-\frac{7}{2}+\frac{3}{2}|\ell|} e^{-\frac{|\ell| G_{0}^{3}}{3}} & \text { for even }|\ell|
\end{array}
$$

and analogous bounds for the derivatives of the Fourier coefficients. Defining $E=\mathcal{E}^{[0]}$ and summing up the odd and even Fourier coefficients of $\mathcal{E}$ one obtains the bounds stated in Lemma 6.8.

Now it only remains to go back to the original variables $(v, \xi)$. This is summarized in the next lemma, from which the proof of Theorem 3.2 follows. Recall that now we are taking real values of the variables and therefore all the objects we are dealing with are functions and not only formal Fourier series.

Lemma 6.9. Consider the functions $\widetilde{\Delta}$ defined in (85) and $L$ defined in (28) and the constants $\kappa_{3}$ and $\delta_{3}$ given by Theorem 6.3. Fix $\kappa_{4}>\kappa_{3}$ and $\delta_{4}>\delta_{3}$. Then, for $(v, \xi) \in\left(D_{\kappa_{4}, \delta_{4}} \cap \mathbb{R}\right) \times \mathbb{T}$,

$$
|\widetilde{\Delta}(v, \xi)-L(v, \xi)-E| \leq K \mu^{2}(1-2 \mu) G_{0}^{-2} e^{-\frac{G_{0}^{3}}{3}}+K G_{0}^{-1 / 2} \mu^{2} e^{-\frac{2 G_{0}^{3}}{3}}
$$

for certain $E \in \mathbb{R}$, and for $0<m+n \leq 2$,

$$
\left|\partial_{v}^{m} \partial_{\xi}^{n} \widetilde{\Delta}(v, \xi)-\partial_{v}^{m} \partial_{\xi}^{n} L(v, \xi)\right| \leq K \mu^{2}(1-2 \mu) G_{0}^{-2+3 m} e^{-\frac{G_{0}^{3}}{3}}+K G_{0}^{-1 / 2+3 m} \mu^{2} e^{-\frac{2 G_{0}^{3}}{3}} .
$$

Proof. It is enough to consider the inverse of the change of coordinates $(v, \xi)=(w+\mathcal{C}(w, \xi), \xi)$ obtained in Theorem 6.3 Recall that now we are interested in real values of the variables. Nevertheless, since we need to apply Cauchy estimates we consider a small complex neighborhood of $\left(D_{\kappa_{3}, \delta_{3}} \cap \mathbb{R}\right) \times \mathbb{T}$. Then, shrinking slightly the domain, one can easily obtain the inverse change by means of a fixed point argument. It is of the form $(w, \xi)=(v+\Upsilon(v, \xi), \xi)$ and $\Upsilon$ satisfies $\|\Upsilon\|_{\infty} \leq K \mu G_{0}^{-4}$. Applying Cauchy estimates we also know that for real values of the variables $\left\|\partial_{w} \Upsilon\right\|_{\infty} \leq K \mu G_{0}^{-4}$ and $\left\|\partial_{w}^{2} \Upsilon\right\|_{\infty} \leq K \mu G_{0}^{-4}$. Applying the change of coordinates to the bounds obtained in Lemma 6.8 and using the bounds obtained for $\Upsilon$ and its derivatives, Lemma 6.9 easily follows.

Proof of Theorem [2.3. Due to the form of $\Delta$ in (91), one has that

$$
\Delta(w, \xi)=\Gamma\left(G_{0}^{3} w+\xi\right)
$$

Moreover, it is clear that $\Gamma^{\prime}(\pi k)=\Gamma^{\prime \prime \prime}(\pi k)=0$ for any $k \in \mathbb{Z}$. On the other hand, by Lemma 6.8 and Proposition 3.1, on has that

$$
\begin{aligned}
\Gamma^{\prime \prime}(z)= & 2 \sqrt{\pi} \mu(1-\mu) G_{0}^{-3 / 2} e^{-\frac{G_{0}^{3}}{3}}\left[\frac{1-2 \mu}{4 \sqrt{2}} \cos z\left(1+\mathcal{O}\left(G_{0}^{-1 / 2}\right)\right)+8 G_{0}^{2} e^{-\frac{G_{0}^{3}}{3}} \cos 2 z\left(1+\mathcal{O}\left(G_{0}^{-1 / 2}\right)\right)\right] \\
& +\mathcal{O}\left(\mu G_{0}^{3 / 2} e^{-G_{0}^{3}}\right) .
\end{aligned}
$$


Therefore, for any odd $k \in \mathbb{Z}$, the equation $\Gamma^{\prime \prime}(k \pi)=0$ defines a curve $\eta$ in the parameter plane $\left(\mu, G_{0}\right)$, which is of the form

$$
\mu=\mu^{*}\left(G_{0}\right)=\frac{1}{2}-16 \sqrt{2} G_{0}^{2} e^{-\frac{G_{0}^{3}}{3}}\left(1+\mathcal{O}\left(G_{0}^{1 / 2}\right)\right) .
$$

Moreover, for $\left(\mu, G_{0}\right) \in \eta$, one has that $\Gamma^{(i v)}(k \pi) \neq 0$. This implies that all partial derivatives up to order three of the function $\Delta$ are zero and the fourth are not whenever $G_{0}^{3} w+\xi=k \pi$ for odd $k$.

Now, for $G_{0}$ big enough, we can choose $k \in \mathbb{Z}$, odd, such that, for any $\xi \in \mathbb{T}$, one has that $w_{k}=$ $G_{0}^{-3}(k \pi-\xi) \in D_{\kappa_{3}, \delta_{3}} \cap \mathbb{R}$. Therefore, taking into account the relation between $\Delta$ and $\widetilde{\Delta}$ in (88) , at the point $\left(v_{k}, \xi\right)=\left(w_{k}+\mathcal{C}\left(w_{k}, \xi\right), \xi\right)$, all the partial derivatives up to order three of the function $\widetilde{\Delta}$ vanish and the fourth one does not.

To finish the proof of Theorem 2.3, it only remains to go back to the parameterizations (19) of the stable and unstable invariant curves $\gamma^{u, s}$ of the Poincaré map (18). They are defined by formula (29). Then, at the point $v=v_{k}$, one can easily see that

$$
\begin{aligned}
& \partial_{v}\left(Y_{\phi_{0}}^{s}\left(v_{k} ; \mu^{*}\left(G_{0}\right), G_{0}\right)-Y_{\phi_{0}}^{u}\left(v_{k} ; \mu^{*}\left(G_{0}\right), G_{0}\right)\right)=0 \\
& \partial_{v}^{2}\left(Y_{\phi_{0}}^{s}\left(v_{k} ; \mu^{*}\left(G_{0}\right), G_{0}\right)-Y_{\phi_{0}}^{u}\left(v_{k} ; \mu^{*}\left(G_{0}\right), G_{0}\right)\right)=0 \\
& \partial_{v}^{3}\left(Y_{\phi_{0}}^{s}\left(v_{k} ; \mu^{*}\left(G_{0}\right), G_{0}\right)-Y_{\phi_{0}}^{u}\left(v_{k} ; \mu^{*}\left(G_{0}\right), G_{0}\right)\right) \neq 0 .
\end{aligned}
$$

Therefore, the invariant curves $\gamma^{u, s}$ have a cubic homoclinic tangency.

Let us emphasize that, for $\left(\mu, G_{0}\right) \in \eta$ and any even $k, \Gamma^{\prime \prime}(k \pi) \neq 0$. This implies that, at the corresponding homoclinic points, the invariant curves $\gamma^{u, s}$ intersect transversally.

\section{A Computation of the function L: proof of Proposition 3.1}

We devote this appendix to give the properties of the function $L$ in (28) stated in Proposition 3.1 . We first state an auxiliary lemma, which gives some properties of the potential $V$ in (11).

Lemma A.1. The Fourier coefficients of the function

$$
\widehat{U}(v, \theta)=V\left(\widetilde{r}_{\mathrm{h}}(v), \theta ; \mu, G_{0}\right)=\sum_{\ell \in \mathbb{Z}} \widehat{U}^{[\ell]}(v) e^{i \ell \theta},
$$

where $V$ is defined in (11) and $\widetilde{r}_{\mathrm{h}}$ in (10), are

$$
\widehat{U}^{[\ell]}(v)=\sum_{j \geq \max \left\{\delta_{0}(\ell),-\ell\right\}} c_{j} c_{j+\ell} \frac{\mu(1-\mu)^{2 j+\ell}+(-1)^{\ell}(1-\mu) \mu^{2 j+\ell}}{G_{0}^{4 j+2 \ell} \widetilde{r}_{\mathrm{h}}^{2 j+\ell+1}(v)},
$$

where $c_{j}=\left(\begin{array}{c}-1 / 2 \\ j\end{array}\right), \delta_{0}(0)=1$ and $\delta_{0}(\ell)=0$ for $\ell \neq 0$.

Proof. We use the identity

$$
\begin{aligned}
(1+A \cos \theta)^{-1 / 2} & =\alpha^{-1 / 2}\left(1+\beta e^{i \theta}\right)^{-1 / 2}\left(1+\beta e^{-i \theta}\right)^{-1 / 2} \\
& =\alpha^{-1 / 2} \sum_{j \geq 0} \sum_{k \geq 0} c_{j} c_{k} \beta^{j+k} e^{i(k-j) \theta} \\
& =\sum_{\ell \in \mathbb{Z}} e^{i \ell \theta} \sum_{\substack{k-j=\ell \\
j, k \geq 0}} c_{j} c_{k} \beta^{j+k} \\
& =\alpha^{-1 / 2} \sum_{\ell \in \mathbb{Z}} e^{i \ell \theta} \sum_{j \geq \max \{0,-\ell\}} c_{j} c_{j+\ell} \beta^{2 j+\ell},
\end{aligned}
$$


where $\alpha=A /(2 \beta)$ and $\beta=\left(1-\sqrt{1-A^{2}}\right) / A$, and

$$
\begin{aligned}
\frac{1-\mu}{\sqrt{G_{0}^{4} \widetilde{r}_{\mathrm{h}}^{2}-2 \mu G_{0}^{2} \widetilde{r}_{\mathrm{h}} \cos \theta+\mu^{2}}} & =\frac{1-\mu}{G_{0}^{2} \widetilde{r}_{\mathrm{h}}}\left(1-\frac{\mu}{G_{0}^{2} \widetilde{r}_{\mathrm{h}}} e^{i \theta}\right)^{-1 / 2}\left(1-\frac{\mu}{G_{0}^{2} \widetilde{\mathrm{h}}_{\mathrm{h}}} e^{-i \theta}\right)^{-1 / 2} \\
\frac{\mu}{\sqrt{G_{0}^{4} \widetilde{r}_{\mathrm{h}}^{2}+2(1-\mu) G_{0}^{2} \widetilde{r}_{\mathrm{h}} \cos \theta+(1-\mu)^{2}}} & =\frac{\mu}{G_{0}^{2} \widetilde{r}_{\mathrm{h}}}\left(1+\frac{1-\mu}{G_{0}^{2} \widetilde{r}_{\mathrm{h}}} e^{i \theta}\right)^{-1 / 2}\left(1+\frac{1-\mu}{G_{0}^{2} \widetilde{r}_{\mathrm{h}}} e^{-i \theta}\right)^{-1 / 2}
\end{aligned}
$$

to obtain

$$
\begin{aligned}
\frac{1}{G_{0}^{2}} V\left(\widetilde{r}_{\mathrm{h}}(v), \theta\right)= & \frac{1-\mu}{\sqrt{G_{0}^{4} \widetilde{r}_{\mathrm{h}}^{2}-2 \mu G_{0}^{2} \widetilde{r}_{\mathrm{h}} \cos \theta+\mu^{2}}}+\frac{\mu}{\sqrt{G_{0}^{4} \widetilde{r}_{\mathrm{h}}^{2}+2(1-\mu) G_{0}^{2} \widetilde{r}_{\mathrm{h}} \cos \theta+(1-\mu)^{2}}} \\
& -\frac{1}{G_{0}^{2} \widetilde{r}_{\mathrm{h}}(v)} \\
= & \frac{1-\mu}{G_{0}^{2} \widetilde{r}_{\mathrm{h}}(v)} \sum_{\ell \in \mathbb{Z}} e^{i \ell \theta} \sum_{j \geq \max \{0,-\ell\}} c_{j} c_{j+\ell} \frac{(-\mu)^{2 j+\ell}}{G_{0}^{4 j+2 \ell} \widetilde{r}_{\mathrm{h}}^{2 j+\ell}(v)} \\
& +\frac{\mu}{G_{0}^{2} \widetilde{r}_{\mathrm{h}}(v)} \sum_{\ell \in \mathbb{Z}} e^{i \ell \theta} \sum_{j \geq \max \{0,-\ell\}} c_{j} c_{j+\ell} \frac{(1-\mu)^{2 j+\ell}}{G_{0}^{4 j+2 \ell} \widetilde{r}_{\mathrm{h}}^{j+\ell}(v)}-\frac{1}{G_{0}^{2} \widetilde{r}_{\mathrm{h}}(v)} \\
= & \sum_{\ell \in \mathbb{Z}} e^{i \ell \theta} \sum_{j \geq \max \{0,-\ell\}} c_{j} c_{j+\ell} \frac{(-1)^{\ell}(1-\mu) \mu^{2 j+\ell}+\mu(1-\mu)^{2 j+\ell}}{G_{0}^{4 j+2 \ell+2} \widetilde{r}_{\mathrm{h}}^{2 j+\ell+1}(v)}-\frac{1}{G_{0}^{2} \widetilde{r}_{\mathrm{h}}(v)} .
\end{aligned}
$$

Now we prove Proposition 3.1

Proof Proposition [3.1. First, we observe that the Poincaré function $L$ in (28) can be written as

$$
L\left(v, \xi ; \mu, G_{0}\right)=\int_{-\infty}^{+\infty} V\left(\widetilde{r}_{\mathrm{h}}(t), \xi+G_{0}^{3} v-G_{0}^{3} t+\widetilde{\alpha}_{\mathrm{h}}(t) ; \mu, G_{0}\right) d t .
$$

Now, using that

$$
V\left(\widetilde{r}_{\mathrm{h}}(t), \xi+G_{0}^{3} v-G_{0}^{3} t+\widetilde{\alpha}_{\mathrm{h}}(t) ; \mu, G_{0}\right)=\sum_{\ell \in \mathbb{Z}} \widehat{U}^{[\ell]}(t) e^{i \ell \widetilde{\alpha}_{\mathrm{h}}(t)} e^{-i \ell G_{0}^{3} t} e^{i \ell\left(\xi+G_{0}^{3} v\right)}
$$

and therefore

$$
L\left(v, \xi ; \mu, G_{0}\right)=\sum L^{[\ell]} e^{i \ell\left(\xi+G_{0}^{3} v\right)}
$$

with

$$
L^{[\ell]}=\int_{-\infty}^{+\infty} \widehat{U}^{[\ell]}(t) e^{i \ell \widetilde{\alpha}_{\mathrm{h}}(t)} e^{-i \ell G_{0}^{3} t} d t
$$

to compute $L^{[\ell]}$ we use the expansions in Lemma A.1 obtaining, when $\ell \neq 0$

$$
L^{[\ell]}=\sum_{j \geq 0}\left(\begin{array}{c}
-\frac{1}{2} \\
j
\end{array}\right)\left(\begin{array}{c}
-\frac{1}{2} \\
\ell+j
\end{array}\right) \frac{\mu(1-\mu)^{2 j+\ell}+(-1)^{\ell}(1-\mu) \mu^{2 j+\ell}}{G_{0}^{4 j+2 \ell}} \mathcal{I}(\ell, j)
$$

with

$$
\mathcal{I}(\ell, j)=\int_{-\infty}^{+\infty} \frac{e^{i \ell \widetilde{\alpha}_{\mathrm{h}}(t)}}{\widetilde{r}_{\mathrm{h}}^{2 j+\ell+1}(t)} e^{-i \ell G_{0}^{3} t} .
$$

To compute $\mathcal{I}(\ell, j)$ we use the change of variables $v=\frac{1}{2}\left(\frac{1}{3} \tau^{3}+\tau\right)$ given in Lemma 4.1 obtaining

$$
\mathcal{I}(\ell, j)=(-1)^{\ell} 2^{2 j+\ell} \int_{-\infty}^{+\infty} \frac{e^{-i \ell \frac{G_{0}^{3}}{2}\left(\tau+\frac{\tau^{3}}{3}\right)}}{(\tau-i)^{2 j}(\tau+i)^{2 j+2 \ell}} d \tau:=(-1)^{\ell} 2^{2 j+\ell} I(-\ell, j, j+\ell),
$$


where we have introduced the notation

$$
I(\ell, m, n)=\int_{-\infty}^{+\infty} \frac{e^{i \ell \frac{G_{0}^{3}}{2}\left(\tau+\frac{\tau^{3}}{3}\right)}}{(\tau-i)^{2 m}(\tau+i)^{2 n}} d \tau
$$

to write the Poincaré function Fourier coefficients as

$$
L^{[\ell]}=\sum_{j \geq 0}\left(\begin{array}{c}
-\frac{1}{2} \\
j
\end{array}\right)\left(\begin{array}{c}
-\frac{1}{2} \\
\ell+j
\end{array}\right) \frac{\mu(1-\mu)^{2 j+\ell}+(-1)^{\ell}(1-\mu) \mu^{2 j+\ell}}{G_{0}^{4 j+2 \ell}}(-1)^{\ell} 2^{2 j+\ell} I(-\ell, j, j+\ell) .
$$

The first observation is that

$$
I(-\ell, n, m)=I(\ell, m, n)=\overline{I(\ell, m, n)} .
$$

Therefore all the Fourier coefficients are real and we just need to compute them for $\ell>0$ :

$$
L^{[\ell]}=\sum_{j \geq 0}\left(\begin{array}{c}
-\frac{1}{2} \\
j
\end{array}\right)\left(\begin{array}{c}
-\frac{1}{2} \\
\ell+j
\end{array}\right) \frac{\mu(1-\mu)^{2 j+\ell}+(-1)^{\ell}(1-\mu) \mu^{2 j+\ell}}{G_{0}^{4 j+2 \ell}}(-1)^{\ell} 2^{2 j+\ell} I(\ell, j+\ell, j)
$$

and then

$$
L(\xi, v)=2 \sum_{\ell \in \mathbb{N}} L^{[\ell]} \cos \ell\left(\xi+G_{0}^{3} v\right) .
$$

To compute the integrals $I(\ell, m, n)$ for $\ell>0$, one uses the method in Erd56] (see also [LS80a, MP94]) changing the path of integration to a suitable complex path $\operatorname{Re}\left(\tau+\frac{\tau^{3}}{3}\right)=0$ up to a neighborhood of the singularity $\tau=i$.

Using that $\tau+\frac{\tau^{3}}{3}=\frac{2}{3} i+\mathcal{O}\left((\tau-i)^{2}\right)$, to bound the integrals (see [DKdlRS12]) it is enough to reach a neighborhood of the singularity $\tau=i$ of order $\mathcal{O}\left(G_{0}^{-3 / 2}\right)$, obtaining that there exists a constant $K>0$ such that, for any $\ell \in \mathbb{Z}$ and $m, n \geq 1$ :

$$
|I(\ell, m, n)| \leq K G_{0}^{3 m-3 / 2} e^{-\frac{G_{0}^{3}}{3} \ell},
$$

and therefore

$$
\left|L^{[\ell]}\right| \leq\left(K G_{0}\right)^{\ell-3 / 2} e^{-\frac{G_{0}^{3}}{3} \ell} .
$$

To obtain the dominant terms of the function $L$, which correspond to $\ell=1,2$, we can use the results in DKdlRS12, (see also MP94]) to obtain

$$
\begin{aligned}
I(1,2,1) & =\frac{1}{6} \sqrt{\frac{\pi}{2}} G_{0}^{\frac{9}{2}} e^{-\frac{G_{0}^{3}}{3}}\left(1+\mathcal{O}\left(G_{0}^{-3 / 2}\right)\right) \\
I(2,2,0) & =2 \sqrt{\pi} G_{0}^{\frac{9}{2}} e^{-2 \frac{G_{0}^{3}}{3}}\left(1+\mathcal{O}\left(G_{0}^{-3 / 2}\right)\right) .
\end{aligned}
$$

Thus,

$$
\begin{aligned}
& L^{[1]}=-\frac{\mu(1-\mu)^{3}-(1-\mu) \mu^{3}}{4} \sqrt{\frac{\pi}{2}} G_{0}^{-\frac{3}{2}} e^{-\frac{G_{0}^{3}}{3}}\left(1+\mathcal{O}\left(G_{0}^{-3 / 2}\right)\right) \\
& L^{[2]}=2 \mu(1-\mu) \sqrt{\pi} G_{0}^{\frac{1}{2}} e^{-2 \frac{G_{0}^{3}}{3}}\left(1+\mathcal{O}\left(G_{0}^{-3 / 2}\right)\right) .
\end{aligned}
$$

\section{Acknowledgements}

The authors acknowledge useful discussions with V. Kaloshin and A. Gorodetski. They have been partially supported by the Spanish MCyT/FEDER grant MTM2009-06973 and the Catalan SGR grant 2009SGR859. M. G. and P. M warmly thank the Institute for Advanced Study for their hospitality, stimulating atmosphere and support. During his stay in the Institute for Advanced Study, M. G. was also partially supported by the NSF grant DMS-0635607. 


\section{References}

[AKN88] V.I. Arnold, V.V. Kozlov, and A.I. Neishtadt. Dynamical Systems III, volume 3 of Encyclopaedia Math. Sci. Springer, Berlin, 1988.

[BF04] I. Baldomá and E. Fontich. Exponentially small splitting of invariant manifolds of parabolic points. Mem. Amer. Math. Soc., 167(792):x-83, 2004.

[BFGS11] I. Baldomá, E. Fontich, M. Guàrdia, and T. M. Seara. Exponentially small splitting of separatrices beyond melnikov analysis: rigorous results. Preprint, 2011.

[DKdlRS12] A. Delshams, V. Kaloshin, A. de la Rosa, and T. Seara. Parabolic orbits in the restricted three body problem. Preprint, 2012.

[DS92] A. Delshams and T. M. Seara. An asymptotic expression for the splitting of separatrices of the rapidly forced pendulum. Comm. Math. Phys., 150(3):433-463, 1992.

[DS97] A. Delshams and T.M. Seara. Splitting of separatrices in Hamiltonian systems with one and a half degrees of freedom. Math. Phys. Electron. J., 3:Paper 4, 40 pp. (electronic), 1997.

[Erd56] A. Erdélyi. Asymptotic expansions. Dover Publications Inc., New York, 1956.

[Gel97] V. G. Gelfreich. Melnikov method and exponentially small splitting of separatrices. Phys. $D, 101(3-4): 227-248,1997$.

[Gel00] V. G. Gelfreich. Separatrix splitting for a high-frequency perturbation of the pendulum. Russ. J. Math. Phys., 7(1):48-71, 2000.

[GK10a] J. Galante and V Kaloshin. Destruction of invariant curves using the ordering condition. Preprint, available at http://www.terpconnect.umd.edu/ vkaloshi, 2010.

[GK10b] J. Galante and V Kaloshin. The method of spreading cumulative twist and its application to the restricted circular planar three body problem. Preprint, available at http://www.terpconnect.umd.edu/ vkaloshi, 2010.

[GK11] J. Galante and V. Kaloshin. Destruction of invariant curves in the restricted circular planar three-body problem by using comparison of action. Duke Math. J., 159(2):275-327, 2011.

[GK12] A. Gorodetski and V Kaloshin. Hausdorff dimension of oscillatory motions for restricted three body problems. Preprint, available at http://www.terpconnect.umd.edu/ vkaloshi, 2012.

[GOS10] M. Guardia, C. Olivé, and T. Seara. Exponentially small splitting for the pendulum: a classical problem revisited. Journal of Nonlinear Science, 20(5):595-685, 2010.

[Gua12] M. Guardia. Splitting of separatrices in the resonances of nearly integrable hamiltonian systems of one and a half degrees of freedom. Preprint available at http://arxiv.org/abs/1107.6042, to appear in Discrete and Continous Dynamical Systems A, 2012.

[HMS88] P. Holmes, J. Marsden, and J. Scheurle. Exponentially small splittings of separatrices with applications to KAM theory and degenerate bifurcations. In Hamiltonian dynamical systems, volume 81 of Contemp. Math. 1988.

[LMS03] P. Lochak, J.-P. Marco, and D. Sauzin. On the splitting of invariant manifolds in multidimensional near-integrable Hamiltonian systems. Mem. Amer. Math. Soc., 163(775):viii+145, 2003.

[LS80a] J. Llibre and C. Simó. Oscillatory solutions in the planar restricted three-body problem. Math. Ann., 248(2):153-184, 1980.

[LS80b] J. Llibre and C. Simó. Some homoclinic phenomena in the three-body problem. J. Differential Equations, 37(3):444-465, 1980. 
[McG73] R. McGehee. A stable manifold theorem for degenerate fixed points with applications to celestial mechanics. J. Differential Equations, 14:70-88, 1973.

[Mel63] V. K. Melnikov. On the stability of the center for time periodic perturbations. Trans. Moscow Math. Soc., 12:1-57, 1963.

[Mos73] J. Moser. Stable and random motions in dynamical systems. Princeton University Press, Princeton, N. J., 1973. With special emphasis on celestial mechanics, Hermann Weyl Lectures, the Institute for Advanced Study, Princeton, N. J, Annals of Mathematics Studies, No. 77.

[MP94] R. Martínez and C. Pinyol. Parabolic orbits in the elliptic restricted three body problem. J. Differential Equations, 111(2):299-339, 1994.

[Ner84] A. I. Nershtadt. The separation of motions in systems with rapidly rotating phase. Prikl. Mat. Mekh., 48(2):197-204, 1984.

[Poi90] H. Poincaré. Sur le problème des trois corps et les équations de la dynamique. Acta Mathematica, 13:1-270, 1890.

[Sau01] D. Sauzin. A new method for measuring the splitting of invariant manifolds. Ann. Sci. École Norm. Sup. (4), 34, 2001.

[Sit60] K. Sitnikov. The existence of oscillatory motions in the three-body problems. Soviet Physics. Dokl., 5:647-650, 1960 .

[Tre97] D. Treschev. Separatrix splitting for a pendulum with rapidly oscillating suspension point. Russ. J. Math. Phys., 5(1):63-98, 1997.

[Xia92] Z. Xia. Mel'nikov method and transversal homoclinic points in the restricted three-body problem. J. Differential Equations, 96(1):170-184, 1992. 